\title{
Free Communications
}

\section{FC1-81 Adipose Tissue and Obesity}

\section{Serum levels of pigment epithelium-derived factor (PEDF) are positively associated with acanthosis nigricans in obese adolescents Julia Galhardo; Linda P Hunt; Julian Shield \\ University of Bristol, School of Clinical Sciences, Bristol, United Kingdom}

Background: Circulating PEDF (Serpin F1) is up-regulated during adipogenesis, contributing to obesity induced insulin resistance. Furthermore, PEDF is abundant in stage I melanosomes and has been reported to increase pigment granules and the appearance of mature melanosomes in retinal pigment epithelium.

Objective: As acanthosis nigricans (AN) is a well recognised clinical marker of insulin resistance, we hypothesized that increased PEDF might be associated with the generation of AN.

Methods: AN, anthropometric measurements, circulating PEDF (ELISA) and metabolic profiles were assessed in 28 obese adolescents in a hospitalbased obesity clinic.

Results: Subjects with AN ( $\mathrm{n}=10)$ exhibited greater plasma levels of PEDF than those without (geometric mean 23.55 (range 15.2-40.2) vs 9.01 (range 5.5-18.7) $\mathrm{pg} / \mathrm{mL} ; \mathrm{p}<0.001$ ). Although log PEDF was positively correlated with $\log$ HOMA-IR $(r=0.779, p<0.001)$ as previously reported, for the same degree of insulin resistance, those with AN exhibited a 2.1-fold [95\% CI 2.02.3] higher level of PEDF.

Conclusions: Whilst AN is undoubtedly associated with insulin resistance, its appearance is not ubiquitous in patients at any given level of HOMA-IR. The higher levels of PEDF in those with AN comparative to those without with similar levels of resistance suggest that PEDF might be an important association in the generation of acanthosis nigricans.

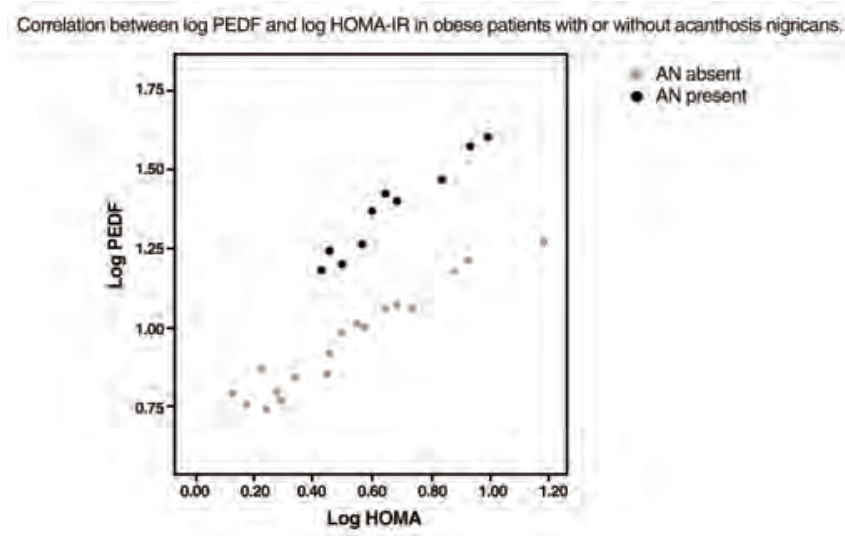

FC1-82 Adipose Tissue and Obesity

New key players in adipogenesis from GWAS?

Relevance of genes found associated with

obesity in Genome Wide Association studies in human adipocytes

Falk Bernhard; Kathrin Landgraf; Petra Büttner; Daniela Friebe;

Wieland Kiess; Antje Koerner

University of Leipzig, University Hospital for Children \& Adolescents,

Leipzig, Germany

Background: Recent Genome Wide Association studies (GWAs) identified a variety of new genes associated with obesity but their relevance in adipose tissue development has not been addressed so far.

Objective and hypotheses: The aim of our study was to characterize the respective genes in adipocytes by expression- and functional analyses in vitro. Methods: We selected eight genes (BDNF, MAF, MTCH2, NEGR1, NPC1, $P T E R, S H 2 B 1, T M E M 18$ ) from six GWAs and assessed their expression in metabolic, endocrine, neural and other tissues. We analysed the expression profile of each gene during adipocyte differentiation of human SGBS cells and determined the effect of metabolic factors, as insulin, dexamethasone, IGF-1 and isoproterenol on gene expression in both, adipocytes and preadipocytes. To investitigate a role of the genes during adipogenesis we used an siRNA-mediated knock down approach.

Results: All of the candidate genes were basally expressed in adipose tissue. MAF, MTCH2, and NEGR1 were slightly upregulated during SGBS adipocyte differentiation whereas $B D N F$ was downregulated to $30 \%$ compared to an undifferentiated control sample. There was no regulation of NPC1, PTER, SH2B1 and TMEM18 during adipogenesis. Furthermore, in adipocytes NEGR1 is upregulated by dexamethasone $(3.217 \pm 0.62$ fold, $p=0.0117)$ and downregulated by insulin ( $58 \%$ of control, $p=0.0085)$ and IGF-1 $(63.1 \%$, $p<0.0001)$. BDNF is significantly downregulated by stimulation with insulin $(59.6 \%$ of control, $p<0.0001)$, dexamethasone $(66.6 \%, p=0.0012)$ and IGF-1 (49.5\%, $p<0.0001)$ in adipocytes. Expression of MAF was inhibited by dexamethasone in both, preadipocytes $(35.1 \%$ of control, $p=0.0048)$ and adipocytes (30.0\%, $p=0.0010)$. Knock down of BDNF, MTCH2, NEGR1 and TMEM18 resulted in a significantly reduced triglyceride accumulation during adipogenesis whereas knock down of MAF, NPC1, PTER, and SH2B1 had no effect on adipocyte differentiation.

Conclusions: The results of expression studies and knock down analysis support a role of BDNF, MTCH2, NEGR1 and TMEM18 as potential new regulators of adipogenesis.

\section{FC1-83 Adipose Tissue and Obesity}

Targeted deletion of adipocytes by apoptosis leads to adipose tissue recruitment of

\section{alternatively activated M2-macrophages \\ Pamela Fischer-Posovszky'; Qiong Wang'; Ingrid Asterholm²; Joseph Rutkowski'; Martin Wabitsch ${ }^{1}$; Philipp Scherer ${ }^{2}$ \\ 'Ulm University, Division of Pediatric Endocrinology and Diabetes, \\ Ulm, Germany; 'University of Texas Southwestern Medical Center, \\ Touchstone Diabetes Center, Dallas, United States}

Background: Obesity is associated with an infiltration of macrophages into adipose tissue. The excessive accumulation of fat causes a phenotypic switch of macrophages from an alternatively activated M2-like phenotype towards a pro-inflammatory M1 phenotype. Macrophages are generally responsible for clearing old, pathological and dead cells from tissues.

Objective: In this study we investigate the role of fat cell apoptosis in adipose tissue macrophage accumulation.

Methods: We took advantage of the FAT-ATTAC (apoptosis through targeted activation of caspase-8) mouse model. Apoptosis of adipocytes was induced by administration of a FK1012 analog leading to the dimerization of a membrane-bound, adipocyte-specific caspase 8-FKBP fusion protein.

Results: After 2 weeks of dimerizer treatment we detected markedly reduced levels of adipose tissue and a robust down-regulation of circulating adipokines. qPCR and immunohistochemistry on epididymal and inguinal fat depots revealed an increase of the macrophage markers F4/80 and CD11c. Using polychromatic flow cytometry we detected an up-regulation of the alternatively activated M2 macrophage markers, CD206 and CD301, on F4/80 positive macrophages. 
Conclusions: Apoptosis of adipocytes caused an accumulation of alternatively activated, anti-inflammatory M2 macrophages. We conclude that apoptosis of adipocytes is a healthy process contributing to normal tissue homeostasis without causing a pro-inflammatory milieu. Other and/or additional factors are responsible for switching towards a M1 pro-inflammatory macrophage phenotype observed in obesity.

\section{FC1-84 Adipose Tissue and Obesity \\ TRAIL (TNF related apoptosis inducing ligand) affects adipogenic differentiation and adipocyte metabolism \\ Michaela Keuper'; Ingrid Asterholm²; Philipp Scherer² Anna-Maria Wolf ${ }^{3}$; Uwe Knippschild ${ }^{3}$; Martin Wabitsch ${ }^{1}$; Pamela Fischer-Posovszky \\ ${ }^{1}$ Ulm University, Division of Pediatric Endocrinology and Diabetes, UIm, Germany; ${ }^{2}$ UT Southwestern Medical Center, Touchstone Diabetes Center, Dallas, United States; ${ }^{3}$ Ulm University, Department of Visceral and Transplantation Surgery, UIm, Germany}

Background: TNF-alpha and other members of the tumor necrosis factor family affect adipose tissue metabolism and contribute to the obesity-related inflammation of adipose tissue.

Objective: Here, we sought to identify the effects of TRAIL (TNF related apoptosis inducing ligand) on human fat cell biology.

Methods: Human SGBS preadipocytes and adipocytes were used for studying adipogenic differentiation and adipocyte metabolism. Expression of TRAIL and its receptors was studied in human and murine adipose tissue. Results: TRAIL (10 ng/ml) inhibited adipogenic differentiation as shown by inhibited triglyceride accumulation ( $50 \%$ vs control) and decreased adipocyte marker gene expression. In human adipocytes TRAIL inhibited insulinstimulated glucose uptake ( $26 \%$ vs control) and de novo lipogenesis ( 25 $\%$ vs control) paralleled by decreased expression of lipogenic genes and an inhibition of Akt phosphorylation. Basal lipolysis was stimulated by $\sim 44 \%$. Furthermore, TRAIL up-regulated expression of inflammatory cytokines and down-regulated the expression of leptin and adiponectin. In human adipose tissue the expression of TRAIL receptors correlated positively with BMI. Overnight fasting decreased TRAIL and its receptor expression in murine adipose tissue.

Conclusions: We show here for the first time that TRAIL has significant effects on human adipocyte biology. Expression of TRAIL and its receptors is regulated by chronic and acute energy imbalance in human and murine adipose tissue. We conclude that TRAIL functions not only as an apoptosisinducing ligand, but additionally modulates metabolic functions. These findings have important implications for cancer research since agonistic TRAIL receptor antibodies are currently tested as anti-cancer agents.

\section{FC1-85 Adipose Tissue and Obesity \\ Myocardial mass and left ventricular geometry in obese adolescents}

Tetyana Chaychenko; Ganna Senatorova

Kharkiv National Medical University, Pediatrics 1 with Neonatology,

Kharkiv, Ukraine

Background: Studies implicate visceral adiposity and insulin resistance, with detrimental effects described on early surrogate markers of risk such as endothelial and myocardial function. To further evaluate this risk, we have assessed the contribution of body mass together with insulin resistance upon cardiac structure in obese and overweight adolescents.

Objective and hypotheses: We hypothesise that increasing body mass through insulin resistance contributes to detrimental changes in cardiac structure and function in adolescent children.

Methods: 82 obese (BMI $>97$ th age-sex percentile) and 20 overweight (BMI 85-97th age-sex percentile) adolescents (10-18 years) were studied using HOMA calculations of insulin resistance and tissue doppler echocardiography. Subjects were age and sex matched and grouped according to BMI: +12SD (Gp 1); 2-3SD (Gp 2) and >3SD (Gp 3). Cardiac structure was assessed using measures of Left Ventricular Mass (LVM), LVM indexed (LVMI), Posterior Ventricular Thickness (PVT), Septal Wall thickness (SWT) and LV function assessed using percent ejection fraction (\%EF).

Results: There was significantly increased $(\mathrm{p}<0.01)$ myocardial hypertro- phy as revealed by PVT and SWT in obese versus overweight adolescents. Inter-group analysis revealed significant increases in LVMI $(\mathrm{p}<0.05)$ with increasing weight (Gp1, 28,313+1,23 g/m2,7; Gp2 42,611+1,21 g/m2,7; Gp3 $49,62+2,88 \mathrm{~g} / \mathrm{m} 2,7)$. An eccentric LV hypertrophy was seen in all groups together with progressively increasing SWT $(\mathrm{P}<0.01$ between $\mathrm{Gp} 1$ and $\mathrm{Gp} 3$ ). There was a significant inter-group decline in $\% \mathrm{EF}(\mathrm{p}<0.01$ between $\mathrm{Gp} 1$ and $\mathrm{Gp3}$ ), although measures remained within the normal range. There were direct relationships between HOMA and LVMI $(r=0.45)$ plus SWT $(r=0.83)$ and an indirect relationship with \%EF ( $\mathrm{r}=0.42)$.

Conclusions: This study reveals detrimental changes in cardiac geometry and decreasing LV function associated with increasing body mass in otherwise healthy adolescents. The relationship between insulin resistance and detrimental structural changes suggests a causative role.

\section{FC1-86 Adipose Tissue and Obesity \\ Longitudinal multicenter analysis of glucose metabolism development in obese children \\ Antje Körner ; Susanna Wiegand; Andreas Hungele ${ }^{3}$; Sabine Tuschy ${ }^{4}$; Klaus Peter Otto ${ }^{5}$; Dagmar I'Allemand ${ }^{6}$; Kurt Widhalm"; Wieland Kiess ${ }^{1}$, Reinhard Holl \\ 1 University of Leipzig, Pediatric Research Center, Leipzig, Germany; 'University of Berlin, Charité Children's Hospital, Berlin, Germany; ${ }^{3}$ University of UIm, Institute of Epidemiology, Ulm, Germany; ${ }^{4}$ Sana Kliniken Berlin-Lichtenberg, Abt. Adipositas, Berlin, Germany; ${ }^{5}$ Kath. Kinderkrankenhaus Wilhelmstift, Tagesklinik, Hamburg, Germany; ${ }^{6}$ Ostscheizer Kinderspital, Pediatric Endocrinology, St. Gallen, Switzerland; ${ }^{7}$ Medical University Vienna, Pediatric Endocrinology, Vienna, Austria}

Rationale: Although there is ample evidence of impaired glucose metabolism in obese children from single cohorts, data are lacking on the progression of glucose metabolism in obese children. We performed a large-scale multicentric longitudinal analysis on glucose metabolism development in obese children.

Methods: Between years 2000 and 2010, 179758 patient visits were documented in the APV registry by 162 central European centers specialized in

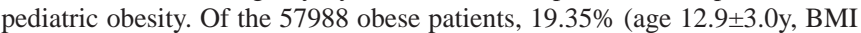
SDS $2.59 \pm 0.58, n=10364$ ) were evaluated by oGTT and $1.88 \%$ had at least two oGTTs. Patients were stratified for glucose metabolism pathology with impaired fasting glucose (IFG), impaired glucose tolerance (IGT) and type 2 diabetes (T2D).

Results: A total of $12.6 \%$ of the children presented with pathology in glucose metabolism (5.99\% IFG, 5.51\% IGT, 1.07\% T2D). Children with more overt pathology (IGT and T2D) were significantly older, more (visceral) obese, and the percentage of girls was higher. Multivariate analysis identified pubertal group and BMI as significant predictors for impaired glucose metabolism. For the 929 patients with follow-up oGTT (observation interval $1.48 \pm 1.32 \mathrm{y}$ ), we observed a slight though significant reduction in BMI SDS of 0.094 \pm 0.42 . Along with this, mean levels of metabolic parameters improved. While $18.8 \%$ of these patients showed abnormalities in glucose metabolism at baseline, the percentage decreased to $13.7 \%$ at follow-up. Of the children with IGT initially, more than 50\% converted to normal glucose tolerance. Overall, the oGTT result deteriorated in only $8.5 \%$, while $13.8 \%$ improved. The change in BMI SDS predicted improvement in oGTT as well as AUC BG, while sex, age, observation time, age and even BMI SDS at baseline did not have significant impact in multivariate analyses.

Conclusions: We provide evidence for significant improvement of oGTT parameters of obese patients treated in specialized treatment centers, even if reduction in BMI is mild. 


\section{FC2-87 Adrenal}

Mutations in GPX1 and NNT, encoding antioxidant defence genes, cause familial glucocorticoid deficiency (FGD)

Louise Metherell'; Eirini Meimaridou'; Julia Kowalczyk'; Leonardo Guasti'; Nicholas Mann²; Ritwik Banerjee ${ }^{3}$; Nurcin Saka4; Philippe Touraine ${ }^{5}$; Claire Hughes ${ }^{1}$; Xingen Lei ${ }^{6}$; Peter King ${ }^{1}$; Adrian Clark ${ }^{1}$

'William Harvey Research Institute, Centre for Endocrinology, London, United Kingdom; ${ }^{2}$ Royal Berkshire Hospital, Department of Paediatrics, Reading, United Kingdom; ${ }^{3}$ Luton \& Dunstable Hospital, Paediatric Endocrinology, Luton, United Kingdom; ${ }^{4}$ Istanbul University, Child Health Institute, Istanbul, Turkey; ${ }^{5}$ Université Pierre et Marie Curie, Groupe Hospitalier Pitié Salpêtrière, Paris, United Kingdom; ${ }^{6}$ Cornell University, Department of Animal Science, New York, United States

Background: Inherited ACTH resistance syndromes are rare and include triple A syndrome and FGD. Clinically, FGD is characterized only by ACTH resistance, while triple A exhibits additional clinical features including neurological impairments. Mutations in MC2R, MRAP or STAR, cause half of FGD cases and mutations in AAAS encoding ALADIN are responsible for most Triple A cases.

Objective: To find further genetic cause(s) of FGD.

Methods: SNP array genotyping of FGD patients mapped disease loci to chromosome 3p26-24 and 5p13-q12.

Results: Targeted exome sequencing of 5p13-q12 in one patient identified a homozygous mutation, p.Ala533Val, in nicotinamide nucleotide transhydrogenase (NNT), a protein involved in antioxidant defence. Further mutations, p.Tyr201PhefsX2 and p.Leu977Pro, were discovered by conventional sequencing in two other families. The antioxidant gene glutathione peroxidase 1 (GPX1) was then identified within the 3p26-24 region and sequencing detected a homozygous mutation, p.Arg130-Leu133del, in one individual. RT-PCR revealed that NNT and GPX1 are both highly expressed in human adrenals. C57BL6J mice (a natural Nnt mutant) showed some evidence of adrenal hyperplasia and their baseline and ACTH-stimulated corticosterone levels were significantly lower than wild-type mice. Interestingly an autopsy on a 5 month old sibling of the GPX1 patient showed loss of the zona fasciculata but preservation of the zona glomerulosa.

Conclusions: Under most physiological conditions, NNT uses energy from the mitochondrial proton gradient to produce the high concentrations of NADPH necessary for ROS detoxification by enzymes such as GPX1. Previous studies showed that ROS-mediated disruption of Leydig cell mitochondria inhibits STAR function and steroidogenesis, and recent work implicates oxidative stress in the pathogenesis of triple A. Our results suggest that NNT and GPX1 are critical for ROS detoxification in adrenocortical cells, with their loss leading to defective oxidative stress responses, an impairment of steroidogenesis and adrenal insensitivity to ACTH.

\section{FC2-88 Adrenal}

Increased activation of the 'backdoor pathway' in patients with 21 hydroxylase deficiency: evidence from urinary steroid metabolomics Clemens Kamrath' ; Ze'ev Hochberg'; Michaela Hartmann'; Stefan A. Wudy

1 Justus-Liebig-University, Department of Pediatrics, Giessen, Germany; ${ }^{2}$ Rambam Medical Center, and Rappaport Family Faculty of Medicine and Research Institute, Technion-Israel Institute of Technology, Division of Pediatric Endocrinology, Meyer Children's Hospital, Haifa, Israel

Background: In the fetal gonads of the tammar wallaby, 17-hydroxyprogesterone (17-OHP) can be converted to $5 \alpha$-pregnane-3 $3,17 \alpha$-diol-20-one (pdiol) by sequential activities of $5 \alpha$-reductase and $3 \alpha$-hydroxysteroid dehydrogenase. Pdiol has high affinity to human CYP17 and is an excellent substrate for the 17,20 lyase activity, and therefore a key intermediate metabolite of the ,backdoor pathway' providing an alternative route to dihydrotestosterone (DHT).

Objective and hypotheses: The objective of this study was to obtain evidence for the presence of the ,backdoor pathway' in 21-hydroxylase deficiency (21-OHD).

Methods: We analysed urinary steroid hormone profiles using gas chroma- tography-mass spectrometry analysis of 143 untreated patients (age range: 1 day to 25.4 years; 51 males) with 21-OHD and compared the results with 143 age- and sex-matched control subjects. The activity of the ,backdoor pathway' was calculated using the metabolite ratio of the urinary excretion of pdiol to the sum of the urinary excretion of the DHEA metabolites (DHEAms: DHEA, $16 \alpha$-hydroxy-DHEA, 5-androsten-3 $\beta, 17 \alpha$-diol, 5 -androsten- $3 \beta, 17 \beta$ diol and androstentriol-16 $\alpha$ ) and androstenedione (AD) metabolites (ADms: 11-hydroxyandrosterone and etiocholanolone) resulting from the $\Delta 5$ and $\Delta 4$ ,frontdoor pathways': pdiol x 100/ (DHEAms + ADms).

Results: There was an increased ratio of the urinary pdiol excretion to the sum of the excretion of the DHEA and AD metabolites in 21-OHD subjects (median $(25$ th; 75 th percentiles): $4.1(1.4 ; 11.7)$ as compared to controls $(0.6$ (0.2; 1.3), $\mathrm{P}<0.0001)$.

Conclusions: The elevated ratio indicates a higher activity of the $17-\mathrm{OHP} \rightarrow$ pdiol $\rightarrow$ DHT , backdoor pathway' compared to the ,frontdoor' $\Delta 5$ and $\Delta 4$ pathways via DHEA and AD in $21-$ OHD subjects.

\section{FC2-89 Adrenal}

\section{Identification and characterization of the promoter region for cytochrome P450 oxidoreductase gene}

\section{Tsutomu Ogata; Shun Soneda; Maki Fukami}

National Research Institute for Child Health and Development, Department of Molecular Endocrinology, Tokyo, Japan

Background: Cytochrome P450 oxidoreductase (POR) deficiency (PORD) is a rare autosomal recessive disorder characterized by skeletal dysplasia, adrenal dysfunction, 46,XY and 46,XX disorders of sex development, and maternal virilization during pregnancy. Although $P O R$ gene is known to consist of at least a single non-coding exon 1 and coding exons 2-6, its promoter region remain to be identified.

Patients: We studied three Japanese patients with PORD (cases 1-3) in whom DNA sequencing analysis showed heterozygosity for the R457H mutation and RT-PCR sequencing analysis revealed transcription failure of the apparently normal alleles.

Molecular studies: Genome DNA analysis identified heterozygous microdeletions in cases 1-3; a 2,487 bp deletion involving untranslated POR exon 1 in case 1, and the same 49,604 bp deletion involving exons 1-2 in cases 2 and 3 . In silico analysis: Three putative Sp1 binding sites (sites A-C from the transcription start site) were identified within the 2,487 bp deleted sequence common to cases 1-3.

Methylation analysis: Bisulfite methods using leukocyte genomic DNA demonstrated virtually unmethylated patterns of sites A-C.

In vitro analysis: $\mathrm{Sp} 1$ protein was shown to bind to the three putative Sp1 binding sites A-C by EMSA. Relative luciferase activity was markedly increased ( 120 times) by using a reporter construct with a $\sim 300$ bp fragment harboring sites $\mathrm{A}-\mathrm{C}$, whereas deletions of site $\mathrm{A}$, site $\mathrm{B}$, site $\mathrm{C}$, and sites $\mathrm{A}+\mathrm{B}+\mathrm{C}$ decreased luciferase activities by $\sim 80 \%, \sim 15 \%, \sim 5 \%$, and nearly $100 \%$, respectively.

Conclusion: The present study implies that the region encompassing the three unmethylated $\mathrm{Sp} 1$ binding sites functions as the component of promoter for POR. Since Sp1 binding sites are often identified in the promoter regions of housekeeping genes, this would also be consistent with ubiquitous expression of $P O R$. Further studies will permit to understand the promoter apparatus for $P O R$. 


\section{FC2-90 Adrenal \\ Aldosterone synthase (CYP11B2) deficiency: clinical, hormonal, molecular genetic and in vitro functional studies of a large cohort (31 patients) \\ Florence Roucher-Boulez'; Ingrid Plotton'; Claire-Lise Gay; Catherine Raynaud-Ravni'; Rita Menassa'; Yves Morel' \\ ${ }^{1}$ Molecular Endocrinology and Rare Diseases, CBPE, Hospices Civils de Lyon, Bron-Lyon, France; ${ }^{2}$ Pediatric Endocrinology, Hopital Femme Mere Enfant, Hospices Civils de Lyon, Bron-Lyon, France; ${ }^{3}$ Pediatrics, General hospital, Saint Chamond, France}

Background: Aldosterone synthase deficiency, due to mutations in the CYP11B2 gene, inherited as an autosomal recessive manner, generally results in a salt-wasting (SW) crisis in infancy. CYP11B2 catalyzes the last three steps of aldosterone biosynthesis: $11 \beta$-hydroxylation converting 11-deoxycorticosterone to corticosterone, 18-hydroxylation leading to 18-hydroxycorticosterone (18-OHB) and finally 18-oxidation. Aldosterone deficiencies' classification, based on plasma 18OHB, distinguishes two types. Nevertheless, no good correlation has been observed with the functional studies probably due to normal CYP11B1 activities (11ß-/18-hydroxylations).

Objective and hypotheses: For a better understanding of this disorder, we report clinical, hormonal and molecular studies of our cohort: 27 families, 31 patients.

Methods: All the patients presented with a failure to thrive during the neonatal period followed by a biological or clinical SW occuring before three months of life. The diagnosis was suspected in presence of hyponatremia, high renin contrasting with low aldosterone and normal ACTH and confirmed by CYP11B2 gene sequencing.

Results: All the patients were homozygous or compound heterozygous for CYP11B2 mutations. The different mutations found were 8 nonsense/frameshift, 15 missense and 2 in frame deletion mutations. In vitro studies are in progress for 11 new mutations using a nonsense mutant as a control of the absence of the three CYP11B2 activities. Preliminary results show three patterns: 1) a complete defect for five mutations; 2) exclusively a residual 11-hydroxylation activity for three mutations; 3) normal 11-hydroxylase activity with weak residual C18 activities for one mutation.

Conclusions: In conlusion, this disorder seems more frequent as reported. The correlation between the nature and the severity of the enzymatic defects and the phenotype were in progress. Moreover, these studies should help to elucidate the structure-function involved in the three steps of aldosterone biosynthesis. Thanks to all clinicians for providing clinical data and DNA.

\section{FC2-91 Adrenal \\ Mutation analysis of CYP11B2 gene in 12 patients with primary hypoaldosteronism. One novel mutation (IVS8+1G>A) reported Christina Merakou; Maria Dracopoulou; Amalia Sertedaki; Antonis Voutetakis; Christina Kanaka-Gantenbein; George Chrousos; Catherine Dacou-Voutetakis \\ University of Athens, Medical School, First department of Pediatrics, Division of Endocrinology, Metabolism and Diabetes, Athens, Greece}

Background: Primary hypoaldosteronism or Aldosterone synthase deficiency is usually manifested in infancy with failure to thrive, hyponatremia, hyperkalemia, normal cortisol and ACTH, high renin and low aldosterone values. The underlying molecular defects in the majority of cases are inactivating mutations in the CYP11B2 gene.

Objective and hypotheses: To define the underlying molecular defect in our cohort of patients presenting with primary hypoaldosteronism.

Methods: Twelve patients with phenotype compatible with primary hypoaldosteronism confirmed by appropriate hormonal evaluation were studied. In these patients and their parents, DNA analysis of the CYP11B2 gene was carried out. CYP11B2 gene was selectively amplified against the highly homologous CYP11B1 gene and all 9 exons and exon-intron boundaries were directly sequenced.

Results: Eight patients were found homozygotes for the p.T185I mutation in exon 3 of the CYP11B2 gene. One patient was compound heterozygote for the mutations p.T185I and p.T318M (exon 5). One patient was compound heterozygote for the mutation p.T185I and the double mutation p.I263N $+p$. V386A (exons 4 and 7). One patient was compound heterozygote for the mu- tations p.R174-R175del (exon 3) and the double mutation p.I263N+p.V386A. One patient was compound heterozygote for the mutation p.T185I and a novel mutation IVS8+1G>A (intron 8).

Conclusions: The predominant defect in our patients with primary hypoaldosteronism, of variable geographic origin, is the p.T185I mutation in exon 3 of the CYP11B2 gene (detected in 19 out of 24 alleles studied). A novel mutation, the IVS8+1G>A, was detected in one patient of Albanian origin. This mutation disrupts the donor splice site of intron 8 of the CYP11B2 gene, leading to the production of an altered protein. Phenotype to genotype concordance was encountered in all our patients.

\section{FC2-92 Adrenal \\ Novel mutations in the Steroidogenic Acute Regulatory Protein (StAR) in 46, XY cases with adrenal insufficiency and complete sex reversal \\ Fatih Gurbuz'; 'L. Damla Kotan²; Fatih Temiz'; A. Kemal Topaloglu' Bilgin Yuksel ${ }^{1}$ \\ Cukurova University Faculty of Medicine, Pediatric Endocrinology, Adana, Turkey; ${ }^{2}$ Cukurova University Institute of Sciences, Biotechnology, Adana, Turkey}

Background: The steroidogenic acute regulatory protein (StAR) has been shown to be essential for steroidogenesis by mediating cholesterol transfer into mitochondria. Inactivating StAR mutations cause the typical clinical picture of congenital lipoid adrenal hyperplasia.

Objective and hypotheses: We aimed to identify causative mutations in cases presenting with adrenal failure during early infancy.

Methods: Consecutive cases with adrenal failure during early infancy were studied. The coding regions of the StAR gene (uc003xkv.1) is PCR-amplified and automatedly sequenced.

Results: A compound heterozygous state consisting of p.W147R and p.E169K was detected in a patient.

A homozygous state of the latter variant p.E169K was identified in another patient.

A previously reported mutation of p.A218V was demonstrated in yet another family. Functional studies of the new mutations are ongoing.

Conclusions: The novel mutations of p.W147R and p.E169K as well as the recurring mutation of p.A218V cause early infancy adrenal insufficiency and complete sex reversal in the $46, \mathrm{XY}$ cases.

\section{FC3-93 Pituitary}

\section{Heparan sulfate 60-sulfotransferase 1 (HS6ST1), a gene involved in extracellular sugar modifications, is mutated in patients with idiopathic hypogonadotrophic hypogonadism (IHH)}

Janne Tornberg'; Gerasimos Sykiotis'; Kimberly Keefe; Lacey Plummer ; Xuan Hoang ${ }^{3}$; Janet Halß; Richard Quinton ${ }^{4}$; Stephanie Seminara ${ }^{3}$; Virginia Hughes ${ }^{3}$; Guy Van Vliet'; Stan Van Uum ${ }^{6}$; William F. Crowley ; Wilson Chung7; Pei-San Tsai Hiroko Habuchi; ${ }^{8}$ Koji Kimata ${ }^{8}$; Hannes Bülow ${ }^{9}$; Nelly Pitteloud ${ }^{10}$ ${ }^{1}$ Albert Einstein College of Medicine, Genetics, New York, United States; ${ }^{2}$ University of Patras Medical School, Endocrinology, Patras, Greece; ${ }^{3}$ Massachusetts General Hospital, Reproductive Endocrine Unit, Boston, United States; ${ }^{4}$ Royal Victoria Infirmary, Endocrinology, Newcastle-Upon-Tyne, United Kingdom; ${ }^{5}$ Centre Hospitalier Univeritaire Sainte-Justine, Pediatrics, Montreal, Canada; ${ }^{6}$ Aichi Medical University, Genetics, Aichi, Japan; ${ }^{7}$ University of Colorado, Department of Integrative Physiology, Boulder, United States; 8 University of Western Ontario, Medicine, London, Canada; ${ }^{9}$ Albert Einstein College of Medicine, Genetics \& Neuroscience, New York, United States; ${ }^{10}$ Centre Hospitalier Universitaire Vaudois, Endocrinology, Diabetes, \& Metabolism, Lausanne, Switzerland

Background: Neuronal development is the result of a multitude of neural migrations, which require extensive cell-cell communication. These processes are modulated by extracellular matrix components such as heparan sulfate (HS) polysaccharides. HS is molecularly extraordinarily complex due to nonrandom modifications of the sugar moieties, including sulfations in specific 
positions. We hypothesize that rare variants in (HS6ST1) could underlie cases of idiopathic hypogonadotropic hypogonadism (IHH) - a condition characterized by incomplete or absent puberty and infertility as a result of defects in gonadotropin-releasing hormone $(\mathrm{GnRH})$ neuron development or function.

Objective and hypotheses: To screen IHH patients and healthy normal controls for variants in HS6ST1, characterize the in-vitro functionality of rare variants, and elucidate the in vivo mechanisms using a C. elegans assay for genetic interactions with IHH genes (kal-1, fibroblast growth factor [FGF], and its receptor FGFR).

Methods: IHH probands (271 males, 67 females) and healthy controls $(n=500)$ were studied. Heparan sulfate was used to assess mutant sulfotransferase activity in vitro. The cDNAs were cloned, constructs were injected to create transgenic animals, and neuroanatomy scored.

Results: We identified 1 homozygous and 4 heterozygous HS6ST1 mutations among $7 \mathrm{IHH}$ probands that were not seen in controls. The IHH probands exhibit a broad spectrum of $\mathrm{GnRH}$ deficiency with variable olfactory phenotypes (from normal to anosmia). All mutants were loss-of-function in vitro. Genetic experiments in C. elegans revealed that HS cell-specifically regulates neural branching in vivo in concert with other $\mathrm{IHH}$-associated genes including kal-1, FGF, and FGFR.

Conclusions: We report novel loss-of-function mutations in HS6ST1 accounting for $2 \%$ of $\mathrm{IHH}$ cases. All mutations display reduced activity in vitro and in vivo suggesting that HS6ST1 and the complex modifications of extracellular sugars are critical for signaling during development of the nervous system in humans.

\section{FC3-94 Pituitary}

\section{Mutations in NR5A1, PIN1 associated with idiopathic hypogonadotropic hypogonadism}

Sicui Hu

Huazhong University of Science and Technology, Pediatrics, Wuhan, China

Background: Idiopathic hypogonadotropic hypogonadism (iHH) is characterized by delayed or absent sexual development associated with inappropriately subnormal serum concentrations of LH (luteinizing hormone) and FSH (follicle stimulating hormone)and sex steroid levels in the absence of anatomical or functional abnormalities of the hypothalamic - pituitary-gonadal axis. IHH gene defects including GPR54, FGF8, FGFR1,NELF, KAL1, GnRH, GnRHR, GnRH1, PROKR2, CHD7, LHRH, PROK2, TAC3, TACR3, SF1,DAX-1 and so on. We hypothesized that NR5A1 and pin1 mutations could be identified in IHH.

Methods: Specimens of peripheral blood were collected from 50 sporadic Idiopathic hypogonadotropic hypogonadism and 100 normal control subjects, who have normal karyotype and there was no abnormal is discovered in magnetic resonance imaging (MRI) scan of the pituitary, meanwhile exhausting other endocrine diseases. The genome DNA was extracted. The $2-7$ exons and splice-sites of NR5A1 gene and the $1-4$ exons and Splice-sites of PIN1 gene were amplified with PCR, and the products of the $50 \mathrm{IHH}$ and 100 normal control subjects without IHH were sequenced. Results of DNA sequenced were analyzed and mutation site were determined. The clinical data of these families were collected and the correlation between genotype and phenotype was analyzed.

Results: NR5A1 and Pin 1 mutations were found in 7 out of 50 cases. These seven individuals presented with severely low serum concentration of testosterone or estradiol and gonadotropin. To date, adrenal insufficiency has not occurred in any of the patients.

Conclusions: NR5A1 or Pin1 mutations should be considered in male or female IHH patiens with normal karyotype and without insufficiency of adrenal function.

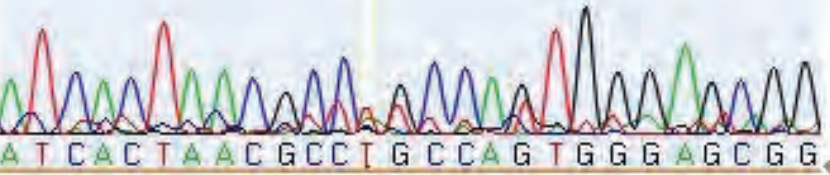

CG GGC CAB T G G T T CAAG C T G GA
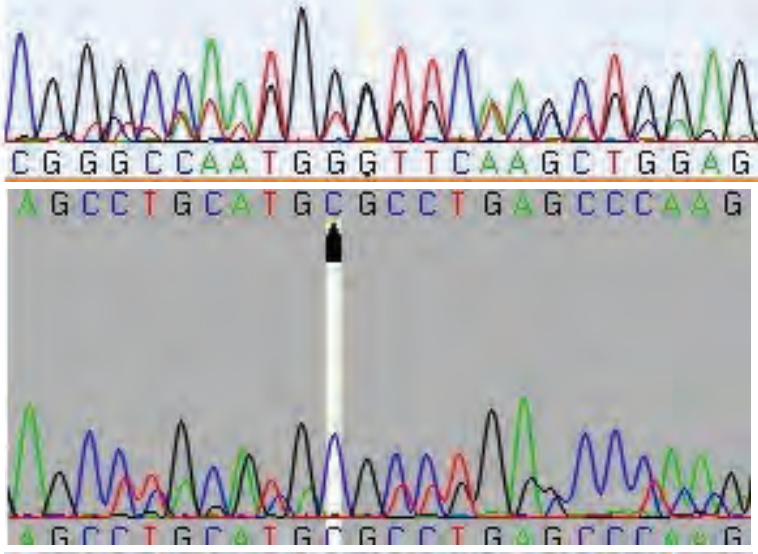

$\triangle G \subset C T G C A T G C G \subset C T G \triangle G C \subset C A B G$

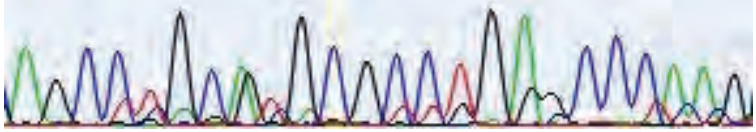

$A G C C T G C A T G C G C C T G A G C \subset C A A G$

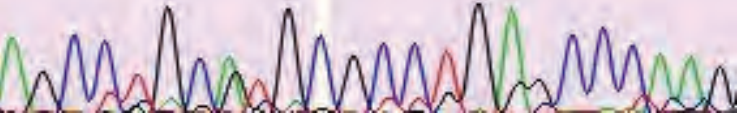

C CACACCGCTGGCTCTGCATGGT TCATT T T C 1

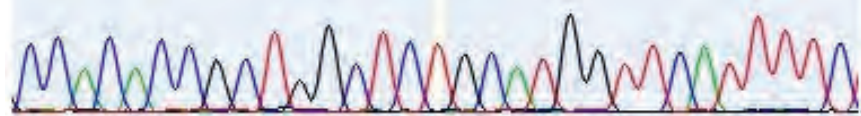

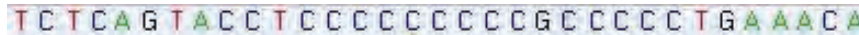
1

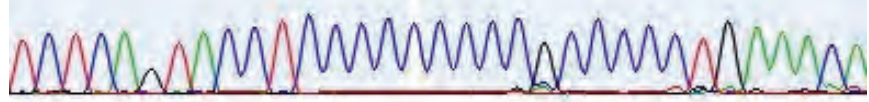




\section{FC3-95 Pituitary \\ Comparative functional analysis of two fibroblast growth factor receptor 1 (FGFR1) mutations effecting the same residue ( $R 254 W$ and R254Q) in hypogonadotropic hypogonadism (HH)}

Vasiliki Koika ${ }^{1}$; Helen Valavani ${ }^{2}$; Petros Varnavas ${ }^{1}$; Yisrael Sidis ${ }^{3}$; Lacey Plummer"; Richard Quinton ${ }^{5}$; Christina Kanaka-Gantenbein², Nelly Pitteloud ${ }^{3}$; Neoklis A. Georgopoulos'

Catherine Dacou-Voutetakis²; Amalia Sertedaki

'University of Patras, Department of Obstetrics and Gynecology, Division of Reproductive Endocrinology, Patras, Greece; ${ }^{2}$ University of Athens, Medical School, First Department of Pediatrics, Division of Endocrinology, Diabetes and Metabolism, Athens, Greece; 'University of Lausanne, School of Biology and Medicine, Department of Physiology and Service of Endocrinology, Lausanne, Switzerland; ${ }^{4}$ Massachusettes General Hospital, Reproductive Endocrine Unit, Massachusettes, United States; ${ }^{5}$ University of Newcastle-upon-Tyne, Institute of Human Genetics, Newcastle-upon-Tyne, United Kingdom

Background: FGFR1 mutations have been identified in about $10 \%$ of patients with Kallman Syndrome and in $7 \%$ of patients with normosmic $\mathrm{HH}$ (nIHH). To date, only a few mutations in FGFR1 have been characterized in-vitro using structural and functional studies.

Objective and hypotheses: To define the in-vitro functionality of two FGFR1 mutations (R254W and R254Q) resulting from different amino acid substitutions in the same residue. To relate the in-vitro findings to structural predictions and patient phenotypes.

Methods: Patient 1 presented with a severe type of nIHH. His mother had menarche at $17 y r s$, irregular menses, normal fertility and late onset anosmia. Patient 2, was a sporadic, severe case of KS. We compared expression levels and signalling activity of mutants with a wild type (WT) receptor in-vitro. Receptor expression levels were assessed by Western blot. Expression at the cell surface was measured by radiolabelled antibody binding assay. Signalling activity was determined by a FGF2/FGFR1-dependent transcription reporter assay.

Results: A novel FGFR1 mutation, R254W was identified in patient 1 and his mother. Patient 2 harboured a R254Q mutation. Both mutants, R254W and $\mathrm{R} 254 \mathrm{Q}$, display diminished protein expression levels in-vitro (40 and $30 \%$ reduction relative to WT, respectively), while protein maturation was unaffected. Cell surface expression levels of the mutant receptors were also significantly reduced $(30 \% \mathrm{p}<0.001$ and $15 \% \mathrm{p}<0.05$, respectively). Accordingly, maximal receptor signalling capacity was reduced by $45 \%(\mathrm{p}<0.001)$ and $15 \%(\mathrm{p}<0.05)$, respectively, compared to WT.

Conclusions: The R254W and R254Q are loss-of-function mutants as demonstrated by their reduced overall and cell surface expression levels. These data suggest a deleterious effect on receptor folding and stability rather than an impact on ligand binding. Tryptophan substitution at R254 is more disruptive than the more conserved glycine substitution. No clear relation between the phenotypic presentation and severity of loss-of-function of mutants invitro was found.

\section{FC3-96 Pituitary \\ A novel missense sequence variant in CHD7 associated with hypopituitarism \\ Mark McCabe ${ }^{1}$; Evelien Gevers ${ }^{2}$; Louise Gregory ; Joanna Baker ${ }^{3}$; Dragona Josifov'; Mehul Dattani \\ ${ }^{1}$ Institute of Child Health, Department of Endocrinology, Clinical and Molecular Genetics Unit, London, United Kingdom; ${ }^{2}$ Great Ormond Street Hospital for Children, Department of Metabolic Endocrinology and Gastroenterology, London, United Kingdom; ${ }^{3}$ Kent and Canterbury Hospital, Department of Pediatrics, Canterbury, United Kingdom; ${ }^{4}$ Guy's Hospital, Department of Genetics, London, United Kingdom}

Background: Pathologic mutations in the chromatin remodelling protein CHD7 have been implicated in CHARGE syndrome. CHARGE is associated with hypogonadotrophic hypogonadism but not usually with other pituitary hormone deficiencies. We present a 7 year old boy with severe short stature (-4.9 SDS), Tetralogy of Fallot, bilateral severe sensori-neural hearing loss, abnormally shaped ears, a squint, trigonocephaly, a small occipital meningocele, micropenis and severe developmental delay. Investigations revealed mild secondary hypothyroidism and severe GHD (GH peak on provocation $0.7 \mu \mathrm{g} / \mathrm{L}$, undetectable IGF1 and IGFBP3), with no evidence of other hormone deficiencies. MRI showed a small anterior pituitary and a posterior pituitary at the tuber cinereum.

Hypothesis: CHD7 is expressed in both Rathke's pouch and the diencephalon during human embryogenesis and is found in complex with proteins involved in ES cell differentiation and pituitary development. Given the proband's phenotype, we hypothesized that the he may bear a mutation in CHD7.

Methods: The coding region of $C H D 7$ was screened by PCR and direct sequencing.

Results: A heterozygous sequence variant was identified in exon 4 of CHD7 (c. $2194 \mathrm{C}>\mathrm{G}$, p. P732A) at a highly conserved residue in the index case and the mother who is short and has had hypothyroidism since the age of 11, consistent with the known variable penetrance of $C H D 7$ mutations. The variant was not found in 100 other CHARGE patients. Additionally no CHD7 mutations were identified in 100 individuals with congenital hypopituitarism, thus not presently implicating CHD7 in isolated forms of the condition.

Conclusion: We have identified a CHD7 sequence variant in a child with features of CHARGE syndrome and hypopituitarism associated with an ectopic posterior pituitary, the first such association reported to our knowledge. Further investigation of the mother and genetic analysis of the child are needed to assess the relation between the sequence variant and the phenotype observed.

\section{FC3-97 Pituitary \\ Considerable frequency of novel GLI2 missense mutations in patients with hypopituitarism without holoprosencephaly}

Marcela Franca; Alexander Jorge; Aline Otto; Luciani Carvalho;

Berenice B Mendonca; Ivo J. P. Arnhold

Faculdade de Medicina da Universidade de Sao Paulo, Unidade de

Endocrinologia do Desenvolvimento, LIM-42, Sao Paulo, Brazil

Background: GLI2 is a zinc finger transcription factor downstream of Sonic Hedgehog signaling, acting in the early phase of pituitary and diencephalon embryogenesis. Recently, nonsense GLI2 mutations were first described in patients with hypopituitarism without holoprosencephaly. Data from these patients included ectopic posterior pituitary lobe and variable: pituitary hormone deficiencies, polydactyly and midline facial defects.

Objective and hypotheses: To study GLI2 in a cohort of 180 patients with isolated GH deficiency (IGHD) or combined pituitary hormone deficiency (CPHD).

Methods: The GLI2 coding region was amplified by PCR using intronic primers followed by automatic sequencing.

Results: In 21 patients, we identified heterozygous missense GLI2 mutations: p.A203T, p.P253S, p.A268V, p.R473H, p.A780V, p.F830L, p.R933H, p.G947D, p.V1117L, p.G1197D, p.M1241I/P1485A are novel, p.A268V, p.G1197D, p. M1444I/L1445F and M1352V/D1520N had been previously published. These mutations are located in GLI2 activator and repressor domains. According to in silico analysis p.P253S, p.A268V, p.P1485A and p.D1520N mutations are probably damaging; p.F830L, p.M1444I and p.L1445F are possibly damaging and the others are probably benign. All patients had CPHD and MRI showing ectopic posterior lobe; except for two patients with p.G1197D and p.M1444I/L1445F mutations who had IGHD and topic posterior pituitary lobe. No patient had polydactyly, midline or cerebral defects.

Conclusions: We detected 16 different heterozygous missense GLI2 mutations, with a significant frequency $(12 \%)$ in our cohort. Patients had IGHD or CPHD and ectopic posterior pituitary lobe (the majority) without holoprosencephaly. Data from this study suggest an important role for GLI2 mutations in the etiology of congenital hypopituitarism, especially when associated with an ectopic posterior pituitary lobe. 


\section{FC3-98 Pituitary}

Mutations in Sonic Hedgehog (SHH) signaling pathway genes in congenital hypopituitarism patients with or without midline cerebral defects

Sabrina Soares Paulo'; Fábio Luiz Fernandes-Rosa';

Carlos Eduardo Martinelli Jr'; Wendy Turatti Diniz;

Antônio Carlos Santos ${ }^{3}$; Ayrton Moreira²; Margaret Castro²;

Sonir R. Antoninit

${ }^{1}$ School of Medicine of Ribeirao Preto- University of Sao Paulo,

Pediatrics, Ribeirao Preto-SP, Brazil; ${ }^{2}$ School of Medicine of Ribeirao

Preto- University of Sao Paulo, Internal Medicine, Ribeirao Preto-SP,

Brazil; ${ }^{3}$ School of Medicine of Ribeirao Preto- University of Sao Paulo,

Radiology-Internal Medicine, Ribeirao Preto-SP, Brazil

Background: The SHH signaling pathway regulates early central nervous system (CNS) and pituitary development. Disruption of this pathway can result in midline cerebral defects (MCD) and/or congenital hypopituitarism (CH).

Objective and hypotheses: To investigate the presence of mutations in the SHH and GLI2 genes in patients with CH and/or MCD.

Methods: Clinical, biochemical, CNS magnetic resonance imaging and mutation screening were performed in 118 patients from a single University Hospital. Entire coding and boundary regions of the SHH and GLI2 genes were automated sequenced.

Results: Mean age at diagnosis was 5.8 years $(0.7-14.4)$ in septo-optic dysplasia patients (SOD; $n=32), 9$ years $(6.5-11.7)$ in patients with isolated anomalies of corpus callosum (ACC; $n=9$ ), and 10.7 years $(1.2-27)$ in $\mathrm{CH}$ patients without MCD $(n=62)$. Holoprosenchefaly (HPE) was antenatally diagnosed in most of patients $(n=15)$. Endocrine deficiencies (combined pituitary hormone deficiencies-CPHD with or without ADH deficiency or isolated GH deficiency-GHD) were found in $69 \%, 67 \%$, and $53 \%$ in SOD, ACC, and HPE patients, respectively. In the HPE patients group, a novel SHH mutation (p.Y175X) was found in a patient with alobar HPE, midline cleft lip, ocular hypotelorism and CPHD. No SHH mutations were found in the other groups. In the coding region of GLI2 gene we identified six missense heterozygous variants: p.V183M in one patient with GHD; p.V432M in one patient with CPHD; p.[M1444I $(+) \mathrm{L} 1445 \mathrm{~F}]$ in 3 patients with hypopituitarism, one of them presenting a solitary median maxillary central incisor (SMMCI); p.A268V in two patients with SOD without hypopituitarism; and p.L761F in one patient with SOD and CPHD. We also found 4 synonymous GLI2 variants (c.414G>A, c.642C > T, c.720C > T, c.2187C > T).

Conclusions: $\mathrm{SHH}$ mutation was found only in one patient with HPE. GLI2 mutations were found in patients with variable phenotype spectrum, including SOD with or without $\mathrm{CH}$, SMMCI and CPHD or isolated GHD without midline facial or cerebral defects.

\section{FC4-99 Bone and Mineral Metabolism}

\section{5-Hydroxyvitamin D3-1 a -Hydroxylase activity in the duodenum as a function of 25-Hydroxyvitamin D3 levels}

Aneta Gawlik'; Nimrod Rozen²; Aviva Dahan 3 ; Ron Shaul';

Yehuda Chowers ${ }^{5}$; Dov Tiosano ${ }^{6}$

${ }^{1}$ Medical University of Silesia, Department of Pediatric Endocrinology and Diabetes, Silesia, Poland; ${ }^{2}$ Aemeck Hospital, Bone and mineral research Division, Afula, Israel; ${ }^{3}$ Rambam Health Care Campus, Gastroenterology research Laboratory, Haifa, Israel; 'Meyer Children's Hospital, Pediatric Gastroenterology, Haifa, Israel; ${ }^{5}$ Rambam Health Care Campus, Department of Gastroenterology, Haifa, Israel; ${ }^{6}$ Meyer Children's Hospital, Pediatric Endocrinology, Haifa, Israel

Context: Vitamin D-deficiency (VDD) is associated with low 25OHvitamin $\mathrm{D}(25(\mathrm{OH}) \mathrm{D} 3)$ but high serum1,25(OH)2D3. Why then VDD causes reduced calcium absorption, secondary hyperparathyroidism and rickets? HYPOTHESIS: Calcium absorption requires intra-duodenal generation of 1,25(OH) 2D3 by duodenal $1 \alpha$-OHase, and its activity is $25(\mathrm{OH}) \mathrm{D} 3$ dependent.

Methods: Biopsy-derived duodenal explants from 14 individuals age 4-25 years were placed with the villous surface up on microporous membranes in Millicell-HA tissue culture insertsin $10 \%$ fetal calf serumand trypsin inhibitor. $25 \mathrm{OH}$ D3-1 1 hydroxylase activity was assessed by 60 minutes $1,25(\mathrm{OH}) 2 \mathrm{D} 3$ generation from 0-400 ng/mL added substrate 25(OH)D3.
Results: Basal generation of 1,25(OH)2D3 was $8.69+3.88 \mathrm{pg} / \mathrm{ml} / \mathrm{mg}$ protein/ hr. It increased dose dependently as a function of added 25(OH)D3 (10 - 400 $\mathrm{ng} / \mathrm{mL}$ ), reaching its max of $400 \mathrm{ng} / \mathrm{mL}$ at $246 \mathrm{ng} / \mathrm{mL} 25 \mathrm{OHD} 3$, with a $\mathrm{K} 0.5$ of $125 \mathrm{ng} / \mathrm{ml}$. To study the role of estrogen and PTH on duodenal $1 \alpha$-Ohase, explants were incubated with escalating concentrations of estradiol and PTH in the presence of $200 \mathrm{ng} / \mathrm{ml}$ 25OHD. Neither had any effect on $1 \alpha$-Ohase activity.

Conclusions: Here we show for the first time human duodenal 25OHD-1 $\alpha$ OHase activity and is PTH independency. Human calcium absorption requires $25 \mathrm{OHD}$ as a substrate and local duodenal generation of 1,25(OH)2D3 for intra- and paracrine activity. Hence, VDD cannot be treated or prevented with calcitriol and requires vitamin $\mathrm{D}$ supplementation.

\section{FC4-100 Bone and Mineral Metabolism}

\section{Treatment of children with hypophosphatasia (HPP) with ENB-0040: radiographic and DXA} outcomes after 6 months of therapy

Michael P. Whyte ${ }^{\prime}$; Katherine L. Madson ${ }^{1}$; Cheryl R. Greenberg';

William H. McAlister'; Deborah Wenkert'; Amy L. Reeves';

Karen E. Mack'; Lise Bourrier" ${ }^{4}$ Thomas D. Thacher';

Fan Zhang'; Rebecca L. Hulett ${ }^{3}$; Thomas E. Herman ${ }^{3}$;

Geetika Khanna3; Ali M. Skrinar ; Hal Landy ${ }^{7}$

${ }^{1}$ Shriners Hospital for Children, Center for Metabolic Bone Disease and Molecular Research, St. Louis, United States; ${ }^{2}$ University of Manitoba, Biochemistry and Medical Genetics, Winnipeg, Canada; ${ }^{3}$ Washington University School of Medicine, Mallinckrodt Institute of Radiology, St. Louis, United States; ${ }^{4}$ Manitoba Institute of Child Health, Clinica Research Unit, Winnipeg, Canada; ${ }^{5}$ Mayo Clinic, Family Medicine, Rochester, United States; ${ }^{6}$ Enobia Pharma, Clinical Research, Montreal, Canada; ${ }^{7}$ Enobia Pharma, Medical Affairs, Montreal, Canada

Background: HPP is the heritable rickets/osteomalacia due to inactivating mutation(s) in the gene that encodes the tissue non-specific isoenzyme of alkaline phosphatase (TNSALP). The skeletal spectrum ranges from stillbirth due to profound skeletal hypomineralization to acquired osteomalacia in adults.

ENB-0040 is a bone-targeted, recombinant TNSALP protein being studied in HPP.

Objective: Evaluate 6 mo of ENB-0040 treatment in HPP children (ages 5-12 yrs) assessed by metaphyseal changes using two radiographic scales and changes in bone mineral density(BMD).

Design/methods: A 6-mo, multi-center, open-label, historical control study of 13 children receiving ENB-0040 subcutaneously (2 or $3 \mathrm{mg} / \mathrm{kg} 3 \mathrm{X}$ wkly). The controls were untreated children with HPP of similar age and clinical characteristics whose $\mathrm{x}$-ray and DXA data were retrieved from a natural history database.

Two scales were used by central, "blinded” readers. A Radiographic Global Impression of Change (RGI-C) was developed to assess HPP rickets. In this 7 -point scale, -3 represents severe worsening and +3 complete healing of rickets. Pts with a mean score of $\geq+2$ were "responders".

The Rickets Severity Scale (RSS: J Trop Peds 46:132,'00) is a 10-point scale that scores nutritional metaphyseal changes in wrists and knees. The score progresses from zero (no rickets) to 10 points (severe rickets).

DXA (Hologic:QDR4500A) evaluated spine BMD at baseline and 6 mo.

Data were analyzed by intention-to-treat.

Results: One pt withdrew for elective surgery. Using the RGI-C scale, 9 of 13 pts $(69 \%)$ were responders vs. 2 of $17(12 \%)$ historical controls $(p=0.002)$ The mean change in RSS in treated pts was -1.808 vs. -0.588 in the historical controls $(\mathrm{p}=0.0013)$.

Lumbar spine mean BMD z-score at baseline was $-2.2 \pm 1.4$ vs. $-1.5 \pm 1.3$ at 6 mo ( $p=0.0063)$. The change in 6 mo for the treated pts was +0.7 as compared to a semi-annualized change in lumbar spine BMD z-score for the controls of +0.07 .

Conclusions: Six mo treatment with ENB-0040 significantly improved HPP as assessed by two different radiographic rickets scales and BMD assessed by DXA. 


\section{FC4-101 Bone and Mineral Metabolism}

A randomized controlled comparison of zolidronate to pamidronate effects upon bone mineralization and fracture incidence in children with severe osteogenesis imperfect

\section{Shadab Salehpour ${ }^{1}$; Saeed Tavakoli}

${ }^{1}$ Shaheed Beheshti University of Medical Sciences, Pediatric

Endocrinology, Tehran, Islamic Republic of Iran; ${ }^{2}$ Tehran University of Medical Sciences, Pediatric Orthopedics, Tehran, Islamic Republic of Iran

Background: Severe osteogenesis imperfect (OI) is a disorder characterized by osteopenia, frequent fractures, progressive deformities, loss of mobility, and chronic bone pain.

Objective: The main objective of this study was to compare the efficacy and safety of zolidronate with those of pamidronate on bone mineralization and fracture incidence in OI.

Methods: The study was performed at the outpatient pediatric endocrine clinic of a children hospital. 27 prepubertal children (2-11 years old) with severe OI divide randomly in two groups, treated with either zolidronate (13 patients) or pamidronate (14 patients) for 2 years. The main outcome measures were bone mineral density and fracture incidence. Secondarily, their effects on mobility and bone turnover markers (alkaline phosphatase and Ctelopeptide) were compared.

Results: Zolidronate and pamidronate had the same effects on improving the bone mineral density $(p<0.001)$ and reducing the fracture incidence $(p<0.001)$. Their effects on mobility and bone turnover markers were also similar.

Conclusions: The efficacy of zolidronate and pamidronate on improving bone mineralization and reducing fracture incidence and their safety in children with severe OI are the same.

\section{FC4-102 Bone and Mineral Metabolism}

Inhibition of osteoblast differentiation by a GTPase-deficient mutant of the extra-large Gas variant XLas: implications for the pathogenesis of fibrous dysplasia

Serap Turan'; ${ }^{1}$ Zun Liu²; Murat Bastepe 2

${ }^{1}$ Marmara University, Pediatric Endocrinology, Istanbul, Turkey;

${ }^{2}$ Massachusetts General Hospital, Harvard Medical School, Endocrine Unit, Boston, United States

Background: Fibrous dysplasia of bone is caused by postzygotic mutations within GNAS, which encodes the $\alpha$-subunit of the stimulatory G protein (Gas) and its variant XL $\alpha$ s. These mutations inhibit the GTPase activities of Gas and XL $\alpha$ s and cause constitutive cAMP generation. Constitutively active (CA) XL $\alpha$ s and Gos mutants are different regarding their subcellular localization. Objective and hypotheses: We asked whether basal cAMP generation induced by a GTPase deficient mutant $X L \alpha s-R 543 H(C A X L \alpha s)$ is different from that induced by the cognate mutant $G \alpha s-R 201 H(C A G \alpha s)$ and asked whether constitutive XL $\alpha$ s and Gas activities can have different effects on bone differentiation.

Methods: Transfected HEK293 cells were examined regarding basal cAMP levels and transfected MC3T3-E1 cells were examined regarding osteoblastic differentiation. Two days after transfection of MC3T3-E1 cells, osteoblastic differentiation was induced by addition of ascorbic acid (day 0), and the cells were grown for 5 days. Cells were analyzed at days 0,2 , and 5 by staining for alkaline phosphatase (ALP) activity and measuring the levels of ALP and transforming growth factor-beta1 (TGF $\beta 1)$ mRNA.

Results: HEK293 cells expressing CA XLas showed significantly higher accumulation of cAMP than those expressing CA Gas when incubated with a cAMP phosphodiesterase inhibitor. Control cells transfected with the empty vector differentiated into early osteoblasts by day 5, showing strong ALP staining and high levels of ALP and TGF $\beta 1$ mRNA. Cells transiently expressing CA Gas showed lower degree of ALP staining and modestly increased levels of ALP and TGF $\beta 1 \mathrm{mRNA}$, thus showing impaired osteoblastic differentiation. On the other hand, almost no indication of osteoblastic differentiation was observed in cells transiently expressing CA XL $\alpha$ s.

Conclusions: These results indicate that constitutively active XL $\alpha$ s is a robust inhibitor of osteoblast differentiation and can therefore contribute to the pathogenesis of fibrous dysplasia.

\section{FC4-103 Bone and Mineral Metabolism}

Early-onset osteoporosis associated with an unbalanced chromosome 4:20 translocation in multiple members of a pedigree

Esther Kinning' ${ }^{1}$; Vidya K Narayanan'² ${ }^{2}$ Kiran Adil Bhatti',

Catherine McWilliam³; Christopher I Adams"; Edward S. Tobias';

S. Faisal Ahmed²

${ }^{1}$ Royal Hospital for Sick Children (Yorkhill), Department of Clinical Genetics, Glasgow, United Kingdom; ${ }^{2}$ Royal Hospital for Sick Children (Yorkhill), Department of Child Health, Glasgow, United Kingdom; ${ }^{3}$ Aberdeen Royal Infirmary, Department of Clinical Genetics, Aberdeen, United Kingdom; ${ }^{4}$ Royal Hospital for Sick Children, Edinburgh, Scottish National Spine Deformity Centre, Edinburgh, United Kingdom

Background: Linkage studies have previously highlighted an association between osteoporosis and the terminal region of chromosome $4 \mathrm{q}$.

Objective and hypotheses: We report the case of a 16 year old girl who presented via the spinal surgeons with kyphosis, scoliosis and a history of frequent fractures following minimal trauma. Family history revealed a sister and a male first cousin with similar problems. Both girls had a ventricular septal defect and all three children had a similar unusual facial appearance, vesicoureteric reflux, sensori-neural hearing loss and developmental delay. The affected children did not have dentinogenesis imperfecta or physical features of a collagen abnormality and routine bone biochemistry was normal. Initial DXA in the index case showed a TB BMC for BA SDS of -0.2 and a LS BMC for BA SDS of -1.3 which improved to 0.4 and -0.5 respectively after 2 years treatment with oral risedronate. X-ray abnormalities included a thoracic kyphoscoliosis, loss of vertebral height and partial fusion of C5/6.

Methods and results: Chromosome analysis by karyotyping and FISH revealed an unbalanced translocation resulting in a $4 \mathrm{q}$ terminal deletion and a 20p terminal duplication in the three cases. The imbalance had arisen as a result of a balanced reciprocal translocation in the girls' father and the boy's mother, themselves siblings. A parent with the balanced translocation was shown to have normal BMD. aCGH showed an 8 megabase deletion of terminal chromosome 4q incorporating a region previously linked to low BMD and a 15 megabase duplication of terminal chromosome 20p. Amongst the 39 genes deleted on chromosome 4q, there are no known genes that are, to date, associated with primary childhood osteoporosis.

Conclusions: The cosegregation of the skeletal phenotype with the unbalanced translocation and the deleted region on chromosome $4 \mathrm{q}$ raises the possibility of a novel gene within the deleted region with a critical role in skeletal development.

\section{FC4-104 Bone and Mineral Metabolism \\ Decreased responsiveness to epinephrine in patients with acrodysostosis, multiple hormonal resistance and a recurrent mutation in PRKARIA}

Agnes Linglart'; Philippe Chanson²; Emmanuelle Motte'; Eric Clauser ${ }^{3}$; Pierre Bougnères ${ }^{1}$; Caroline Silve ${ }^{4}$

${ }^{1}$ Bicêtre Hospital, Paris-Sud, APHP, Pediatric Endocrinology and INSERM U986, Le Kremlin-Bicetre, France; ${ }^{2}$ Bicêtre Hospital, Paris-Sud, APHP, Service d'Endocrinologie et des Maladies de la Reproduction, Le Kremlin-Bicetre, France; ${ }^{3}$ Hôpital Européen Georges Pompidou (HEGP), APHP, U970, Centre de Recherche Cardiovasculaire, Paris, France; ${ }^{4}$ INSERM, INSERM U986, Paris, France

Background: Acrodysostosis is a rare disease characterized by features resembling Albright osteodystrophy, including obesity, that are found in pseudohypoparathyroidism Ia (PHP1a). Recently we identified a germline recurrent heterozygous mutation in the PRKARIA gene, coding for the regulatory subunit of the protein kinase (PKA) in 3 patients with acrodysostosis and multiple hormonal resistance. The mutation causes PRKAR1A gain-of-function, which represses constitutively the catalytic subunit and results in PKA insensitivity to cAMP, thereby explaining the phenotype and the similarities with PHP1a.

Objective and hypotheses: We documented in these patients resistance to $\mathrm{PTH}, \mathrm{TSH}, \mathrm{GHRH}$ and gonadotropins. We hypothesized that resistance to Epinephrine, which signals through Gsa and PKA, could contribute to the obesity seen in acrodysostosis as previously demonstrated in PHP1a. 
Methods: We investigated the effect of exogenous epinephrine infusion (35 $\mu \mathrm{gr} / 1 \mathrm{~h}$ ) on heart rate (HR), plasma glycerol, and free fatty acids (FFA) in 2 patients and 6 lean controls.

Results: Results are presented as a mean \pm SD of 3 repeated measures. HR was higher in patients with acrodysostosis $(74 \pm 4)$ than in controls $(60 \pm 3)$. However, HR in patients did not increase during epinephrine infusion (98 \pm $2 \%$ of basal HR), whereas in controls it increased significantly $(114 \pm 3 \%$, $\mathrm{p}=0.001)$. Patients glycerol $(80.0 \pm 14.1$ microM $)$ and FFA $(0.33 \pm 0.03 \mathrm{mM})$ were comparable to those of controls $(91.8 \pm 10.1$ and $0.52 \pm 0.07, p=0.52$ and 0.06, respectively for glycerol and FFA). However, epinephrine infusion induced a lower production of glycerol and FFA in patients $(91.2 \pm 4.8$ and $0.55 \pm 0.08$, respectively) than in controls (146.2 \pm 8.6 and $1.00 \pm 0.07$, respectively).

Conclusions: These results are consistent with reduced epinephrine responsiveness in the 2 patients with acrodysostosis and PRKAR1A mutation as observed in patients with PHP-Ia. The data indicate that the deficient cAMP signaling and end-organ resistance to adrenergic stimulation might contribute to the obesity seen in both diseases.

\section{FC5-105 Growth Hormone}

\section{Treatment with recombinant human growth hormone during childhood is associated with increased intraocular pressure}

Ilan Youngster ${ }^{1}$; Rony Rachmiel2; Orit Pinhas-Hamie/ ${ }^{3}$; Bistritzer Tzvi'; Nehama Zuckerman-Levin ${ }^{4}$; Liat de Vries ${ }^{5}$; Ori Eyalf; Larisa Nagolani" Marianna Rachmiel'

${ }^{1}$ Asaf Harofeh Medical Center, Division of Pediatrics, Zerifin, Israel; ${ }^{2}$ Tel-Aviv Sourasky Medical Center, Department of ophthalmology, Tel-Aviv, Israel; ${ }^{3}$ Safra Children's Hospital, Pediatric Endocrine and Diabetes Unit, Ramat-Gan, Israel; ${ }^{4}$ Clalit Health Services, Sharon-Shomron District, Kfar-Saba, Israel; ${ }^{5}$ Schneider Children's Medical Center, (6) The Jesse Z and Sara Lea Shafer Institute for Endocrinology and Diabetes, Petah Tiqwa, Israel; ' ${ }^{6}$ ana Children's Hospital, Pediatric Endocrinology and Diabetes Unit, Tel-Aviv, Israe

Background: Recombinant human growth hormone (rhGH) is widely used to treat various growth disorders in the pediatric population, with therapy often continuing for as long as 12 years. Thus, knowledge of the short- and longterm safety of this treatment is essential. An association between endogenous growth hormone levels and increased intraocular pressure (IOP) has previously been reported. Increased IOP may lead to asymptomatic progressive optic nerve damage and consequent visual loss. Hence, early diagnosis and treatment are crucial to ensure optimal visual outcome.

Objective: To evaluate the association between rhGH treatment and IOP in children.

Methods: The study group comprised of 55 children aged 5-18 years, treated for at least 12 months with rhGH. All underwent an ocular examination, including slit lamp assessment and tonometry. Their charts were reviewed for treatment duration, peak stimulated GH level prior to therapy, IGF1 level prior to treatment and at time of examination, and rhGH dosage. Results were compared to pediatric norms and to a matched group of children with similar growth disorders, examined prior to commencement of rhGH treatment.

Results: Mean age at examination was $11.5 \pm 3.3$ years. patients were treated for a mean of $37.6 \pm 23.3$ months and average rhGH dose was $0.04 \pm 0.01$ $\mathrm{mg} / \mathrm{kg} / \mathrm{d}$. Mean IOP was $15.8 \pm 2.3 \mathrm{mmHg}$ and $16.3 \pm 2.4 \mathrm{mmHg}$ in the left and right eye respectively. Values are significantly increased compared both to the mean IOP reported in the pediatric population in the literature $(\mathrm{p}<0.001)$, and to values recorded in the control group $(\mathrm{p}<0.001)$. The IOP was positively related to rhGH dose ( $\mathrm{r} 2=0.095 ; \mathrm{p}=0.03$ ), and to treatment duration ( $\mathrm{r} 2=$ 0.19; $\mathrm{p}=0.001$ ).

Conclusion: There is a significant increment in IOP among children treated with rhGH, associated with longer duration and higher doses. We suggest that routine monitoring of ocular pressure should be considered in GH treated children.

\section{FC5-106 Growth Hormone}

GH secretory pattern in non-obese children and adolescents with Prader-Willi syndrome

Graziano Grugni' ; Antonino Crinò2; Sara Pagani; Paola Travaglino3, Fabio Buzi4; Teresa De Toni5; Alba Pilotta4; Luigi Gargantini;;

Giovanni Battista Pozzan ${ }^{7}$; Giorgio Radetti ; Letizia Ragusa ${ }^{9}$; Mauro Bozzola ${ }^{3}$

${ }^{1}$ Italian Auxological Institute, Department of Auxology, Verbania, Italy; Bambino Gesù Children's Hospital, Unit of Autoimmune Endocrine

Diseases, Rome, Italy; ${ }^{3}$ University of Pavia, Department of Pediatrics Pavia, Italy; ${ }^{4}$ University of Brescia, Department of Pediatrics, Brescia, Italy; ${ }^{5}$ University of Genoa, Department of Pediatrics, Genoa, Italy; ${ }^{6}$ Civic Hospital, Department of Pediatrics, Teviglio (BG), Italy; ${ }^{7}$ University of Padua, Department of Pediatrics, Padua, Italy; ${ }^{8}$ Regional Hospital, Department of Pediatrics, Bolzano, Italy; ${ }^{9}$ Oasi Maria S.S. Hospital, Department of Pediatric Endocrinology, Troina (EN), Italy

Background: Short stature is a cardinal feature of Prader-Willi syndrome (PWS). Growth failure appears to be due to a lack of the pubertal growth spurt and to the presence of a GH/IGF-I deficiency. Nevertheless, the impaired GH secretory pattern has been related to the presence of obesity only.

Objective and hypotheses: To further clarify whether the suboptimal GH secretion in PWS is an artifact of the obesity, this study aimed to evaluate either the GH immunological activity and the GH bioactivity after a standard provocative test in a group of non-obese children and adolescents with PWS Methods: Twenty-three patients with genetically confirmed PWS, 16 males, aged $6.9 \pm 0.9$ years, were included in the study. The published Italian standards for sex- and age-specific Body Mass Index (BMI: $\left.\mathrm{w} / \mathrm{h}^{2}\right)$ percentiles were used for calculating standard deviation score (SDS). All PWS subjects were non-obese (BMI <2.0 SDS); BMI SDS ranged from -2.90 to 1.94 (mean \pm SE: $0.63 \pm 0.26)$. As a control group, we evaluated 32 healthy children, matched to PWS subjects for gender, age and BMI SDS (0.21 \pm 0.2$)$. All subjects underwent a standard arginine test for the determination of $\mathrm{GH}$ immunological activity [measured by immunofluorimetric assay (IFMA)] and GH bioactivity (evaluated by Nb2 cells). GH responses were evaluated either as mean peak values (GHp: $\mu \mathrm{g} / \mathrm{l}$ ) or as the area under the curve (AUC, $\mu \mathrm{g} / \mathrm{l} / \mathrm{h}$ ).

Results: GHp after pharmacological stimulation was significantly lower in PWS compared with controls when measured by IFMA $(6.06 \pm 1.23 \mu \mathrm{g} / \mathrm{l}$ vs $23.7 \pm 1.06 \mu \mathrm{g} / 1, \mathrm{p}<0.0001)$. Similarly, analysis of GH AUC confirmed that the PWS group differed significantly from the controls $(387.9 \pm 76.1 \mu \mathrm{g} / \mathrm{l} / \mathrm{h}$ vs $1498.1 \pm 56.2 \mu \mathrm{g} / \mathrm{l} / \mathrm{h}, \mathrm{p}<0.0001)$. Evaluation of $\mathrm{GHp}$ by $\mathrm{Nb} 2$ assay showed a lower GH bioactivity in PWS compared to healthy subjects $(6.87 \pm 0.55 \mu \mathrm{g} / \mathrm{l}$ vs $12.88 \pm 0.19 \mu \mathrm{g} / \mathrm{l}, \mathrm{p}<0.0001)$.

Conclusions: Our results are in agreement with the hypothesis that a complex derangement of hypothalamus-pituitary axis occurred in PWS, indicating that GH insufficiency in children with PWS is not an artefact of obesity.

\section{FC5-107 Growth Hormone}

IGF-BP3 is a good predictor of response to GH and rhIGF-1 in non-GHD patients with low IGF-1

\section{level}

Oksana Lazareva; Sheila Perez; Iuliana Predescu; Shahid Malik;

Amrit Bhangoo; Svetlana Ten

State University of New York, Downstate, Pediatric Endocrinology,

Brooklyn, NY, United States

Background: The role of IGFBP-3 in GH response was shown by the new data that it potentiates IGFR1 receptor signaling. In the PREDICT study in GHD patients, polymorphism of IGFBP3 gene was associated with good response to GH therapy. Both low IGF-1 and low IGF-BP3 (-2 SD) were recognized as markers of more severe phenotype of GHIS.

Objective and hypotheses: To study relationship of baseline IGF-BP3 and IGF-1 levels and response to GH and rhIGF-1 therapy in patients with normal GH secretion and low IGF-1 levels.

Methods: 43 children age 9.07 $\pm 2.75 y r, H t-2.72 \pm 0.7$ SD, IGF-1 $-2.76 \pm 0.58$ SD, who passed GHRH stimulation test $(>15 \mathrm{ng} / \mathrm{ml})$ were included. They were treated with GH $(0.46 \pm 0.1 \mathrm{mg} / \mathrm{kg} / \mathrm{wk})$ for 6 months. Patients with poor response to $\mathrm{GH}$, (growth velocity $(\mathrm{GV})<-1 \mathrm{SD}$ ), were switched to IGF-1 therapy $0.24 \mathrm{mg} / \mathrm{kg} / \mathrm{d}$. According to GV all patients were divided in 3 groups 1- responders to GH, 2- responders to IGF-1, 3- non-responders to either GH/ IGF-1. 
Results: IGF-1 was lower in group 3 compared to group 1 with borderline significance, $\mathrm{p}=0.05$. Group 1 had higher IGBP3, $\triangle \mathrm{IGF}-1$, IGF-1 after GH treatment, $\Delta \mathrm{Ht} \mathrm{SD}$ compared to group 2 and 3 . There was no difference between group 1 and group 2 in IGF-1 SD on GH and $\triangle \mathrm{IGF}-1$ after 6 months of treatment, while IGBP3 and $\triangle \mathrm{Ht}$ SD were higher in group 2 than group 3. IGFBP-3 correlated with GV ( $r=0.47, \mathrm{p}<0.01)$, and inversely correlated with GH peak $(\mathrm{r}=-0.45, \mathrm{p}=0.02)$. GV correlated with $\triangle \mathrm{IGF}-1 \mathrm{SD}(\mathrm{r}=0.37, \mathrm{p}=0.02)$. Conclusions: IGF-BP3 is a good predictor of response to GH and IGF-1 therapy in GHI patients with IGF-1<-2 SD. $\Delta$ IGF-1 after GH can differentiate between groups who can benefit from GH or IGF-1 therapy. In case of low IGFBP-3 and low $\triangle \mathrm{IGF}-1$ response on either therapy was poor.

\begin{tabular}{|c|c|c|c|c|c|c|c|}
\hline Group & $\begin{array}{c}\text { Age } \\
\text { (years) } \\
\text { p=ns }\end{array}$ & $\begin{array}{c}\text { Birth } \\
\text { weight } \\
\text { (kg) } p=n s\end{array}$ & $\begin{array}{c}\text { Ht (SD) } \\
\text { Baseline } \\
p=n s\end{array}$ & $\begin{array}{c}\text { IGF-1 } \\
\text { Baseline } \\
\text { (ng/ml) } \\
\text { p=ns }\end{array}$ & $\begin{array}{c}\text { IGF-1 SDS } \\
p=n s\end{array}$ & $\begin{array}{c}\text { GH peak } \\
(\mathrm{ng} / \mathrm{ml}) \\
\mathrm{p}=\mathrm{ns}\end{array}$ & $\begin{array}{l}\Delta \text { IGFBP-3 } \\
\text { after } \\
\text { treatment }\end{array}$ \\
\hline $\begin{array}{l}\text { Group1 } \\
\text { N: } 23 \\
\text { B: } 14\end{array}$ & $\begin{array}{c}9.28 \\
\pm 2.74\end{array}$ & $\begin{array}{c}3.07 \\
\pm 0.35\end{array}$ & $\begin{array}{l}(-2.57) \\
\pm 0.44\end{array}$ & $\begin{array}{l}85.08 \\
\pm 28.3\end{array}$ & $\begin{array}{c}(-2.69) \\
\pm 0.5\end{array}$ & $\begin{array}{l}43.85 \\
\pm 26.2\end{array}$ & $\begin{array}{c}1.02 \\
\pm 1.41\end{array}$ \\
\hline $\begin{array}{l}\text { Group2 } \\
\text { N: } 14 \\
\text { B: } 10\end{array}$ & $\begin{array}{r}9.08 \\
\pm 2.71\end{array}$ & $\begin{array}{c}2.8 \\
\pm 0.48\end{array}$ & $\begin{array}{c}(-2.59) \\
\pm 0.48\end{array}$ & $\begin{array}{l}91.13 \\
\pm 37.2\end{array}$ & $\begin{array}{l}(-2.8) \\
\pm 0.36\end{array}$ & $\begin{array}{c}48.6 \\
\pm 42.7\end{array}$ & $\begin{array}{c}1.26 \\
\pm 1.06\end{array}$ \\
\hline $\begin{array}{l}\text { Group1 } \\
\text { N: } 6 \\
\text { B: } 5\end{array}$ & $\begin{array}{l}8.19 \\
\pm 3.2\end{array}$ & $\begin{array}{c}3.92 \\
\pm 0.43\end{array}$ & $\begin{array}{c}(-3.61) \\
\pm 1.27\end{array}$ & $\begin{array}{c}62 \\
\pm 31.2\end{array}$ & $\begin{array}{l}(-3.48) \\
\pm 0.84\end{array}$ & $\begin{array}{c}77 \\
\pm 82\end{array}$ & $\begin{array}{c}0.63 \\
\pm 0.48\end{array}$ \\
\hline
\end{tabular}

\begin{tabular}{|c|c|c|c|c|c|c|}
\hline Group & $\begin{array}{l}\text { IGFBP-3 } \\
\text { Baseline }\end{array}$ & $\begin{array}{l}\text { IGF-1 } \\
\text { on GH }\end{array}$ & $\begin{array}{l}\text { IGF-1 SD } \\
\text { on GH }\end{array}$ & $\Delta \mathrm{IGF}-1$ & $\begin{array}{c}\Delta \mathrm{IGF}-1 \\
\mathrm{SD}\end{array}$ & $\begin{array}{c}\Delta \mathrm{Ht} \text { SD } \\
\text { after } \\
\text { treatment }\end{array}$ \\
\hline Group 1 & $\begin{array}{c}0.32 \\
\pm 1.18^{\#}\end{array}$ & $\begin{array}{c}301 \\
\pm 114^{\#}\end{array}$ & $\begin{array}{c}1.66 \\
\pm 2.38^{\# \$}\end{array}$ & $\begin{array}{c}216.72 \\
\pm 119.3^{\# \$}\end{array}$ & $\begin{array}{c}4.34 \\
\pm 2.6^{\# \$}\end{array}$ & $\begin{array}{c}0.45 \\
\pm 0.31^{\#}\end{array}$ \\
\hline Group 2 & $\begin{array}{l}-0.46 \\
\pm 1.40^{*}\end{array}$ & $\begin{array}{l}197 \\
\pm 95^{*}\end{array}$ & $\begin{array}{c}-049 \\
\pm 1.68^{\$}\end{array}$ & $\begin{array}{l}101.73 \\
\pm 69.8^{\$}\end{array}$ & $\begin{array}{c}2.08 \\
\pm 1.48^{\$}\end{array}$ & $\begin{array}{c}0.42 \\
\pm 0.35^{\star}\end{array}$ \\
\hline Group 3 & $\begin{array}{c}-2.08 \\
\pm 1.36^{\star \#}\end{array}$ & $\begin{array}{c}97 \\
\pm 44^{\# *}\end{array}$ & $\begin{array}{l}-1.96 \\
\pm 1.32^{\#}\end{array}$ & $\begin{array}{c}35 \\
\pm 26.2^{\#}\end{array}$ & $\begin{array}{c}1.52 \\
\pm 1.22^{\#}\end{array}$ & $\begin{array}{c}0.11 \\
\pm 0.15^{\text {*\# }}\end{array}$ \\
\hline
\end{tabular}

${ }^{*} \mathrm{P}<0.05$ between 1 and 3

$* \mathrm{P}<0.05$ between 2 and 3

${ }^{\$} \mathrm{P}<0.05$ between 1 and 2
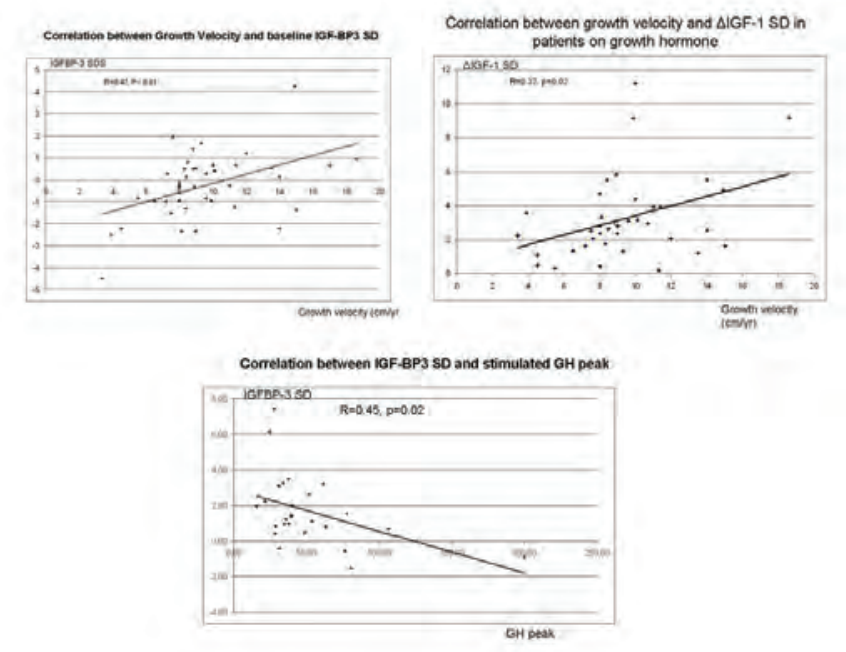

\section{FC5-108 Growth Hormone}

The role of the IGF-1 generation test in assessing children with short stature in current clinical practice: a survey of ESPE members Helena Gleeson'; Regis Coutant'; Mireille Bonnemaire ${ }^{3}$; Helmut Dorr"; Jesús Argente ${ }^{5}$

${ }^{1}$ Leicester Royal Infirmary, Endocrinology, Leicester, United Kingdom; 2Pôle Enfant, $\mathrm{CHU}$ Angers, Endocrinologie Diabétologie Pédiatrique, Angers, France; ${ }^{3}$ psen Pharma SAS, Boulogne Billancourt, France; ${ }^{4}$ Universität Erlangen-Nürnberg, Klinik mit Poliklinik für Kinder und Jugendliche, Erlangen, Germany; ${ }^{5} \mathrm{Hospital}$ Infantil Universitario Niño Jesús, Department of Endocrinology, Madrid, Spain

Background: The IGF-1 Generation Test (IGFGT) has been used to identify children with GH Insensitivity (GHI) Syndrome, the extreme form of severe primary IGF-1 deficiency (IGFD). The literature suggests that the role of the IGFGT in diagnosing partial GHI is unproved. Despite this evidence base, the use of the IGFGT has been increasing since the approval of recombinant human IGF-1 (rhIGF-1) for the treatment of short stature in children with severe primary IGFD.

Objective: To examine the role of the IGFGT in current clinical practice.

Population and method: A survey of 115 paediatric endocrinologists from 30 countries was carried out between 24 August 2010 and 8 September 2010, under the auspices of the European Society for Paediatric Endocrinology (ESPE).

Results: 91 (79\%) respondents declared that they had used the IGFGT in the past two years, mostly to diagnose GHI. The main patient selection criteria were short stature (defined by the endocrinologist as $<-2,<-2.5$, or $<-3$ SDS), low IGF-1 levels and a normal or high GH response to GH provocative tests. A range of different protocols were described. The results of the IGFGT were declared to impact treatment decisions made by 76 of 78 (97\%) respondents and to influence the initiation of rhIGF-1 treatment by 63 of $75(84 \%)$ respondents. The IGFGT was compulsory for obtaining access to rhIGF-1 for 34 of 75 (45\%) respondents.

Conclusions: Despite the evidence base suggesting an unproved role for the IGFGT in diagnosing partial GHI, this survey demonstrates that in current clinical practice the IGFGT is continuing to have an impact on treatment decisions for the majority of paediatric endocrinologists and in some countries dictating access to treatment with rhIGF-1. We suggest that paediatric endocrinologists should develop a consensus as to the optimal pragmatic approach to investigating children with short stature who may have severe primary IGFD and may benefit from a trial of rhIGF-1.

\section{FC5-109 Growth Hormone \\ Contribution of ghrelin receptor (GHSR) mutations to short stature in humans - identification of new variants}

Marie Legendre ${ }^{\prime}$; Jacques Pantef; Cecilia Lazea ${ }^{3}$; Sylvie Cabrof'; Nathalie Collot ${ }^{5}$; Florence Dastot'; Philippe Duquesnoy²; Latifa Hilal Abdelkrim Kadiri; Clémentine Dupuis ${ }^{8}$; Sylvie Nivot ${ }^{9}$; Sophie Rose ${ }^{5}$; Anya Rothenbuhler ${ }^{10}$; Marie-Laure Sobrier ${ }^{2}$; Marie-Pierre Vie-Luton ${ }^{2}$; Yves Le Bouc ${ }^{4}$; Michel Polak ${ }^{11}$; Juliane Léger ${ }^{12}$; Serge Amselem ${ }^{13}$ ${ }^{1}$ APHP and Inserm U933, Hôpital Trousseau, Génétique moléculaire, Paris, France; 'Inserm U933, Génétique, Génomique et Bioinformatique, Paris, France; ${ }^{3}$ Clinica Pediatrie I, Clinica Pediatrie I, Cluj-Napoca, Romania; ${ }^{4}$ APHP, Hôpital Trousseau, Explorations fonctionnelles endocriniennes, Paris, France; ${ }^{5}$ APHP, Hôpital Trousseau, Génétique moléculaire, Paris, France; ${ }^{6}$ Faculté des Sciences, Laboratoire de Génétique et Biologie moléculaire, Kenitra, Morocco; ${ }^{7} \mathrm{CHU}$ Ibn Sina, Service de Pédiatrie, Rabat, Morocco; ${ }^{8} \mathrm{CHU}$ de Grenoble, Service de Pédiatrie, Grenoble, France; ${ }^{9} \mathrm{CHU}$ de Caen, Service de Pédiatrie A, Caen, France; ${ }^{10} \mathrm{APHP}$, Hôpital Saint-Vincentde-Paul, Service d'Endocrinologie pédiatrique, Paris, France; ${ }^{11} \mathrm{APHP}$, Hôpital Necker, Service d'Endocrinologie pédiatrique, Paris, France; ${ }^{12}$ APHP, Hôpital Robert Debré, Service d’Endocrinologie pédiatrique, Paris, France; ${ }^{13} \mathrm{APHP}$ and Inserm U933 (Hôpital Trousseau), Université Pierre et Marie Curie, Génétique moléculaire, Paris, France

Background: Ghrelin, a ligand of the growth hormone (GH) secretagogue receptor (GHSR), is mainly known as the sole orexigenic hormone and as a strong stimulator of GH secretion. Surprisingly, murine models in which the ghrelin axis - i.e. ghrelin or its receptor - is disrupted do not show a pronounced lean and short phenotype, but mainly display subtle changes in glucose and lipid metabolism. So far, very few GHSR mutations have been implicated in short stature with or without altered GH secretion tests.

Objective and hypotheses: The objective if this study is (i) to further test the contribution of GHSR to isolated GH deficit (IGHD) and idiopathic short stature (ISS) and (ii) to provide a most complete description of the phenotype associated with each GHSR variation.

Methods: We screened the GHSR in 290 independent children with ISS or IGHD. We assessed the phenotype (height, weight, somatotrope investigation) of subjects carrying a GHSR variation. Constitutive activity, ghrelin response and cell surface expression of the new variants were assessed through appropriate in vitro assays.

Results: Nine molecular defects were identified: in addition to three previously described mutations (A204E, W2X and R237W), we found six novel variations in the heterozygous state in six probands presenting with ISS $(n=1)$ or IGHD $(n=5)$. According to the functional studies that are underway, those GHSR mutations should account for 3 to $5 \%$ of the short stature phenotype in 
the study group. Except for W2X and R237W, short stature was transmitted in a dominant manner with incomplete penetrance. Variable expressivity of the disease phenotype was noticed. Several of the new variants so far studied showed an impaired constitutive activity associated or not with decreased cell surface expression.

Conclusions: This study, which strengthens the importance of GHSR constitutive activity in human growth, shows that GHSR mutations are responsible for at most $5 \%$ of short stature in humans.

\section{FC5-110 Growth Hormone}

\section{Genetic variations in candidate genes in idiopathic short stature subjects from the epigrow study: analysis of cases versus controls}

Mireille Bonnemaire'; Arunthi Thiagalingam²; Emmanuel Pham ${ }^{1}$, Laurent Naudin'; Zhidong Zhang'; Jeong Rih'; Ron Rosenfeld ${ }^{2}$; Pascale Dutailly ${ }^{4}$; Andrew Grupe ; David Wolfson ${ }^{5}$; Alla Smolgovsky ${ }^{5}$; Joseph Catanese ${ }^{5}$; Charles Rowland ${ }^{5}$; Patrice Denefle ${ }^{1}$; Peter Clayton ${ }^{6}$ ${ }^{1}$ Ipsen Pharma SAS, Translational Sciences, Boulogne-Billancourt, France; ${ }^{2}$ Ipsen Biomeasure Inc, Translational Sciences, Milford, United States; ${ }^{3}$ Oregon Health and Science University, Pediatrics, Portland, United States; ${ }^{4}$ Ipsen Pharma SAS, Global Medical Affairs, BoulogneBillancourt, France; ${ }^{5}$ Celera Corporation, Discovery Research, Alameda, United States; ${ }^{6}$ Royal Manchester Children's Hospital, Paediatric Endocrinology, Manchester, United Kingdom

Objectives and hypothesis: Stature is one of the most heritable human traits, yet many children with a height SDS <-2 are labelled as idiopathic short stature (ISS). A small number of molecular defects in genes associated with the GH-IGF axis have been found in ISS patients. The objective of this study was to identify potentially damaging single nucleotide polymorphisms (SNPs) on 9 genes known to cause growth disorders in ISS versus controls.

Methods: This work was done in the context of the Epigrow epidemiology study, which is a prospective epidemiological study conducted in 9 European countries. Short children were included if they had a normal GH level and no identified cause of SS. Next-Gen deep re-sequencing was performed on exons, exon-intron junctions and promoter regions of the genes in 263 Epigrow subjects and 263 controls. Missense and nonsense SNPs in GH1, GHR, STAT5B, IGF1, IGF1R, IGFALS, CUL7, OBSL1, SHOX were identified as possibly related to SS if they were homozygous in cases but heterozygous or absent in controls, or heterozygotes in cases but absent in controls.

Results: 1356 SNPs were genotyped, 141/0 were found to code for missense/ nonsense variations in cases. 65 SNPs, mainly singletons, fitted the selection criteria: 63 were novel variants, 2 were known GHR mutations. There were no SNPs that met the criteria in the SHOX and IGF1 genes. 55 patients ( 21\%) carried a single mutation and 7 patients ( 3\%) carried 2 or more "case-only" mutations in the 9 genes analyzed.

\begin{tabular}{lcc} 
Gene & Case-only SNPs & Case-only SNPs \\
\hline GH1 & Ho & He \\
GHR & 1 & 6 \\
STAT5B & 0 & 4 \\
IGF1 & 0 & 3 \\
IGF1R & 0 & 0 \\
\hline IGFALS & 0 & 11 \\
CUL7 & 0 & 7 \\
OBSL1 & 2 & 11 \\
SHOX & 4 & 16 \\
\hline & 0 & 0
\end{tabular}

Conclusions: This large well-characterised cohort of ISS patients with a matched number of controls has revealed that 62 patients (24\%) carry potentially damaging mutations in 9 growth attenuating genes. The importance of filtering SNPs not only by possible impact on protein function, but also by comparison to controls is clearly illustrated. It is likely that this cohort will harbour further mutations in other growth-related genes.

\section{FC6-111 Sexual Development \\ Exome sequencing reveals novel genetic causes of 46,XY Disorders of Sex Development (DSD)}

Anu Bashamboo ${ }^{1}$; Vasiliki Karageorgou'; Diana Lourenco'; Joelle Bignon-Topolavic ; Raja Brauner ${ }^{2}$; Ken McElreavey ${ }^{1}$

${ }^{1}$ Institut Pasteur, Human Developmental Genetics, Paris, France; ${ }^{2}$ University Paris Decartes and AP-HP, Hôpital Bicêtre, Pediatric Endocrinology Unit, Le Kremlin Bicêtre, France

Background: Exome sequencing has emerged as a very powerful tool to identify the genetic basis of rare human Mendelian disorders. This approach is particularly attractive for mutation detection in cases of Disorder of Sex Development (DSD) since these conditions are refractory to classic genetic approaches.

Objective and hypotheses: To identify new genetic causes of DSD, we performed exome sequencing in five cases of 46,XY DSD with and without somatic anomalies.

Methods: Following exon enrichment, sequencing was performed using SOliD protocols (Life Sciences). Reads were mapped to the reference Human genome (GRCh37/hg19; 171,744 exons) and subjected to two different computational pipelines: smallIndel and diBayes. This procedure was repeated using data from the 1000 genomes project. Variants were analysed using the EnsEMBL SNP Effect Predictor. Potentially pathogenic variants were confirmed by Sanger sequencing. Functional studies were performed for some variants. Results: Overall, there was an accurate analyses of $96 \%$ of the Agilent High Quality Exome with >x20 coverage. The average coverage was x200. Several pathogenic variants were identified. One 46,XY patient with ambiguous external genitalia and learning difficulties was found to carry two independent mutations in the FOG2 gene. The first is a homozygous p.M544I amino acid change that was inherited from her normal parents, who are first cousins. The second is a de novo heterozygous p.R260E mutation. Both mutations were absent in >300 ancestry-matched controls. In-vitro analysis of the biological activity of the mutant proteins revealed that the protein carrying both the mutations exhibited a quantitative and qualitative reduction in its function, suggesting that both mutations together contribute to the phenotype.

Conclusions: We identified mutations in FOG2 as a novel genetic cause of human 46,XY DSD. This demonstrates the power of exome sequencing to detect pathogenic mutations in patients with DSD.

\section{FC6-112 Sexual Development \\ Long-range regulatory elements of SOX9 in 46,XX testicular DSD}

Jacqueline Hewitt' ${ }^{1}$ Vincent Corbin'; ${ }^{2}$ Thomas Ohnesorg;

Lee Wong'; Vu Chi Dung;; Ngo Diem Ngoc'; Bui Phuong Thao4;

Can Thi Bich Ngoc'; Phuong Mai Nguyen ${ }^{4}$; Nguyen Thanh Liem;

Anthony Papenfuss' ${ }^{2}$; Pascal Philibert ${ }^{5}$ Charles Sultan ${ }^{5}$;

Garry Warne'; Andrew Sinclair ${ }^{3}$

${ }^{1}$ Royal Children's Hospital Melbourne, Endocrinology and Diabetes, Melbourne, Australia; ${ }^{2}$ Walter and Eliza Hall Institute, Bioinformatics,

Melbourne, Australia; ${ }^{3}$ Murdoch Childrens Research Institute,

Molecular Development, Melbourne, Australia; ${ }^{4}$ National Hospital

of Pediatrics, Endocrinology, Hanoi, Vietnam; ${ }^{5}$ Hopital Lapeyronie,

Service d'Hormonologie, Montpellier, France

Background: Understanding the pathogenesis of gonadal dysgenesis in disorders of sex development (DSD) requires knowledge of the sex determination gene cascade and its regulation, however present insights remain incomplete. Testis development requires key upregulation of the conserved SOX9 gene and its downstream gene network, which is thought to occur via an undefined mechanism of SRY in a 46,XY individual. Recent scientific focus has shifted to gonad-specific regulatory elements which surround the SOX9 gene. Objective and hypotheses: We are using whole genome and exome analysis in children with DSD, in order to understand gonad development and disease pathogenesis.

Methods: High-density Illumina 2.5M whole genome SNP microarray was performed on 30 patients; 21 with isolated 46,XY gonadal dysgenesis and 9 with $46, X X$ testicular DSD. Exome sequence capture is performed on patients with 46,XY gonadal dysgenesis.

Results: In patients with 46,XX testicular DSD, duplications in regions $600 \mathrm{~kb}$ upstream of SOX9 were found in 2 of 9 patients (22\%). The duplications span $70 \mathrm{~kb}$ and $340 \mathrm{~kb}$. These small regions align with a currently reported large 
duplication upstream of SOX9 in familial 46,XX testicular DSD, and significantly narrow the candidate locus for a human testis-specific enhancer which could operate in the absence of SRY. The duplications were confirmed by MLPA, and are in tandem arrangement on FISH analysis of both individuals, implicating either a dosage-related or structural effect on SOX9 expression. Bioinformatic analysis of the duplicated regions compellingly supports the presence of a testis-specific enhancer which includes an SRY/SOX binding motif. This candidate enhancer is being functionally assessed by luciferase assay and in mouse and chick models.

Conclusions: These findings address key issues in sex determination: the fundamental regulation of SOX9 and its potential upregulation by SRY, and the causation of 46,XX testicular DSD. On a broader level, they also increase our understanding of the complex system of gene regulation during human development.

\section{FC6-113 Sexual Development \\ The epigenotype of androgen insensitivity syndrome \\ Ole Ammerpohl'; Susanne Bens ${ }^{1}$; Mahesh Appari'; Ralf Werner ${ }^{3}$. \\ Sten Drop ${ }^{4}$; Frans Verheijen ; Yvonne van der Zwan'; leuan Hughes \\ Trevor Bunch ${ }^{6}$; Martine Cools ${ }^{7}$; Olaf Hiort ${ }^{3}$; Reiner Siebert ${ }^{1}$; Paul-Martin Holterhus ${ }^{2}$ \\ ${ }^{1}$ Christian-Albrechts-University of Kiel, Institute of Human Genetics, Kiel, Germany; ${ }^{2}$ Christian-Albrechts-University of Kiel, Department of Paediatrics, Kiel, Germany; ${ }^{3}$ University of Lübeck, Department of Paediatrics, Lübeck, Germany; ${ }^{4}$ Erasmus MC Rotterdam, Sophia Children's Hospital, Rotterdam, Netherlands; ${ }^{5}$ Erasmus MC Rotterdam Department of Clinical Genetics, Rotterdam, Netherlands; ${ }^{6}$ University of Cambridge, Addenbrooke's Hospital, Department of Paediatrics, Cambridge, United Kingdom; ${ }^{7}$ Ghent University Hospital, Department of Endocrinology and Center for Sexology and Genderproblems, Ghent, Belgium}

Background: Sex-specific development of the human is associated with implementation of irreversible sexual dimorphisms of anatomy and function (e.g., differentiation of external genitalia during embryogenesis, pubertal voice change during puberty). Presence or absence of androgens acting via the androgen receptor (AR) plays a key role therein. In single cells, androgens induce short-term changes of gene transcription. In contrast, our genome-wide expression analyses on cultured genital fibroblasts and blood mononuclear cells have shown that androgens have also imprinted long-term changes of the transcriptome ("transcriptional androgen memory").

Objective and hypotheses: To investigate the epigenomic background of AR-dependent sexual dimorphism of external genital differentiation in normal males compared with 46,XY patients having androgen insensitivity syndrome (AIS).

Methods: Genomic DNA was established from labioscrotal fibroblasts of 10 normal males and 28 AIS-patients (CAIS, $\mathrm{N}=17$ ). Methylation profiles were assessed in duplicate by HumanMethylation27 BeadChips containing 27,578 CpG loci (app. 14,000 human genes).

Results: Two different epigenomic patterns were identified. The first pattern showed significant baseline differences of promoter methylation comparing normal males with CAIS (q=0.05) (e.g. PDCD6IP, SSTR3, HSD17B4, PECAM1). The second pattern showed strikingly constant promoter methylation in controls but high variability in the presence of AR-gene mutations (e.g. HOXA5, HOXC8, KRT6A, BMP15). We have confirmed this observation by pyrosequencing the HOXA5 promoter. Changes in pattern 2 were unpredictable by the degree of genital virilization alone and vice versa.

Conclusions: Our data indicate that androgens have set specific methylation marks in sexually dimorphic genital tissue confirming "androgen memory" at the epigenome level. Moreover, disruption of the AR-pathway also induces variability of methylation patterns. We propose that this "AIS-epigenotype" could be a functional link to overt genotype-phenotype diversity in AIS.

\section{FC6-114 Sexual Development \\ A loss-of-function missense mutation in the TALE Homeodomain protein PBX1 associated with 46,XY DSD}

Anu Bashamboo'; Raja Brauner ${ }^{2}$; Diana Lourenco ${ }^{1}$; Oliver Zwermann ${ }^{3}$; Urs Lichtenauer'; Felix Beuschlein ${ }^{3}$; Ken McElreavey

'Institut Pasteur, Human Developmental Genetics, Paris, France; ${ }^{2}$ University Paris Descartes and AP-HP Hôpital Bicêtre, Pediatric Endocrinology, Le Kremlin Bicêtre, France; ${ }^{3}$ Ludwig-Maximilians University, Endocrine Research Unit, Munich, Germany

Background: Interactions between homeodomain-containing proteins enhance both DNA-binding affinity and specificity in many developmental regulatory networks. HOX gene products act in concert with the PBC class of cofactors that include the PBX1 (Pre-B-cell leukemia homeobox 1) protein. PBX1 is a member of the three-amino acid loop extension class of homeodomain transcription factors. Pbx1-/- mice die in utero with multiple organ hypoplasia or aplasia. Pbx1-/- mice also have impaired testes development. Objective and hypotheses: We tested the hypothesis that mutations involving PBX1 could be associated with 46,XY DSD.

Methods: PBX1 coding sequences were sequenced in a 46,XY girl of French ancestry with complete gonadal dysgenesis. Functional studies, including DNA-binding assays and protein interaction assays were performed using the wild-type and mutant PBX1 proteins.

Results: We identified a de novo p.R235Q heterozygous missense mutation in the highly-conserved PBX1 TALE homeodomain. This mutation did not affect the DNA-binding activities of the protein. Although the mutant protein failed to interact with known protein partners including HOXB8, it retained its ability to interact with another regulatory cofactor, PREP1 that is essential for adrenal development. No sequence variants were observed in PBX1 coding sequences in $>500$ ancestry-matched control samples.

Conclusions: We have identified the first mutation in PBX1 in the human. This mutation had a severe impact on testis development but other major organ systems appeared normal. Differential interaction with various developmental protein partners may explain the lack of major somatic anomalies. This case, together with previous reports of mutations in the genes NR5A1, GATA4, MAP3K1 and CBX2, demonstrate that mutations in pleiotropic genes can specifically impact testis-determination resulting in 46,XY DSD. Such mutations can offer unique insights into the mechanism of gonad formation

\section{FC6-115 Sexual Development \\ The first human cytochrome b5 missense mutation causes 46,XY DSD due to apparent CYP17A1 17,20 lyase deficiency Jan Idkowiak'; Tabitha Randell'; Vivek Dhir'; Pushpa Patel'; Cedric H.L. Shackleton ${ }^{1}$; Nils Krone'; Wiebke Arlt' 'University of Birmingham, CEDAM - Centre for Endocrinology, Diabetes and Metabolism, Birmingham, United Kingdom; ${ }^{2}$ Nottingham University Hospitals NHS Trust, Department of Paediatric Endocrinology, Nottingham, United Kingdom}

Background: In humans, androgen synthesis depends on the enzyme CYP17A1. The 17,20 lyase activity of CYP17A1 catalyses the conversion of 17-hydroxypregnenolone to the universal androgen precursor dehydroepiandrosterone (DHEA). CYP17A1 requires electron transfer from P450 oxidoreductase (POR) for activity. Cytochrome B5 (CYB5) is thought to enhance allosteric interaction of CYP17A1 and POR proteins thereby facilitating CYP17A1 17,20 lyase activity.

Objective and hypotheses: Mutations in CYB5 may explain isolated 17,20 lyase deficiency observed in children from a large consanguineous family. Methods: Steroid hormone analysis by gas chromatography/mass spectrometry, genetic analysis by direct sequencing; functional in vitro characterisation after co-expression of CYP17A1 with wildtype and mutant CYB5, respectively, in HEK293 cells, followed by enzymatic activity assays.

Results: The index patient was a 5-year-old boy who presented with ambiguous genitalia at birth looking predominantly female but with gonads papable in the labia majora. Further family work-up revealed that his 13-year-old sister was lacking pubertal development and a recently born younger brother also had presented with severe undermasculinisation. The karyotype was $46, \mathrm{XY}$ in all three siblings. Serum sex steroids were low; urinary steroid 
profiling revealed low or undetectable androgen metabolites with elevated excretion of 17-hydroxypregnenolone but normal glucocorticoid and mineralocorticoid excretion, indicative of isolated 17,20 lyase deficiency. No mutations were found in CYP17A1 or POR. However, in all three siblings we detected a homozygous CYB5 missense mutation (g.2510A >T) replacing a highly conserved histidine with leucine at amino acid position 44 (p.H44L). The parents were heterozygous for p.H44L. Kinetics in our overexpression system revealed that p.H44L greatly reduces 17,20 lyase activity without affecting CYP17A1 17-hydroxylase activity.

Conclusions: We have identified the first human CYB5 missense mutation as a cause of 46,XY DSD.

\section{FC6-116 Sexual Development}

\section{Aromatase excess syndrome: identification of} cryptic duplications and deletions leading to gain-of-function of CYP19A1 and assessment of phenotypic determinants

Maki Fukami'; Makio Shozu²; Shun Soneda'; Fumiko Kato'; Akemi Inagakiं; Hiroshi Takagi'; Keiichi Hanaki'; Susumu Kanzaki'; Kenji Ohyama ${ }^{6}$; Tomoaki Sano ${ }^{6}$; Toshinori Nishigaki ${ }^{7}$; Susumu Yokoya ${ }^{8}$; Gerhard Binder ${ }^{\theta}$; Reiko Horikawa ${ }^{8}$; Tsutomu Ogata ${ }^{1}$

${ }^{1}$ National Research Institute for Child Health and Development, Department of Molecular Endocrinology, Tokyo, Japan; ${ }^{2} \mathrm{Chiba}$ University, Department of Reproductive Medicine, Chiba, Japan; ${ }^{3}$ Nagoya Second Red Cross Hospital, Department of Diabetes and Endocrinology, Nagoya, Japan; ${ }^{4}$ Tottori University, Department of Women's \& Children's Family Nursing, Yonago, Japan; ${ }^{5}$ Tottori University, Division of Pediatrics and Perinatology, Yonago, Japan; ${ }^{6}$ University of Yamanashi, Department of Pediatrics, Chuo, Japan; ${ }^{7}$ Osaka Police Hospital, Department of Pediatrics, Osaka, Japan; ${ }^{8}$ National Medical Center for Children and Mothers, Department of Medical Subspecialties, Tokyo, Japan; 'University Children's Hospital, Pediatric Endocrinology Section, Tuebingen, Germany

Background: Aromatase excess syndrome (AEXS) is an autosomal dominant disorder characterized by gynecomastia. Although cryptic inversions leading to abnormal fusions between CYP19A1 encoding aromatase and its neighboring genes have been identified in a few patients, the molecular basis remains largely unknown.

Patients: We studied 18 affected males from families A-F. Blood analyses showed elevated estradiol/testosterone ratios and FSH-dominant hypogonadotropic hypogonadism in all cases examined.

Molecular analysis: Comparative genomic hybridization analysis identified a heterozygous genomic rearrangements in all cases; 79,156 bp tandem duplication involving seven of 11 non-coding CYP19A1 exons 1 in families A and $\mathrm{B}$, a 211,631 bp deletion involving exons 2-43 of DMXL2 and exons 5-10 of GLDN in family C, and a 165,901 bp deletion involving exons 2-43 of $D M X L 2$ in families D-F. Transcript analysis revealed that the duplications increased the number of functioning CYP19A1 transcription start sites, and the deletions produced the same chimeric mRNA consisting of DMXL2 exon 1 and CYP19A1 coding exons. The DMXL2 exon 1 harbored a translation start codon, whereas inversion-mediated chimeric mRNAs had no coding sequence on the fused exon 1. CYP19A1 was expressed in a limited number of tissues, whereas its neighboring genes involved in the chimeric mRNA formation were expressed widely. Clinical features were milder in patients with duplications/deletions than in those with inversions.

Conclusions: The present study shows that AEXS can be caused by duplications of the physiological promoters and deletions of the upstream regions of CYP19A1, and that phenotypic severity is primarily determined by the tissue expression pattern of CYP19A1 and the chimeric genes and by structural properties of the fused exons. Thus, this study provides novel models for the gain-of-function mutations leading to human genetic disease.
FC7-117 Cell Growth and Endocrine Oncology

\section{Uneven pattern of methylation at 11 p15 IGF2/H19 locus: a stepwise toward the mechanism of regulation with major diagnostic consequences}

Virginie Steunou'; Salah Azzi; Sylvie Rossignol; Yves Le Bouc; Irene Netchine ${ }^{2}$

${ }^{1}$ INSERM, INSERM U938 team 4, Paris, France; ${ }^{2}$ Pierre and Marie Curie University (UPMC)/Armand Trousseau Hospital, INSERM U938

team 4, Paris, France

Background: Human IGF2/H19 locus at chromosome 11p15.5 harbours several differentially methylated regions (DMR) all methylated on the paternal allele. The Imprinted Center Region 1 (ICR1), which regulates the locus, contains 7 binding sites for the zinc-finger protein CTCF (CBS); 3 additional DMRs are located respectively at H19 promoter (H19DMR) and 2 into IGF2 gene (DMR0 and DMR2). The structural organisation of this locus is very complex and the mechanism underlying its regulation is poorly understood. Loss of imprinting (LOI) at ICR1 H19/IGF2 domain results in two growth disorders with opposite phenotypes: an overgrowth disorder, Beckwith-Wiedemann syndrome [BWS, ICR1 gain of methylation (GOM) in $10 \%$ of cases] and a growth retardation disorder, Silver-Russell syndrome [SRS, ICR1 loss of methylation (LOM) in $60 \%$ of cases].

Objective and hypotheses: Document whether the imprint is similarly apposed among the different CBSs and DMRs in a control population and determine the methylation pattern induced by the LOM of this region in SRS patients.

Methods: DNA from 50 control subject, 73 SRS patients with no molecular aetiology identified and 106 SRS patients with CBS2 ICR1 11p15 LOM were studied using allele specific methylation multiplex real time quantitative PCR (ASMM RTQ-PCR).

Results: The methylation indexes (MI) were balanced at all regions in the control population and SRS patients with no ICR1 $11 \mathrm{p} 15$ defect identified except at CBS4, CBS5 and IGF2 DMR2 where the methylation was variable. Interestingly, some SRS patients with CBS2 LOM showed uneven pattern of methylation among the CBSs, especially, normal MIs at CBS1 and CBS7 were identified.

Conclusions: Our results demonstrate that the methylation establishment at IGF2/H19 locus can be uneven among the CBSs and DMRs. This suggests that CBSs play different roles for the regulation of this locus. These results are very important to take into account to establish an accurate molecular diagnosis of SRS and BWS.

\section{FC7-118 Cell Growth and Endocrine Oncology CDKN1C: new mutations and clinical spectrum} in Beckwith-Wiedemann syndrome

Frederic Brioude ${ }^{1}$; Irene Netchine ${ }^{1}$; Fabienne Danton';

Marilyne Le Jule'; Nathalie Thibaud'; Christine Gicquer

Yves Le Bouc ${ }^{1}$; Sylvie Rossignol'

${ }^{1}$ APHP, Explorations Fonctionnelles Endocriniennes, Laboratoire de Biologie Moléculaire - Hôpital Trousseau, Paris, France; Baker IDI Heart and Diabetes Institute, Epigenetics in Human Health and Disease, Melbourne, Australia

Background: Beckwith-Wiedemann syndrome (BWS) is an overgrowth syndrome associated with macroglossia, abdominal wall defects, visceromegaly and an increased risk of childhood tumor. Molecular anomalies are mostly epigenetic (gain (GOM) or loss (LOM) of methylation at 11p15 locus); however, mutations of CDKN1C have been implicated in sporadic or familial forms of BWS.

Objective and hypotheses: To describe the spectrum of the CDKN1C mutations and the phenotype of the patients in a large cohort of BWS patients.

Methods: The 3 exons of CDKN1C and the intron-exon boundaries were sequenced in patients with normal methylation status at $11 \mathrm{p} 15$ exhibiting abdominal wall defect. Related patients were analyzed only for the fragment implicated in the propositus.

Results: Among the 595 BWS with 11p15 molecular abnormalities, we identified 34 mutations in 36 pedigrees ( 46 patients and 7 fetuses) which represent $6 \%$ of our whole cohort. All types of mutation were detected: four missense, eight nonsense, two deletions or insertions, eighteen frameshift, and two alternative splicing mutations. When parental samples were available, all the 
mutations but two (one de novo and one suspected germinal mutation). were inherited from the mother. It is noteworthy that phenotype of the patients can be variable, even within a same family. Though, the four missense mutations seem to lead to a less severe phenotype regarding the abdominal wall defect than the other mutations. As expected, asymmetry is an extremely uncommon symptom in our patients. Four tumors occurred (one neuroblastoma, one ganglioneuroma, one melanoma and one acute lymphoid leukemia (ALL). CDKN1C mutations lead to a lower frequency of neonatal macrosomia, visceromegaly and tumors when compared with GOM at ICR1 locus.

Conclusions: We present the largest cohort of patients with CDKN1C mutations. Phenotype seems to be extremely variable, making a prenatal diagnosis difficult. Furthermore, we observed two non-embryological tumors (ALL and melanoma) which are uncommon in BWS.

\section{FC7-119 Cell Growth and Endocrine Oncology The SOCS2 KO mouse - a valid model for studying the local effects of GH on bone Ross Dobie'; Carmen Huesa'; Robert van't Hof'; Vicky MacRae'; S. Faisal Ahmed ${ }^{3}$; Colin Farquharson ${ }^{1}$ ${ }^{1}$ Roslin Institute, Developmental Biology, Edinburgh, United Kingdom; ${ }^{2}$ Molecular Medicine Centre, Rheumatic Diseases Unit, Edinburgh, United Kingdom; ${ }^{3}$ Royal Hospital for Sick Children, Bone and Endocrine Research Group, Glasgow, United Kingdom}

Background: Suppressor of Cytokine Signalling 2 (SOCS2) is a major regulator of GH action in chondrocytes and despite normal circulating IGF-1, the knockout mice are characterised by enhanced body size.

Objective and hypotheses: To understand the role of SOCS2 in bone development.

Methods: Micro-CT was carried out on the tibia of wild-type (WT) and SOCS2 knockout (KO) mice, at 6-weeks (juvenile) and 4-months (adult) of age to study trabecular and cortical microarchitecture; the mineral density of the bone tissue was calculated; 3-point-bending was performed and markers of bone turnover were assessed.

Results: A significant increase in body weight was observed in all $\mathrm{KO}$ mice $(\mathrm{P}<0.05)$. KO mice had increased trabecular BV/TV compared to WT controls, a likely consequence of increased trabecular thickness $(\mathrm{P}<0.05)$. Whilst all KO mice had increased tibial periosteal surface area $(\mathrm{P}<0.05)$, the cortex was thicker in male mice at both ages, but not females. The thicker cortex of the male KO mice tibia was associated with increased work to failure. There was no difference in the mineral density of the bone tissue in either juvenile gender however adult male and female $\mathrm{KO}$ mice had a significant decrease of mineral density in the cortical bone $(\mathrm{P}<0.05)$. Juvenile female $\mathrm{KO}$ mice had higher serum osteocalcin compared to WT. Serum carboxy-terminal collagen crosslinks, a marker of bone resorption, was raised in male \& female juvenile $\mathrm{KO}$ mice but this increase only reached significance in the males.

Conclusions: The increased bone size and mass are consistent with the known anabolic effects of local, skeletal, IGF-1 and these effects are age and sex specific. The fall in mineral density of cortical bone reflects the importance of circulating IGF-1 on cortical bone development. The SOCS2 KO mouse represents a valid model for studying the local effects of GH and IGF-1 on bone.

\section{FC7-120 Cell Growth and Endocrine Oncology \\ Influences of down-regulation expression of SOCS3 by siRNA on post insulin-receptor signal pathway of catch-up IUGR rats in vitro \\ Juan Ye; Xiaoping Luo; RD Zheng; YQ Ying}

Tongji Hospital, Tongji Medical College, Huazhong University of Science and Technology, Department of Pediatrics, Wuhan, China

Background: Epidemiological data have shown that IUGR,low birth weight and later obesity are highly correlated with development of insulin resistance and type 2 diabetes. Most of IUGR individuals have catch-up growth after birth, but those with weight catch-up growth are more prone to insulin resistance. SOCS3 is an important negative regulatory factor involved in pathogenesis of insulin resistance, high expression of SOCS3 levels aggravates insulin resistance.

Objective: To elucidate influences of down regulation of SOCS3 by siRNA on post insulin-receptor signal pathway of catch-up IUGR rats in vitro.

Methods: IUGR rat model was established by maternal nutrition restriction.
Rat hepatocytes were isolated and cultured using in situ two-step perfusion method.SiRNA aimed at SOCS3 was transfected to hepatocytes by liposome.mRNA levels of molecules involved in post insulin-receptor signaling pathway were measured among catch-up IUGR group(IG), post-transfection catch-up IUGR group(PG) and AGA group(AG) by realtime PCR. Western blotting was used to detect protein and protein phosphorylation changes.

Results: We successfully established catch-up IUGR rat model and cultured primary rat hepatocytes.Before insulin stimulation mRNA expression levels of molecules involved in post insulin-receptor signaling pathway [IRS-1,IRS2,PI3K $(\mathrm{p}<0.05)$; PKB $(\mathrm{p}>0.05)]$ were in IG lower than in AG, after insulin stimulation reduction is more obvious $(\mathrm{p}<0.05)$; compared with IG, the mRNA levels increased in PG before insulin stimulated [IRS-1,IRS-2,PI3K $(\mathrm{p}<0.05)$; PKB $(\mathrm{p}>0.05)$ ] and after insulin stimulated [IRS-1, PKB, PI3K $(\mathrm{p}<0.05)$; IRS-2 ( $>0.05)]$; compared with AG, the mRNA levels decreased in PG before insulin stimulated [IRS-1, IRS-2, PKB ( $p>0.05)$; PI3K $(\mathrm{p}<0.05)$ ] and after insulin stimulated [PI3K, IRS-2, PKB $(\mathrm{p}>0.05)$; IRS-1 $(\mathrm{p}<0.05)]$; Protein levels were consistent with the mRNA levels. P-Akt2 level was higher in PG than in IG, but with no difference in AG.

Conclusions: Down regulation of SOCS3 can improve insulin resistance of catch-up IUGR rats in vitro through enhancing post insulin-receptor pathway signal transduction.

\section{FC7-121 Cell Growth and Endocrine Oncology Abnormal activation of the Wnt/ $\mathbf{\beta}$-catenin pathway in childhood adrenocortical tumors (ACTs) \\ Leticia Ferro Leal' ; Livia Mara Mermejo²; Leandra Z Ramalho3; Carlos Eduardo Martinelli $\mathrm{Jr}^{1}$; Jose Andres Yunes"; \\ Ana Luiza Seidinger $r^{4}$ Maria Jose Mastellaro4; Silvia Regina Brandalise"; Ayrton Moreira²; Luiz Gonzaga Tone'; Carlos Alberto Scrideli'; Margaret Castro'; Sonir R. Antonini' ${ }^{1}$ School of Medicine of Ribeirao Preto, University of Sao Paulo, Pediatrics, Ribeirao Preto, Brazil; ${ }^{2}$ School of Medicine of Ribeirao Preto, University of Sao Paulo, Internal Medicine, Ribeirao Preto, Brazil; ${ }^{3}$ School of Medicine of Ribeirao Preto, University of Sao Paulo, Pathology, Ribeirao Preto, Brazil; ${ }^{4}$ Boldrini Children's Center, Pediatric Oncology, Campinas, Brazil}

Background: Activation of $\mathrm{Wnt} / \beta$-catenin pathway is frequent in adult ACTs). Data on childhood ACTs are lacking.

Objective and hypotheses: To investigate abnormalities of the Wnt/ $\beta$ catenin pathway and CTNNB1 mutations in childhood ACTs.

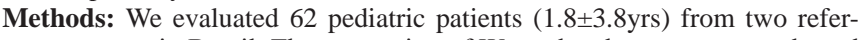
ences centers in Brazil. The expression of Wnt-related genes were evaluated by qPCR and IHC.

Results: Overall survival (OS) was associated with tumoral stage $(\mathrm{p}<0.0001)$ and age at diagnosis $<5 \mathrm{yrs}(\mathrm{p}<0.0001)$. The p.R337H P53 mutation was found in $89 \%$ ACTs and was not associated with clinicopathological findings or outcome. CTNNB1 mutations were found in 6\% ACTs, also harboring the TP53 mutation, and it was associated with lower OS $(\mathrm{p}=0.03)$ and higher death rate $(\mathrm{p}=0.02)$. We found decreased mRNA expression of DKK3 (-14-fold; $\mathrm{p}<0.0001)$, WNT4 (-6.1-fold; $\mathrm{p}=0.0083)$, SFRP1 (-4-fold; $\mathrm{p}=0.05)$, TCF1 $(-3-$ fold; $\mathrm{p}=0.007)$, c-MYC $(-2.8$-fold; $\mathrm{p}=0.03)$ and Axin $(-1.6$-fold; $\mathrm{p}=0.04)$ and overexpression of CTNNB1 mRNA (1.9-fold; $\mathrm{p}=0.008$ ). WNT4 and TCF1 underexpression were more pronounced in ACTs stages I-II (-13.9-fold, $\mathrm{p}=0.02$; -4.9 -fold, $\mathrm{p}=0.006$, respectively). TP53 mRNA levels were lower in ACTs harboring TP53 mutation than in nonmutated tumors (-2.3-fold; $\mathrm{p}=0.04$ ). IHC showed increased cytoplasmic and/or nuclear $\beta$-catenin accumulation in $68 \%$ of the ACTs, none harboring CTNNB1 mutations. Moderate or strong P53 cytoplasmic and/or nuclear accumulation was observed in $40 \%$ of the tumors. There was a positive correlation between $\beta$-catenin and P53 accumulation ( $\mathrm{r}=0.55 ; \mathrm{p}=0.009)$. DKK3 and c-MYC immunostaining were low or absent in $58 \%$ and $67 \%$ of the ACTs, respectively.

Conclusions: CTNNB1 mutations are rare and are associated with poor prognosis in childhood ACTs harboring the TP53 mutation. Wnt/ $\beta$-catenin pathway is deregulated in childhood ACTs but not due to CTNNB1 mutations, indicating the existence of additional mechanisms underlying activation of this pathway. The downregulation of Wnt inhibitor genes, such as DKK3, SFRP1 and Axin, may trigger this activation. 
FC7-122 Cell Growth and Endocrine Oncology

Longitudinal assessment of cardiovascular risk factors (CVRF) in adult survivors of pediatric cancer: a report from the childhood cancer survivor study (CCSS)

Lillian Meacham ${ }^{1}$; Greg Armstrong'; Yan Chen³; Toana Kawashima Charles Sklar ; Daniel Mulrooney ${ }^{6}$; Eric Chow ${ }^{7}$; William Border'; Jean Durand ${ }^{\beta}$; Ann Mertens ${ }^{1}$; Marilyn Stovall ${ }^{\beta}$; Wendy Leisenring ${ }^{4}$; Yutaka Yasui ${ }^{3}$; Leslie Robison ${ }^{2}$; Kevin Oeffinger ${ }^{10}$

'Emory University/Children's Healthcare of Atlanta, Pediatrics, Atlanta, United States; ${ }^{2}$ St Jude Children's Research Hospital, Epidemiology and Cancer Control, Memphis, United States; ${ }^{3}$ University of Alberta, Public Health Sciences, Edmonton, Canada; ${ }^{4}$ Fred Hutchinson Cancer Rsrch Ctr, Clinical Research and Public Health Science Divisions, Seattle, United States; ${ }^{5}$ Memorial Sloan-Kettering Cancer Center, Pediatrics, New York, United States; ${ }^{6}$ University of Minnesota Medical School and Cancer Ctr, Pediatrics, Minneapolis, United States; ${ }^{7}$ Fred Hutchinson Cancer Rsrch Ctr, Pediatrics, Seattle, United States; ${ }^{8} \mathrm{MD}$ Anderson Cancer Ctr, Cardiology, Houston, United States; ${ }^{9} \mathrm{MD}$ Anderson Cancer Ctr, Radiation Physics, Houston, United States; ${ }^{10}$ Memorial Sloan-Kettering Cancer Center, Medicine and Pediatrics, New York, United States

Background: Childhood cancer survivors are at increased risk of having individual and multiple cardiovascular risk factors (CVRF).

Objective and hypotheses: We estimated the prevalence of CVRFs with increasing age in survivors as compared to sibling controls.

Methods: Analyses included 13,268 $\geq 5$-yr survivors of childhood cancer diagnosed between 1970-86 and followed longitudinally over 15 yrs (median age at last follow-up 31.9 yrs, range 5.6-58.9). Prevalence of obesity (body mass index BMI $\geq 30 \mathrm{~kg} / \mathrm{m}^{2}$ ) and CVRFs requiring medical treatment (diabetes, hypertension and dyslipidemia) were ascertained at baseline and through three follow-up surveys for survivors and a comparison sibling cohort $(n=4,001)$. Associations between CVRFs and demographic and treatment variables were assessed.

Results: Prevalence of individual and multiple ( $\geq 3$ ) CVRFs increased with age in both survivors and siblings. Prevalence odds ratios (ORs) and $95 \%$ confidence intervals (CIs) for CVRFs in survivors compared to siblings:

\begin{tabular}{|lccccc} 
& $\begin{array}{c}\text { BMI } \mathbf{3 0} \\
\mathbf{k g} / \mathbf{m} 2\end{array}$ & \multicolumn{2}{c}{ Hypertension Dyslipidemia } & $\begin{array}{c}\text { Diabetes } \\
\text { Mellitus }\end{array}$ & $\geq \mathbf{3}$ CVRF \\
\hline Age & OR $(95 \% \mathrm{Cl})$ & OR $(95 \% \mathrm{Cl})$ & $\mathrm{OR}(95 \% \mathrm{Cl})$ & $\mathrm{OR}(95 \% \mathrm{Cl})$ & $\mathrm{OR}(95 \% \mathrm{Cl})$ \\
\hline $\begin{array}{l}<30 \\
\text { years }\end{array}$ & $1.1(1.0-1.2)$ & $2.7(2.3-3.2)$ & $3.3(2.4-4.6)$ & $1.9(1.4-2.7)$ & $1.9(1.1-3.6)$ \\
\hline $\begin{array}{l}30-39 \\
\text { years }\end{array}$ & $1.0(0.9-1.1)$ & $2.5(2.1-2.8)$ & $2.7(2.3-3.3)$ & $2.1(1.6-2.6)$ & $2.3(1.7-3.2)$ \\
$\begin{array}{l}40-49 \\
\text { years }\end{array}$ & $0.9(0.9-1.0)$ & $2.3(2.0-2.6)$ & $2.4(2.1-2.9)$ & $1.9(1.5-2.4)$ & $2.1(1.6-2.7)$ \\
\hline $\begin{array}{l}250 \\
\text { years }\end{array}$ & $0.8(0.6-1.0)$ & $2.3(1.8-2.9)$ & $1.9(1.5-2.4)$ & $2.1(1.5-2.9)$ & $1.6(1.1-2.3)$ \\
\hline
\end{tabular}

Multiple CVRF ( $\geq 3$ ) were more common in African Americans (OR 2.1; 95\% CI 1.1-3.8) and former smokers (OR 1.6; 95\% CI 1.2-2.2). Total body irradiation (OR 3.0; 95\% CI 1.2-7.7), cranial spinal radiation (OR 2.2; 95\% CI 1.3-3.7) and exposure to alkylating agents (OR 1.5 ; 95\% CI 1.1-2.1) were associated with multiple CVRFs.

Conclusions: In their $3^{\text {rd }}$ and $4^{\text {th }}$ decades, survivors of childhood cancer exhibit individual and multiple CVRFs at higher rates than their siblings; however, the relative odds remained stable or decreased with age.

\section{FC8-123 Diabetes and the Beta Cell \\ Partial transition towards a mesenchymal phenotype allows human pancreatic duct cells to proliferate while showing a beta-cell differentiation potential in vitro}

Philippe Lysy'; Ji Lei;; James Markmann²; Susan Bonner-Weir ${ }^{1}$ 'Joslin Diabetes Center, Harvard Medical School, Islet Transplantation and Cell Biology, Boston, United States; 'Massachusetts General Hospital, Harvard Medical School, Department of Surgery, Boston, United States

Background: Embryonic and induced pluripotent stem cells currently achieve state - of - the - art $\beta$ - cells differentiation levels. However, their ethical and safety limitations stress the need for other cell types with efficient $\beta$ - cell differentiation capacities and unrestricted use.

Objective and hypotheses: To evaluate the in vitro proliferation and differentiation potential of cultures derived from human pancreatic duct cells. Methods: Human CA19.9+ duct cells were isolated from 4 islet- depleted pancreatic tissues using MACS columns. Gene expression was analyzed by quantitative RT-PCR and protein expression was determined by immunostaining and FACS analysis.

Results: Duct cells had limited proliferation in serum—supplemented CMRL medium but when cultured in endothelial growth medium EGM-2-MV, they showed sustained proliferation up to 9 passages. In this medium, some duct cells lost their epithelial morphology, acquired a spindle-shaped appearance and proliferated. With passaging, the human duct-derived cells (HDDCs) lost gene expression of duct markers E-cadherin, CFTR and CAII and increasingly expressed mesenchymal markers vimentin and $\alpha \mathrm{SMA}$; duct markers CK19, Sox9 and HNF1 $\beta$ remained expressed at low levels. HDDCs co-expressed both duct marker CK19 and mesenchymal marker CD90 at first passage, indicating a partial epithelial- to - mesenchymal transition (EMT). Inhibition of EMT with A-83-01 immediately after plating prevented the appearance of HDDCs. Furthermore, HDDCs differed in gene expression and proliferation profiles from human skin fibroblasts and the stromal cells appearing in unselected exocrine cultures, suggesting that HDDCs were unlikely to result from contamination. After a differentiation protocol, HDDCs started to express insulin, $P d x-1$, MafA, GLUT -2 and synaptophy$\sin$ mRNAs. Moreover, $2.1 \pm 0.7 \%$ of the cells differentiated into insulin-expressing cells.

Conclusions: We show that in vitro human CA19.9+ duct cells can evolve towards a mesenchymal phenotype. The resulting highly proliferative cells have potential to $\beta$-cell differentiation, so they bear promise as a tool for diabetes cell therapy.

\section{FC8-124 Diabetes and the Beta Cell \\ Prevalence and clinical characteristics of MODY-type diabetes with onset in childhood and adolescence: analysis of 462 patients from the prospective German-Austrian DPV-survey Klemens Raile ${ }^{1}$; Wendy Awa2; Angelika Thon ${ }^{3}$; Jürgen Grulich-Henn ${ }^{4}$; Thomas Meissner ${ }^{6}$; Edith Schober ${ }^{6}$ : Reinhard Holl \\ ${ }^{1}$ Charité, Paediatric Diabetology, Clinic of Paediatrics, Berlin, Germany; ${ }^{2}$ Faculty of Medicine, University of UIm, Department of Epidemiology, Ulm, Germany; ${ }^{3}$ Hannover Medical School, Paediatrics, Hannover, Germany; ${ }^{4}$ University of Heidelberg, Children's Hospital, Heidelberg, Germany; ${ }^{5}$ University Children's Hospital, Department of General Paediatrics, Düsseldorf, Germany; ${ }^{6}$ Medical University of Vienna, Department of Paediatrics, Vienna, Austria}

Background: To date, mutations of at least 13 genes were known to cause a phenotype observed in MODY-type diabetes. The most frequent types MODY3 and MODY2 have been well characterized in terms of phenotype and outcome but limited data exist on prevalence and clinic first, in other forms of MODY-diabetes and second, in children and adolescents.

Objective and hypotheses: We report on prevalence, genetic diagnosis and clinical characteristics of patients with MODY-type diabetes and diabetes onset earlier than 18 yrs in Germany and Austria.

Patients and methods: This analysis includes 58,914 children, adolescents and young adults from 150 diabetes centres in Germany and Austria with diabetes onset before the age of 18 yrs. Among those, 462 patients ( $0.79 \%)$ were 
entered as having a specific MODY-diagnosis (genetic defect of HNF4A, GCK, HNF1A, PDX1 and HNF1B). All clinical data and genetic testing were prospectively documented in the DPV database by the treating diabetologist. Data analysed here were age, diabetes onset, associated cardiovascular risk factors, diabetes complications, treatment modalities and metabolic control dependent on the MODY-type.

Results: Among the 462 patients with a monogenic diabetes diagnosis $61 \%$ had genetic defects of GCK (MODY2), 31\% of HNF1A (MODY3), 5\% of HNF4A (MODY1), 2\% of HNF1B (MODY5) and 1\% of PDX1 (MODY4). Allthough retinopathy and nephropathy was rare, MODY5 and MODY3 had higher HBA1c-levels and an increased risk profile to develop micro- and macrovascular complications. Along with progression of beta cell failure, insulin treatment was established in all cases with MODY5, approx. 50\% of cases with MODY3 and MODY1, but only 10\% of MODY2 cases.

\begin{tabular}{lccccc} 
MODY-Type & MODY1 & MODY2 & MODY3 & MODY4 & MODY5 \\
\hline Genetic defect & HNF4A & GCK & HNF1A & PDX1 & HNF1B \\
\hline N (All = 462) & $23(5 \%)$ & $282(61 \%)$ & $144(31 \%)$ & $4(1 \%)$ & $9(2 \%)$ \\
\hline male / female & $5 / 18$ & $151 / 131$ & $54 / 90$ & $1 / 3$ & $3 / 6$ \\
\hline Age at diagnosis (yrs) & 12.3 & 9.7 & 11.7 & 11.8 & 13.9 \\
\hline HbA1c (\%) & 6.8 & 6.4 & 7.2 & 6.6 & 8.2 \\
\hline Dyslipidemia (\%) & 23.0 & 31.7 & 44.3 & 66.7 & 80.0 \\
Hypertension (\%) & 14.0 & 6.3 & 11.9 & 0 & 33.3 \\
Overweight (>P90; \%) & 48.0 & 15.0 & 32.0 & 50.0 & 33.3 \\
MA / Ret. (\%) & $0 / 0$ & $0 / 0$ & $5 / 0.8$ & $0 / 0$ & $50 / 0$ \\
\hline Treatment (\%) & & & & & \\
\hline Insulin alone & 30.4 & 10.6 & 35.4 & 25 & 100 \\
\hline Lifestyle & 39.1 & 84.8 & 29.9 & 0 & 0 \\
\hline OADS & 21.7 & 4.2 & 16.7 & 50 & 0 \\
\hline Insulin + OADs & 8.7 & 0.36 & 18.1 & 25 & 0
\end{tabular}

Conclusions: The minimum prevalence of MODY-type diabetes in children and adolescents is below $1 \%$ and our data give an up to date impression on frequency, phenotype and diabetes treatment of the most frequent MODYtypes. More prospectively collected phenotypes and systematic mutation screening will be required to give a true prevalence of different monogenic diabetes types.

\section{FC8-125 Diabetes and the Beta Cell}

\section{Urinary C-peptide creatinine ratio is a practical} outpatient tool in identifying MODY from Type 1 diabetes in children with diabetes

Rachel Besser ${ }^{1}$; Maggie Shepherd'; Timothy McDonald";

Beverley Shields'; Suzie Hammersley'; Bridget Knight'; Sian Ellard Andrew Hattersley ${ }^{1}$

${ }^{1}$ Peninsula Medical School, Peninsula NIHR Clinical Research Facility, Exeter, United Kingdom; ${ }^{2}$ Royal Devon and Exeter NHS Foundation Trust, Department of Clinical Biochemistry, Exeter, United Kingdom; ${ }^{3}$ Royal Devon and Exeter NHS Foundation Trust, Department of Molecular Genetics, Exeter, United Kingdom

Background: The young-onset hyperglycaemia seen in patients with maturity onset diabetes of the young (MODY) is frequently misdiagnosed as Type 1 diabetes (T1D), resulting in unnecessary treatment with insulin. Postprandial urinary C-peptide creatinine ratio (UCPCR) has been shown in adults to be a cheap, non-invasive test of endogenous insulin secretion and can discriminate MODY from long duration Type 1 diabetes $\geq 5$ years. The use of UCPCR in children close to diagnosis has not been established.

Objective and hypotheses: To assess whether UCPCR can discriminate MODY from Type 1 diabetes in children with short duration diabetes.

Methods: We measured 2 hour postprandial UCPCR in patients <18years with T1D $(n=87)$ and genetically confirmed MODY $(n=27 ;$ HNF1A/4A $n=9$, GCK $n=18$ ). Samples were collected at home and posted directly to the laboratory for analysis.

Results: T1D were similar to MODY patients in age (median(interquartile range, IQR) 13.1(10.5-15.4) v 13.0(11.6-16.0)y, $\mathrm{p}=0.34)$ and diabetes duration $(3.4(1.2-7.2)$ v $2.6(1.4-2.4) y, p=0.27)$. UCPCR was lower in T1D than MODY: (median (IQR) 0.07(<0.02-0.7) v $3.53(1.84-4.7) \mathrm{nmol} / \mathrm{mmol}$; $\mathrm{p}<0.0001)$. Receiver Operating Characteristic Curves showed excellent discrimination (area under curve 0.95 ) and identified a cut-off UCPCR $\geq 1.4 \mathrm{nmol} /$ mmol for discriminating MODY from T1D with $100 \%$ sensitivity and $85 \%$ specificity. $13 / 87$ (15\%) T1D patients with UCPCR $\geq 1.4 \mathrm{nmol} / \mathrm{mmol}$ were all diagnosed within 2.5 years of diagnosis.

Conclusions: A single post meal UCPCR can discriminate MODY from Type 1 in children with short duration diabetes. UCPCR may be a useful adjunct to select patients for diagnostic genetic testing.

\section{FC8-126 Diabetes and the Beta Cell}

\section{Reduced hippocampal volumes in children with congenital hyperinsulinism}

Anitha Kumaran'; ; Jemima Bullock'; Kling Chong ${ }^{3}$;

Faraneh Vargha-Khadem ${ }^{4}$; David Gadian ${ }^{5}$; Khalid Hussain ${ }^{1}$

'UCL Institute of Child Health and Great Ormond Street Hospital,

Clinical and Molecular Genetics Unit, London, United Kingdom;

${ }^{2}$ Great Ormond Street Hospital for Children NHS Trust, Department of Psychology, London, United Kingdom; ${ }^{3}$ Great Ormond Street Hospital for Children NHS Trust, Department of Radiology, London, United Kingdom; ${ }^{4} \mathrm{UCL}$ Institute of Child Health, Cognitive Neuroscience Unit, London, United Kingdom; ${ }^{5} \mathrm{UCL}$ Institute of Child Health, Radiology and Physics Unit, London, United Kingdom

Background: Bilateral parieto-occipital cortical and subcortical damage on magnetic resonance imaging (MRI) has been classically described in hypoglycaemic brain injury. The hippocampus plays an important role in the formation and consolidation of episodic memory/event memory, spatial navigation and awareness. Reduced hippocampal volumes accompanied by memory impairment and/or amnesia have been reported in conditions such as temporal lobe epilepsy, preterm children with low birth weight and those with cardiorespiratory disease.

Objective and hypotheses: To assess the integrity of the hippocampus in fifteen patients with congenital hyperinsulinism (CHI) who have been exposed to persistent recurrent hypoglycaemic episodes.

Methods: Fast low angle shot three-dimensional MRI scans were acquired on a 1.5T Siemens Avanto scanner. Neuroanatomical atlases were used to define borders of hippocampus bilaterally and volumes were calculated based on manual tracings of the regions. The hippocampal volumes then were corrected for intracranial volume.

Results: 15 children, 12 male and 3 females, age range 6-18 yrs were assessed. Three male children aged 6, 7.4 and 7.9 yrs showed bilateral reduction in hippocampal volume of more than $20 \%$ compared to 65 control subjects where mean right hippocampal volume was $3315 \pm 300 \mathrm{~mm} 3$ and left hippocampal volume $3206 \pm 268 \mathrm{~mm} 3$.The mean hippocampal volume of the 3 affected patients were $2028.86 \mathrm{~mm} 3,2153.5 \mathrm{~mm} 3$ and $2019.88 \mathrm{~mm} 3$ respectively.

Conclusions: This is the first study to assess the impact of CHI on hippocampal volumes. This study suggests that the hippocampus is highly susceptible to hypoglycaemic brain injury in children with CHI. The selective vulnerability of the hippocampus could be secondary to the abundance of NMDA (N-methyl D-aspartate) receptors that predispose to hypoglycaemia induced excitotoxic amino acid neuronal injury. Hippocampal volume reduction can impair episodic memory and have a huge impact on learning and daily functioning in these children. 


\section{FC8-127 Diabetes and the Beta Cell}

\section{How do the patients with transient neonatal} diabetes recur during long term follow-up?

Kanetee Busiah ${ }^{1}$; Pierre-François Souchon'; Claire Le Tallec ${ }^{3}$; Paul Czernichow'; Anne Paoli5; Raphaël Scharfmann ${ }^{6}$;

Bénédicte Gérard ${ }^{7}$; Hélène Cave; ${ }^{8}$ Michel Polak ${ }^{9}$

'Université Paris Descartes, INSERM U845, Faculté de Médecine

Necker-Enfants Malades, Paris, France; ${ }^{2}$ Amercican Memorial Hospital, CHU Reims, Pediatric Unit A, Reims, France; ${ }^{3}$ Hôpital des Enfants, Pediatric Unit, Toulouse, France; ${ }^{4}$ Necker Enfants Malades Hospital, APHP, Pediatric Endocrine Unit, Paris, France; ${ }^{5}$ Robert Debré Hospital, Diabetology and Endocrinology Unit, Reims, France; ${ }^{6}$ Université Paris Descartes, INSERM U845, Paris, France; ${ }^{7}$ Robert Debré Hospital, APHP, Genetic Biochemistry Unit, Paris, France; ${ }^{8}$ Robert Debré Hospital, INSERM U845, Paris, France; ${ }^{9}$ Pediatric Endocrine Unit, Necker Enfants Malades hospital, APHP, Université Paris Descartes, INSERM U845, Paris, France

Background: Transient neonatal diabetes mellitus (TNDM) is due to a severe non autoimmune insulin deficiency that occurs early in life and resolves in early childhood. About 50 to $70 \%$ of patients relapse during adolescence or adulthood. Very few data describe the relapses of the diabetes.

Objective and hypotheses: To investigate long term outcome of patients with TNDM and better characterize the recurrences.

Methods: The patients originated from the French Neonatal Diabetes Study Group. A total of 98 patients from 89 families had diabetes in the first 12 months of life, without beta-cell autoimmunity and no pancreas malformation and needed transient insulin treatment. We selected the patients who relapsed. Results: Twenty four individuals from 23 families (7 males, 17 females) had diabetes mellitus recurrence. Molecular aetiologies were mutation in ABCC8 $(n=6)$, KCNJ11 $(n=3)$, Insulin $(n=1)$ genes and 6q24 abnormality $(n=8)$. Among the latter group, 1 male and 1 female from the same family relapsed at 18 and 15,5 years respectively. A molecular etiology was not found in 6 patients. The median age at recurrence of 22 index cases was $12,8[1,8-$ 19] years; 21/22 (95\%) patients relapsed after 8 years of age. Relapse rate was $77 \%$ (23/30) with a minimal follow up of 8 years. Mode of recurrence, detailed for 16 children, was polyuria-polydipsia $(n=4)$ and hyperglycaemia without ketoacidosis ( $\mathrm{n}=12,3$ patients during an infectious disease). In this group, 1 patient alternated hyper and hypoglycaemia. Long term and repetitive metabolic tests (OGTT +/- IVGTT) in TNDM children up to at least 14 years old showed 3 different profiles: diabetic recurrence, glucose intolerance both with documented progressive loss of beta cell secretion and normal glucose metabolism.

Conclusions: In the population still followed up, relapse rate of TNDM is high. These results underscore the importance of performing a complete clinical and biological evaluation throughout infancy. Further studies are important to better characterize patients metabolic features before and after recurrences.

\section{FC8-128 Diabetes and the Beta Cell}

\section{Day-and-night closed-loop glucose control in adolescents with type 1 diabetes}

Daniela Elleri'; Janet Allen'; Kavita Kumareswaran ${ }^{2}$;

Lalantha Leelarathna'; Marianna Nodale²; Karen Caldwell;

Helen Murphy²; Malgorzata Wilinska'; Carlo Acerini; David Dunger ${ }^{1}$;

Roman Hovorka

'University of Cambridge, Department of Paediatrics and Institute of Metabolic Science, Cambridge, United Kingdom; ${ }^{2}$ University of Cambridge, Institute of Metabolic Science, Cambridge, United Kingdom; ${ }^{3}$ University of Cambridge, Department of Paediatrics, Cambridge, United Kingdom

Background: Tight glucose control is difficult to achieve in adolescents with type 1 diabetes (T1D).

Objective and hypotheses: To compare closed-loop (CL) insulin delivery with conventional pump therapy over 36h-period replicating common daily activity.

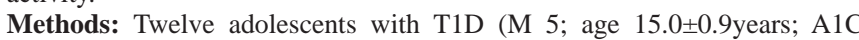
$7.9 \pm 0.7 \%$; BMI $21.4 \pm 2.6 \mathrm{~kg} / \mathrm{m} 2$; duration of diabetes $6.1 \pm 2.8$ years; total daily dose $1.0 \pm 0.4 \mathrm{U} / \mathrm{kg} /$ day; mean $\pm \mathrm{SD}$ ) were studied at a clinical research facility on two occasions. Subjects were randomly allocated to receive either CL or open-loop (OL) (conventional pump therapy) from 19:30 on day 1 to 08:00 on day 3. During CL, insulin pump basal rates were manually adjusted every $15 \mathrm{~min}$ as per advice of a model-predictive-control algorithm informed by real-time continuous glucose monitor. During control nights, conventional insulin pump settings were applied. On each occasion, subjects engaged in normal daily activities (e.g. playing computer games, walks) and consumed meals (50-80gCHO), accompanied by insulin boluses, and snacks (15-30gCHO). Moderate-intensity exercise on a stationary bicycle at $140 \mathrm{bpm}$ heart-rate was performed at 10:40 (40 min) and at 17:30 (20min) on day 2 .

Results: Overall mean plasma glucose levels were $7.2 \pm 1.2 \mathrm{mmol} / \mathrm{l}$ during CL versus $9.0 \pm 2.9 \mathrm{mmol} / \mathrm{l}$ during $\mathrm{OL}(\mathrm{p}=0.009)$. Time spent in target glucose range $3.9-10 \mathrm{mmol} / \mathrm{l}$ was $85(72,90) \%$ vs $56(26,81) \%$; median(IQR) $(\mathrm{p}=0.008)$. Time above $10 \mathrm{mmol} / \mathrm{l}$ was $9(4,21) \%$ vs $35(3,70) \%(\mathrm{p}=0.023)$ and time spent below $3.9 \mathrm{mmol} / \mathrm{l}$ was $4(2,10) \%$ vs $3(0,12) \%(\mathrm{p}=0.722)$. Overnight, plasma glucose levels were in target for $100(96,100) \%$ vs $56(6,90) \%$ $(\mathrm{p}=0.006)$ and for $100(98,100) \%$ vs $42(0,88) \%(\mathrm{p}=0.005)$ during the first and second night, respectively. Hypoglycaemia occurred on 11 occasions during OL vs 9 during CL (5 episodes were exercise-related, 4 occurred within 2 hours after meals).

Conclusions: Day-and-night CL may improve significantly glucose control compared to conventional pump therapy. Further research is needed to optimise insulin delivery to minimise risk of hypoglycaemia particularly around exercise.

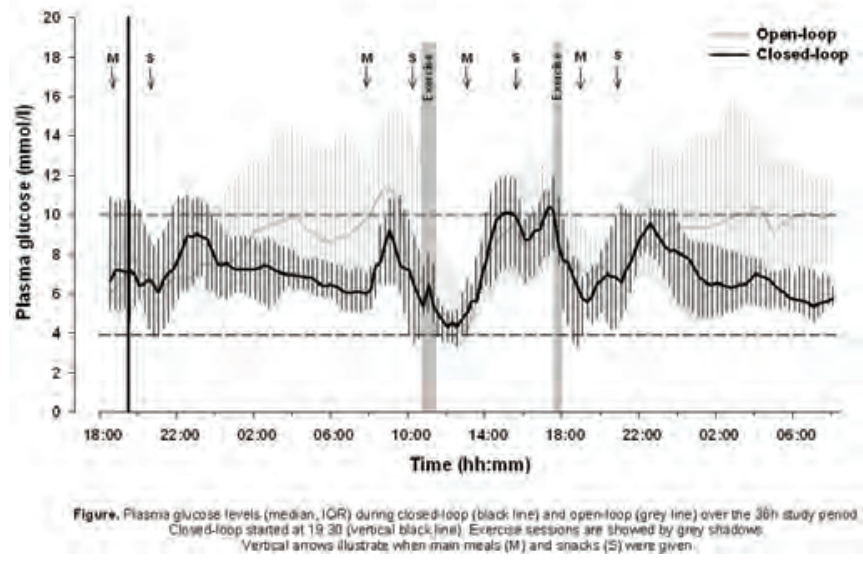

$\overline{\text { FC9-129 Reproductive System }}$

\section{PAX8: a new candidate gene for the} Mayer-Rokitansky-Kuster-Hauser syndrome? Mireille Castanet' ${ }^{1}$; Maud Bidet²; Raphaël Tessier ${ }^{3}$; Aurore Carré4; Elodie Tron ${ }^{4}$ Myriam Pépin-Donat ${ }^{5}$ : Michel Polak

${ }^{1} \mathrm{CHU}$ Charles Nicolle, Pediatrics Department, Rouen, France; ${ }^{2}$ Necker Enfant Malade Hospital, Pediatric Endocrinology Department, Paris, France; ${ }^{3} \mathrm{CHRU}$ Morvan, Pediatrics Department, Brest, France; ${ }^{4}$ Necker's Faculty, INSERM U845, Paris, France; ${ }^{5} \mathrm{CHU}$ Tours, Pediatrics Department, Tours, France

Background: Familial aggregation of MRKH syndrome suggests genetic causes but so far only few Wnt 4 mutations have been reported in affected patients and etiology remains unknown. Pax8-/- females mice reveal infertility due to müllerian duct agenesis suggesting that Pax8 could play a role in the uterine development. However, in humans, PAX8 mutations have been so far identified in patients affected by congenital hypothyroidism $(\mathrm{CH})$ with or without kidney agenesis and no associated uterine abnormalities have been reported.

Aim of the study: In one family including 7 members through 3 generations affected by $\mathrm{CH}$ due to either athyreosis, hemithyroid, ectopic or normal sized and sited thyroid gland, 2 girls suffered from additional primary amenorrhea, due to uterus agenesis. We thus hypothesized that Pax8 could be responsible for such association.

Methods: Direct sequencing of the binding domain of Pax8 (exons 2 to 4) was performed in all the $\mathrm{CH}$ members to search for Pax8 mutations.

Results: A new Pax8 heterozygous mutations (c320 334dup15) located within the binding domain was identified in all of the $\overline{C H}$ patients. Sequencing of the healthy relatives showed normal sequence. Abdominal ultrasound confirmed the uterus agenesis in 2 out of the 4 affected girls and no additional 
kidney malformations were found in the affected subjects.

Conclusion: Our data confirm the role of PAX8 in thyroid development either in proliferation and migration of the gland and strongly suggests that PAX8 could be regarded as a new candidate gene for the MRKH syndrome in humans. However, further genetic research in larger MRKH populations is needed to confirm this hypothesis.

\section{FC9-130 Reproductive System \\ Hypogonadism and testicular degeneration in Mulibrey nanism}

Susann Karlberg'; Jorma Toppari'; Niklas Karlberg'; Mirja Nurmio²; Riitta Karikoski3; Hannu Jalanko4; Marita Lipsanen-Nyman ${ }^{1}$

${ }^{1}$ Children's Hospital, University of Helsinki, Department of Paediatric Endocrinology, Helsinki, Finland; '2University of Turku, Departments of Physiology and Paediatrics, Turku, Finland; ${ }^{3}$ Helsinki University Central Hospital, Department of Pathology, Helsinki, Finland; ${ }^{4}$ Children's Hospital, University of Helsinki, Department of Paediatrics, Helsinki, Finland

Background: Mulibrey nanism (MUL) is a monogenic growth disorder with dysmorphic features, cardiopathy, and benign and malignant tumours. MUL is caused by mutations in the TRIM37 gene encoding TRIM37 protein possessing E3 ubiquitin-ligase activity.

Objective and hypotheses: As female MUL patients are infertile and develop premature ovarian failure before age 30 , we analyzed pubertal development, gonadal function and fertility in 28 Finnish male MUL patients (aged 2.2-43.2 years).

Methods: Clinical characteristics, hormone assays, semen analysis and testicular histology were assessed. Histological analyses were performed on testicular specimens from 8 patients and 4 controls obtained from biopsy, surgery or autopsy.

Results: The external genital phenotype was normal. In childhood and prepuberty, serum levels of FSH, LH, testosterone and inhibin B were normal. Puberty started spontaneously at a median age of 12.6 years (range 11.1-15.0). Until midpuberty, FSH, LH, testosterone and inhibin B levels increased adequately. Thereafter, testicular growth and virilization proceeded slowly. Concomitantly FSH, and to a lesser extent LH, showed a progressive increase to hypergonadotropic levels in all patients while inhibin B decreased and testosterone leveled off. Testicular size was small (mean volume 9.3ml, range 3.5$18.3 \mathrm{ml}$ in adults). All semen samples showed severe oligoasthenozoospermia $(n=13)$ or azoospermia $(n=4)$. None had a history of spontaneous fertility but four men had undergone infertility treatment and in one case the treatment (intracytoplasmic sperm injection, ICSI) was successful. All histological MUL samples showed varying degrees of degeneration.

Conclusions: All adult MUL males have a unique disorder of testicular function with small testes, elevated FSH and LH and low inhibin B. In MUL, mutations in TRIM37 lead to disturbance of sexual maturation in both sexes and fertility is severely compromised. Our findings suggest a role for TRIM37 in cellular functions regulating gametogenesis and gonadal function.

\section{FC9-131 Reproductive System}

\section{A novel c-terminal FSH $\beta$ mutation cause primary amenorrhea in three siblings}

Abdulsalam Abu-Libdeh'; Bassam Abu-Libdeh ${ }^{1}$; David Zangen ${ }^{2}$

${ }^{1}$ Makassed Islamic Hospital, Department of Pediatrics, Jerusalem,

Israel; ${ }^{2}$ Hadassah Hebrew University Medical Center, Division of

Pediatric Endocrinology, Department of Pediatrics, Jerusalem, Israel

Background: Inactivating mutations of the FSH $\beta$ subunit gene,causing isolated FSH deficiency and hypogonadism are very rare. To date, only six women with delayed puberty and isolated FSH deficiency have been reported worldwide.

Objective: Clinical and molecular studies were performed in three Palestinian sisters with impaired pubertal development and primary amenorrhea.

Methods: A 15y old female presented with delayed puberty, primary amenorrhea, and undetected basal serum FSH.LH peak during LHRH test was extremely high at $135 \mathrm{mIU} / \mathrm{ml}$ while FSH remained low at $<0.1 \mathrm{mIU} / \mathrm{ml}$. Abdominal sonography showed an infantile uterus. Two additional sisters presented later with a similar phenotype. Full pubertal development and menarche were easily achieved by estrogen and later progesterone replacement therapy. DNA was extracted from peripheral leukocytes and sequenced FSH beta gene.
Results: A homozygous 1bp frameshift deletion mutation in exon3 (354delGfs9) of the FSH $\beta$ gene was found in all three affected patients predicting an alteration of the 9 amino acids following codon 118 and a premature stop codon at position 127 . The parents and two healthy sisters were heterozygous. Interestingly ovarian biopsy revealed ovarian tissue with primordial follicles but no cysts and no leuteinized follicles and an adjacent sex cord ovarian stromal tumor with spindle cells and giant cells with eosinophilic granular cytoplasm, enlarged bizarre and hyperchromatic nuclei.

Conclusions: A novel FSH $\beta$ mutation has been detected in three hypogonadal sisters. This is only the fifth FSH $\beta$ mutation reported world wide and the first mutation not involving the cystine knot and the three $\beta$ hairpins of the protein that are essential to hormone binding. Further studies are required to determine the crucial role of the c-terminal 11 amino acids of FSH $\beta$ on the function of FSH given the severe clinical phenotype observed in our patients. The relation between the unique pathological phenotype observed here of sex cord - stromal tumor of ovary and the lack of FSH activity in the ovary should be further studied.

\section{FC9-132 Reproductive System \\ Insulin-like peptide 3 levels in boys with familial male-limited precocious puberty reflects premature activation of the LH-INSL3 signalling pathway \\ Najiba Lahlou'; ; Leandro Soriano-Guillen'2; Jonathan Oliel'; Yvette Le Bihan'; Marc Roger'; Jean-Claude Care ${ }^{\beta}$ \\ ${ }^{1}$ Hopital Cochin, Hormone Biology, Paris, France; ${ }^{2}$ Hospital Fundacion Jimenez Diaz, Paediatric Endocrinology, Madrid, Spain; ${ }^{3}$ Hopital \\ Robert-Debré, Paediatric Endocrinology, Paris, France}

Background: Insulin-Like Peptide 3 (INSL3) is a dimeric protein originating, like insulin, from a single-chain precursor. In the male it is secreted by Leydig cells under LH control and is involved in testicular descent during fetal development. Its relationship with LH is not an acute regulation and INSL3 level is considered as an index of the proliferative effect of LH.

Objectives: INSL3 secretion was investigated in boys bearing activating mutations of the LH receptor gene responsible for testotoxicosis or familial malelimited precocious puberty (FMPP), a dominant autosomal genetic disease clinically expressed only in males.

Population: Five boys from three families (mutation M398T or I542L) were investigated at clinical onset (at 2 to 6 years of age), then on testosterone supressive treatment by ketoconazole, and thereafter at adolescence when on LH suppressive treatment by GnRH agonist after initiation of central puberty. Gonadotropin, testosterone and INSL3 levels were assessed by specific immunoassays. The Mann-Whitney test was used for comparison between groups.

Results: INSL3 levels in boys with FMPP at 6 to 10 years of age were significantly higher than in matched controls: mean and range 277 (37-638) vs 128 (38-382) $\mathrm{pg} / \mathrm{ml}$, respectively, $(\mathrm{p}=0.016)$. After pubertal onset, when treated with a GnRH agonist, INSL3 levels further increased despite LH suppression: mean and range 747 (146-2046) $\mathrm{pg} / \mathrm{ml}$ at 12-17 years of age $(\mathrm{p}=0.0002$ relative to $6-10$ years).

Conclusion: In boys with activating mutation in the LH receptor gene, despite low LH levels, INSL3 secretion was significantly increased at prepubertal age. That gives evidence of the stimulatory effect of the LH signalling pathway activation on INSL3 expression. After initiation of puberty, and achievement of pubertal LH levels, INSL3 secretion, as expected, further increased, which likely denotes a significant proliferative effect of LH. 


\section{FC9-133 Reproductive System}

Longitudinal serum levels of Anti-Müllerian Hormone in $\mathbf{8 5}$ healthy girls compared to FSH, LH, inhibin B, estradiol, testosterone, SHBG before and after pubertal onset

Casper P. Hagen; Lise Aksglæde; Kaspar Sorensen; Annette Mouritsen; Anne-Maria Andersson; Anders Juul Rigshospitalet, University of Copenhagen, Department of Growth and Reproduction, Copenhagen, Denmark

Background: In adult women, circulating level of Anti Müllerian Hormone (AMH) is thought to predict the number of remaining ovarian follicles. During the postnatal gonadotropin surge, the AMH level increases. Little is known about AMH regulation and function in postnatal life, but AMH may preserve the follicle pool.

Objective and hypotheses: To study AMH level longitudinally in girls through childhood and adolescence and the relation to other reproductive hormone. To determine if the individual AMH level varies during puberty.

Methods: 85 healthy girls and adolescents were examined biannually in the COPENHAGEN Puberty Study. 39 girls entered puberty during follow-up. We measured AMH, FSH, LH, inhibin B, estradiol, testosterone and SHBG according to age and pubertal stage. The total number of blood samples was 508 (median 6 per girl; range 2-10).

Results: The individual mean AMH ranged from 5-54 pmol/L (median 18). The mean intra-individual coefficient of variation (CV) of AMH was $22.0 \%$ (range $0.1-53.7 \%$ ), which exceeded the AMH inter-assay CV (10.8\%) in $71 / 85$ girls $(84 \%)$. Based on a variance components model, AMH decreased $30 \%$ during the first 2 years after pubertal onset (from 24 to $17 \mathrm{pmol} / \mathrm{L}$, $\mathrm{p}<0.001$ ). The relative decline was independent of the AMH level. Subsequently, AMH levels were constant. There was no significant correlation between AMH (mean level prior to pubertal onset) and age of pubertal onset. During mid-childhood, there was a clear negative association between AMH and both FSH and LH levels. No associations to other reproductive hormone levels were found.

Conclusions: A random AMH value indicates a permanent level for any given girl during childhood. Although AMH declines after pubertal onset, AMH is remarkably constant compared to other reproductive hormones during puberty. Interestingly, AMH is inversely associated with gonadotropin-levels even in pre-pubertal girls, indicating that AMH is a marker of ovarian activity during childhood.

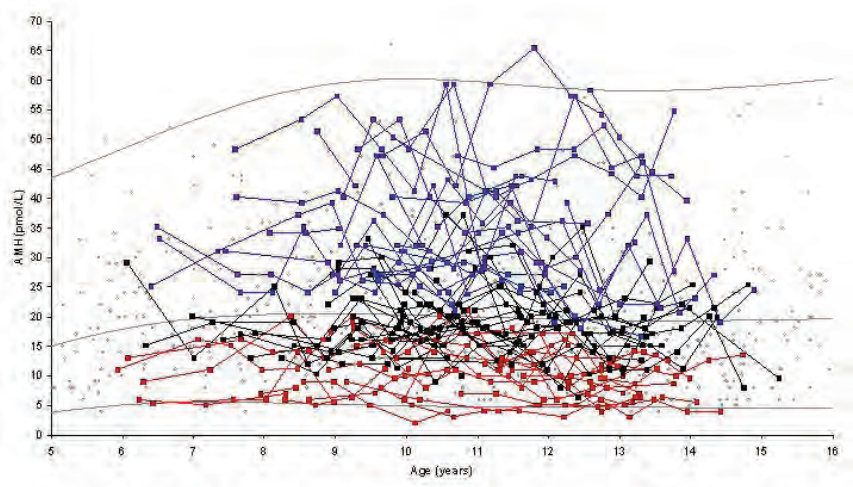

Longitudinal levels of AMH in 85 healthy girls (number of samples: total, median, range: $508,6,2-10$ ) compared to a reference range based on 926 healthy Danish fermales (grey dots). Thin grey lines represen median, the 2.5n percentle and the $97.5^{\text {th }}$ percentile. Girls with the hignest

\section{FC9-134 Reproductive System}

Towards early metformin therapy (age 8-12 yr) to prevent Polycystic Ovary Syndrome (PCOS) in adolescence

Lourdes Ibañez'; Abel Lopez-Bermejo²; Marta Díaz;

Maria Victoria Marcos ${ }^{4}$; Francis de Zegher ${ }^{5}$

${ }^{1}$ Hospital Sant Joan de Déu, University of Barcelona, Endocrinology, Esplugues, Barcelona, Spain; ${ }^{2}$ Hospital Dr. Josep Trueta, Pediatrics, Girona, Spain; ${ }^{3}$ Hospital Sant Joan de Déu, Endocrinology, Esplugues, Barcelona, Spain; ${ }^{4}$ Hospital de Terrassa, Endocrinology, Terrassa, Barcelona, Spain; ${ }^{5}$ University of Leuven, Woman \& Child, Leuven, Belgium

Context: Girls with a combined history of low(-normal) birthweight (LBW) and precocious pubarche (PP) are at high risk to develop PCOS.

Objective: To compare the capacity of early versus late metformin treatment to prevent adolescent PCOS.

Design: Randomized, open-label study over 7 yr.

Setting: University Hospital.

Patients: 38 LBW-PP girls followed from mean age 8 till $15 \mathrm{yr}$.

Intervention: Early metformin (study year 1-4; age 8-12 yr) versus late metformin (year 5; age 13-14 yr).

Main outcome measures: Height; weight; endocrine-metabolic screening (fasting; follicular phase); C-Reactive Protein (CRP); body composition (absorptiometry); abdominal fat partitioning (MRI); ovarian morphology (ultrasound); PCOS (NIH \& AES definitions) after year 7 (all girls thus untreated for at least $1 \mathrm{yr}$ ).

Results: None of the girls dropped out of the study. At age 15 yr, early-metformin girls were taller $(4 \mathrm{~cm})$, were in a less pro-inflammatory state, and had a less central fat distribution due to reductions of visceral and hepatic fat. PCOS was 7- to 10-fold more prevalent in late- than early-treated girls. Abdominal adiposity was the first variable to diverge (at age 8-10 yr) between girls without versus with PCOS at age $15 \mathrm{yr}$, irrespective of metformin treatment.

Conclusions: In LBW-PP girls, early metformin therapy was found to prevent or delay the development of PCOS more effectively than late metformin. The time window of late childhood and early puberty may be more critical for the development - and thus for the prevention - of adolescent PCOS than the first years beyond menarche. This principle may also apply to other/obese girls at risk of adolescent PCOS.

\section{FC10-135 The X Chromosome \\ Is the region Xp11.4-Xp22.1 harboring the presumptive second short stature gene in Turner syndrome?}

Antonis Voutetakis ${ }^{1}$; Amalia Sertedaki ${ }^{1}$; Maria Dracopoulou', Kostas Deligeoroglou'; Markos Mihalatos ${ }^{3}$; Harry Kontos 3 ;

Catherine Dacou-Voutetakis ${ }^{1}$

1 University of Athens, Medical School, First Department of Pediatrics, Division of Endocrinology, Diabetes and Metabolism, Athens, Greece; 2University of Athens, Medical School, Department of Obstetrics and Gynecology, Athens, Greece; ${ }^{3}$ Genomedica, Molecular Diagnostics Laboratory, Athens, Greece

Background: Short stature is a cardinal and most frequently encountered sign in girls with Turner syndrome (TS). Thus far, only one gene, (ie SHOX), the PAR 1 stature gene related to short stature in this syndrome, has been identified. SHOX haploinsufficiency by itself accounts for an estimated mean height deficit of about -2 SD. By contrast, the growth deficiency associated with large Xp deletions is similar to that of complete monosomy X (-3.2 SD). It has thus been hypothesized that the difference in mean height deficit of small versus large deletions might be due to the existence of a second $\mathrm{Xp}$ stature locus in the interval Xp11.2-Xp22.1.

Objective and hypotheses: To study a mother and daughter as unique paradigms of Xp deletion, testing the hypothesis that this deletion might harbor the presumptive second short stature TS gene.

Methods: We report a mother and daughter with short stature (height SDS -3.4) and no other pathologic characteristics: normal puberty, menses, gonadotropins fertility and absence of thyroid autoimmunity. Karyotype of peripheral lymphocytes and array CGH analysis were carried out by appropriate methodology. 
Results: The regular Karyotype in mother and daughter disclosed an Xp deletion (Xp 22.1 to Xp21.1). Further study with array CGH analysis revealed a deletion encompassing about 13.37 MB from Xp 11.4 to Xp 22.11. Although the SHOX gene lies outside this deletion, confirmation of the presence of SHOX was sought through the use of 4 probes: RP13-465B17, RP13-391G2, RP13-76L22, RP13-167H21.

Conclusions: The present data support the existence of a second short stature gene related to the growth deficit in TS and located in the Xp 11.4-Xp 22.1 region.

\section{FC10-136 The X Chromosome}

The parental origin of the $X$ chromosome does not affect the effect of growth hormone and psychosocial components in Turner syndrome Marie Devernay'; Diana Bolca²; Bénédicte Gérard ${ }^{13}$;

Anne-Claude Tabet'; Brigitte Benzacken ${ }^{4}$; Emmanuel Ecosse ${ }^{5}$; Jean-Claude Care/ ${ }^{6}$

${ }^{1}$ Robert Debré Hospital, Pediatric Endocrinology and Diabetology, INSERM U690, Paris, France; ${ }^{2}$ Robert Debré Hospital, Pediatric Endocrinology and Diabetology, Paris, France; ${ }^{3}$ Robert Debré Hospital, Genetic Biochemistry, Paris, France; ${ }^{4}$ Robert Debré Hospital, Genetics, Paris, France; ${ }^{5}$ Groupe Hospitalier Cochin-Saint Vincent de Paul, Biostatistics, Paris, France; ${ }^{6}$ Robert Debré Hospital, Pediatric Endocrinology and Diabetology, INSERM U690, University Paris 7 Denis Diderot, Paris, France

Background: The phenotype in Turner syndrome (TS) is variable in patients with nonmosaic 45,X karyotype. Previous studies have identified the parental origin of the $\mathrm{X}$ chromosome as influencing several aspects of TS, in particular the response to growth hormone (GH).

Objective: We evaluated the parent-of-origin effect of the X chromosome on growth and psychosocial characteristics of women with TS in a large cohort with nonmosaic 45,X karyotype.

Methods: A national cohort (Statur-X cohort) of 556 young women with TS, aged $26.1 \pm 4.2 \mathrm{yr}$ (18.1-35.8). Parental origin of the X-chromosome determined by comparing patient and parental microsatellite markers. Association between the parental origin of the $\mathrm{X}$ chromosome and characteristics of TS women.

Results: 230 (49\%) patients and their mother participated and parental origin of the X chromosome was assigned in 185 . 57/185 women (31\%) had a paternal and 128/185 (69\%) a maternal chromosome X. Total gain in height from start of GH to adult height expressed in SDS for TS was similar in Xmat and Xpat groups $(2.0 \pm 0.9$ vs $2.2 \pm 0.8 \mathrm{p}=0.362)$. Similarly, there were no significant differences between group in parental heights, midparental height, birth weight, age, weight and height at GH initiation, duration of GH therapy, mean GH dose, first-year growth on GH, height at end of GH treatment, adult weight and body mass index. Using multivariate analysis, parental origin of $\mathrm{X}$ chromosome was similarly not associated with adult height gain after adjustment on baseline height. The Xpat group was slightly shorter at birth (46.1 \pm $3.1 \mathrm{~cm})$ than the Xmat group $(47 \pm 2.3 \mathrm{~cm}, \mathrm{p}=0.022)$ and born earlier $(38.7$ \pm 2.2 weeks of amenorrhea vs $39.4 \pm 1.3, \mathrm{p}=0.016$ ). Social adjustment and self-esteem were similar between the two groups (SEI scale, SAS-SR scale and GHQ-12 score).

Conclusion: In the largest study so far, the parental origin of the $\mathrm{X}$ chromosome did not seem to affect the response to GH, most auxological characteristics and the studied psychosocial components in TS. There was a modest potential effect on birth length and gestational age.

\section{FC10-137 The X Chromosome}

Correlation of mRNA expression profiles with clinical response to growth hormone in Turner syndrome

Adam Stevens ${ }^{1}$; Shahin Tajbakhsh'; Andrew Whatmore'; Melissa Westwood'; Peter Clayton

${ }^{1}$ Manchester University, Manchester Academic Health Sciences Centre, Manchester, United Kingdom; ' ${ }^{2}$ Manchester University, Maternal \& Fetal Health Research Centre, Manchester, United Kingdom

Background: Recombinant human growth hormone (rhGH) is used to improve final height in girls with Turner syndrome (TS) but the gain is variable $(0-20 \mathrm{~cm})$. Only $46 \%$ of variability in the first year response to rhGH can be accounted for by prediction models, implying genetic profiling data could improve prediction.

Objective and hypotheses: We have used an ex-vivo fibroblast model to study mRNA expression profiles and associated biological pathways in response to rhGH.

Methods: Three fibroblast cell lines were used, C10 - control; T1 - TS patient responding well to rhGH (height SDS change over 4 years of +1.8 ) and T5 - poor responder to rhGH (height SDS change over 4 years of -0.3 ). Gene expression was measured at 0 and $24 \mathrm{hr}$ after incubation with GH (200ng/ ml) using Affymetrix HG-U133 arrays and analysed using Ingenuity Pathway Analysis software (IPA). Changes in gene expression were confirmed by quantitative PCR.

Results: Variability in basal gene expression between C10 and TS cells (408 genes, $\mathrm{p}<0.05$ ) clustered in pathways associated with development of reproductive, cardiac, skeletal systems and muscular function (IPA, $\mathrm{p}<0.05$ ). Basal comparison of the T1 and T5 cell lines showed differentially expressed genes (92 genes, $\mathrm{p}<0.05$ ) associated with development, muscular function and cardiac development (IPA, $\mathrm{p}<0.05$ ). GH significantly modified the expression of 219 genes in T5, 67 in T1 and 79 in C10 $(\mathrm{p}<0.01)$. IPA identified $(\mathrm{p}<0.05)$ GH-sensitive pathways including cellular growth and proliferation (all 3 cell lines), calcium signalling pathways (T1) and amino acid metabolism pathways (T5). The genes most regulated by GH treatment (but unchanged in the other cell lines) were; IGFBP3 in T5 ( $<<0.009,-5.9$ fold), DEPDC1, a modulator of GTPase activity in T1 ( $<<0.01,1.6$ fold) and MMP3 in C10 $(\mathrm{p}<0.0004,20$ fold $)$.

Conclusions: This work has identified candidate biological pathways that differentiate growth response along with both recognised and novel biomarkers for clinical response to rhGH in Turner Syndrome.

\section{FC10-138 The X Chromosome \\ Identification of the first PAR1 deletion upstream of SHOX in a patient with idiopathic short stature (ISS)}

Sara Benito-Sanz'; Miriam Aza-Carmona'; Amaya Rodríguez-Estévez²; Itxaso Rica'; Ricardo Gracia ${ }^{3}$; Ángel Campos-Barros' ${ }^{1}$ Karen E. Heath ${ }^{1}$ ${ }^{1}$ Hospital Universitario La Paz, UAM, IdiPAZ; CIBERER, ISCIII, Institute of Medical and Molecular Genetics (INGEMM), Madrid, Spain; ${ }^{2}$ Hospital de Cruces; CIBERER, ISCIII, Pediatric Endocrinology, Barakaldo, Spain; ${ }^{3}$ Hospital Universitario La Paz, Pediatric Endocrinology, Madrid, Spain

Background: Alterations in SHOX and its downstream transcriptional regulatory elements, located in the pseudoautosomal region 1 (PAR1), are present in $\sim 60 \%$ and $~ 5 \%$ of patients with Léri-Weill dyschondrostosis and idiopathic short stature (ISS) respectively. The molecular defect is unknown in the remaining patients. In the last few years, six SHOX enhancers have been identified in the PAR1, three downstream and more recently, three upstream of SHOX. To date, no alteration of the upstream SHOX enhancer region has been observed in LWD or ISS cases.

Objective: To screen for alterations in the upstream SHOX transcription regulatory elements in LWD and ISS patients with no known molecular defect. Methods: A MLPA assay was designed to include the region upstream of SHOX consisting of 13 PAR1-specific probes, three of which are located within the upstream SHOX enhancers. Alterations were confirmed and delimited using array-CGH (Nimblegen).

Results: An upstream SHOX deletion was identified in one patient with ISS. The deletion extends between $337-250 \mathrm{~kb}$. The deletion does not include 
SHOX but includes two of the upstream SHOX enhancers. The patient, a girl of 13.5 years has proportional short stature $(140.3 \mathrm{~cm},<\mathrm{P} 3)$ with normal GH and IGF-I levels. Father's and mother's height are $163 \mathrm{~cm}(-1.76 \mathrm{SD})$ and 162 cm (-0.03 SD) respectively.

Conclusions: 1) We identified the first deletion that includes the upstream SHOX enhancer elements in a patient with ISS. The loss of these elements may result in a decrease in the transcription of SHOX. 2) Genetic screening of this region should be undertaken in all patients with a diagnosis of LWD and ISS. For this, additional probes of this region should be included in the commercial MLPA kit so that a more comprehensive analysis of deletions and duplications of PAR1 can be undertaken in one assay.

\section{FC10-139 The X Chromosome}

\section{Identification of a novel downstream SHOX} enhancer, deleted in patients with LWD and ISS Sara Benito-Sanz'; Jose Luis Royo²; Eva Barroso'; Ricardo Gracia3; Ángel Campos-Barros ${ }^{1}$; Jose Luis Gómez-Skarmeta ${ }^{2}$; Karen E. Heath ${ }^{1}$ ${ }^{1}$ Hospital Universitario La Paz, UAM, IdiPAZ; CIBERER, ISCIII, Institute of Medical and Molecular Genetics (INGEMM), Madrid, Spain; ${ }^{2}$ Centro Andaluz de Biología del Desarrollo, CSIC-Universidad Pablo de Olavide, Sevilla, Spain; ${ }^{3}$ Hospital Universitario La Paz, UAM, Pediatric Endocrinology, Madrid, Spain

Background: $S H O X$, located in the pseudoautosomal region 1 (PAR1), encodes a transcriptional factor implicated in human growth. A total of six enhancers have been identified in the upstream and downstream sequences. Deletions of SHOX or the downstream enhancer regions, SHOX duplications or point or indel mutations within SHOX have been observed in 60\% Leri-Weill dyschondrosteosis (LWD) and 5-15\% idiopathic short stature (ISS) patients. The molecular defect is unknown in the remaining patients.

Objective and hypotheses: To identify and characterize the molecular defect in the remaining $40 \%$ of LWD patients without a known pathogenic alteration.

Methods: 124 LWD/possible LWD and 576 ISS patients were screened or re-evaluated for PAR1 alterations by MLPA. Alterations were subsequently confirmed and delimited by specifically designed MLPAs, array-CGH (Nimblegen) and breakpoint junction PCR/sequencing. The evolutionarily conserved regions (ECRs) identified within the deleted region were functionally analyzed for $S H O X$ regulatory activity using: 1) Chromosome Conformation Capture (3C) in chicken limbs and 2) Luciferase assay in human U2OS cells. Results: A small downstream deletion downstream of $\mathrm{SHOX}$ was identified in 11 LWD and 17 ISS patients. The deletion extends 47544 bp and was identical in all patients. Seven ECRs lay within the deleted region. The $3 \mathrm{C}$ data indicated that one ECR, conserved from human to zebrafish, may be a SHOX regulatory region. Confirmation was obtained by expressing this ECR in human U2OS cells and assessing their enhancer capacity using a luciferase assay. Enhancer activity was observed for ECR1.

Conclusions: 1) We have identified and characterized a common $\sim 47.5 \mathrm{~kb}$ downstream PAR1 deletion in LWD and ISS patients; 2) A novel SHOX enhancer was identified within the deleted region; 3) We propose that the deletion of this enhancer may explain the pathogenic mechanism for their phenotype observed in these patients.

\section{$\overline{\text { FC10-140 The X Chromosome }}$}

\section{Spontaneous osteoclastogenesis TNF- $\alpha$ and RANKL mediated in patients with Turner syndrome \\ Maria Felicia Faienza ${ }^{1}$; Giacomina Brunetti2; Annamaria Ventura ${ }^{1}$;} Silvia Colucci ; Laura Piacente ${ }^{1}$; Maria Ciccarelli' ${ }^{\text {; }}$ Olinda D'Addato', Filomena Ladisa'; Angela Oranger²; Maria Grano²; Luciano Cavallo ${ }^{1}$ University of Bari, Biomedicine of Developmental Age, Bari, Italy; ¿University of Bari, Human Anatomy and Histology, Bari, Italy

Background: Reduced bone mineral density and increased fracture risk have been reported in Turner syndrome (TS). Although hypoestrogenism play a critical role in the alteration of bone mineralization in TS, other factors seems to be involved. Studies in mice suggest that high FSH levels can cause bone loss even if estrogen levels are relatively normal. A possible mechanism by which FSH decreases bone mass is by inducing TNF- $\alpha$ production in T cells TNF- $\alpha$ and RANKL are two cytokines involved in formation of osteoclasts (OCs).
Objective and hypotheses: The aim of this study was to investigate the osteoclastogenic potential of peripheral blood mononuclear cells (PBMCs) from 10 TS patients (mean age $10.44 \pm 5.48$ ), and from 10 controls (mean age $12 \pm$ 4.24). We also evaluated the role of $\mathrm{T}$ cells in OC formation.

Methods: PBMCs and T cell depleted culture from patients and controls were cultured in presence/absence of recombinant human macrophage -colony stimulating factor (M-CSF) (25 ng/ml) and RANKL (30 ng/ml). At the end of the culture period, mature multinucleated OCs were identified as tartrateresistant acid phosphatases positive (TRAP+) cells. By real-time PCR we studied TNF- $\alpha$ and RANKL expression in freshly isolated T cells.

Results: Spontaneous osteoclastogenesis, without adding M-CSF and RANKL, and significantly higher OC resorbing activity occurred in TS. Conversely, M-CSF and RANKL were essential to trigger and sustain osteoclastogenesis in PBMCs from controls. Additionally, we demonstrated a T celldependent osteoclastogenesis in TS PBMCs. T cells from patients showed high mRNA levels of both TNF- $\alpha$ and RANKL respect to controls, with a greater expression of the former.

Conclusion: The present study showed for the first time a high osteoclastogenic potential of PBMCs from TS patients, that seems supported by the osteoclastogenic factors TNF- $\alpha$ and RANKL released by T cells.

\section{FC11-141 Diabetes Complications}

\section{Adhesion molecules [intercellular adhesion molecule-1 (ICAM-1) and vascular cell adhesion molecule-1 (VCAM-1)], cyclooxygenase-2 (COX-2) and matrix metalloproteinase-9 (MMP-9) in type 1 diabetic children and young adults}

Nikolitsa Koutroumani' ; Andrea-Paola Rojas-GiP; Alexia Karvela', Athina Dettoraki'; Bessie E. Spiliotis ${ }^{1}$

${ }^{1}$ University of Patras, Research Laboratory of the Division of Pediatric Endocrinology and Diabetes, Department of Pediatrics and Diabetes, Patras, Greece; ' ${ }^{2}$ nniversity of Peloponnese, Faculty of Human Movement and Quality of Life Sciences, Department of Nursing, Sparta, Greece

Background: Advanced Glycation Endproducts(AGEs), via their receptor RAGE, induce the activation and migration of mononuclear cells in the subendothelium and play a major role in diabetic angiopathy. COX-2 produces prostaglandin PGE2 which increases the expression of matrix metalloproteinases (MMPs) inducing the formation of sRAGE, an endogenous inhibitor of RAGE signaling. ICAM-1 and VCAM-1 are adhesion molecules (activated through the AGEs-RAGE axis) that mediate leukocyte adhesion to the endothelium.

Objective and hypotheses: To study the levels of COX2, ICAM-1, VCAM-1 and MMP-9 expression in DMI children and young adults.

Methods: COX-2, MMP9, ICAM-1 and VCAM-1 gene expression (COX-2g, MMP-9g, ICAM-1g, VCAM-1g respectively) and COX-2 protein expression (COX-2pr) were measured by RT-PCR and Western Immunoblotting respectively, in isolated peripheral blood mononuclear cells from children 4-18yrs, (89 DMI,92 controls) and young adults, 18-29yrs, (20 DMI,19 controls). Serum MMP-9 levels (MMP-9lev) were determined by ELISA.

Results: COX-2pr was significantly decreased in DMI patients $>13$ years old and in those with HbA1c $>8 \%$ in comparison to their age matched controls. MMP-9 serum levels significantly decreased with $\mathrm{HbA} 1 \mathrm{c}>10 \%$ whereas MMP-9g showed no differences. VCAMg was significantly decreased in all DMI patients in comparison to their controls and ICAMg showed no differences with the controls.

Conclusions: The decrease observed in COX2pr in the DMI $>13$ years old and in those with HbA1c > $8 \%$ together with a decrease in MMP-9 with HgbA1C $>10 \%$ may show that these DMI individuals are at a greater risk of diabetic complications, in the long-term, if these parameters lead later to a reduction in sRAGE levels. In the short term though, these DMI children and young adults may be protected from these complications since VCAM is significantly decreased, possibly through an alternative protective pathway, and the expression of ICAM has not yet been affected. 


\section{FC11-142 Diabetes Complications}

Flow mediated dilatation and carotid intima media thickness, markers of atherosclerosis and cardiac dysfunction in type 1 diabetes? Damla Goksen'; Sakine Kar²; Erturk Levent ${ }^{3}$; Ozlem Korkmaz'; Sukran Darcan

${ }^{1}$ Ege University, Faculty of Medicine, Pediatric Endocrinology, Izmir, Turkey; ${ }^{2}$ Ege University, Faculty of Medicine, Pediatrics, Izmir, Turkey; ${ }^{3}$ Ege University, Faculty of Medicine, Pediatric Cardiology, Izmir, Turkey

Objectives and hypotheses: To examine the occurrence of subclinical markers of cardiovascular risk and cardiac dysfunction and their association with biochemical markers and metabolic control in Type 1 diabetes mellitus.

Methods: The study included 55 Type 1 diabetic patients (31F/24M) (17, $6 \pm 4$ years) without any cardiovascular abnormalities and 30 healthy aged matched subjects (14F/16 M) (16, 4 $\pm 4,1$ years) served as the control group. HbA1c, lipid profile, HsCRP and adiponectin were evaluated as the biochemical markers of arteriosclerosis and metabolic control. Standard echocardiography (2D, Doppler), myocardial performance index (MPI), Carotid intima media thickness (CIMT) and flow mediated dilatation (FMD) were performed.

Results: HsCRP levels in children and adolescents with diabetes were significantly elevated compared with the control group $(0,21 \pm 0,31$ vs. $0,1 \pm 0,16$ $\mathrm{mg} / \mathrm{l}$ p.0,00) whereas no significant difference was found in adiponectin concentrations between the two groups $(15,2 \pm 6,1$ vs $15,57 \pm 6,49 \mathrm{mcg} / \mathrm{ml})$. In MPI abnormalities and diastolic dysfunction of both ventricules were shown in Doppler measurements in diabetic patients (p:0,01) FMD measurements were decreased and CIMT was increased in the study group(p:0,001). Duration of diabetes correlated positively with CIMT and negatively with FMD. FMD corelated negatively with $\operatorname{HsCRP}(r=-0,28, p=0,03)$, and adinopectin ( $r=-0,27, p=0,04)$.

Conclusion: Increased HsCRP and CIMT, decreased FMD and diastolic dysfunction in the study group show that children with type 1 diabetes have cardiaovascular disease risk. Standard echocardiographic measurements were independent from duration of diabetes, insulin dose and metabolic control whereas advanced techniques as FMD and CMIT were influenced by duration of diabetes. As a result this study shows the necessity of advanced techniques as MPI, CIMT and FMD in the follow up of type 1 diabetes.

\section{FC11-143 Diabetes Complications \\ The effect of visceral adiposity, blood pressure, HbA1c, and LDL-cholesterol on the longitudinal evolution of the carotid intima medial thickness in children with type 1 diabetes mellitus. Longitudinal cIMT development in T1DM}

Robert Dalla Pozza'; Claude Thilmany'; Claudia Weissenbacher; Heinrich Netz'; Heinrich Schmidt ${ }^{2}$; Susanne Bechtold ${ }^{2}$ 'University Children's Hospital, Pediatric Cardiology, Munich, Germany; 'University Children's Hospital, Pediatric Endocrinology and Diabetology, Munich, Germany

Background: Type 1 diabetes mellitus is a generally accepted atherogenic risk factor.

Objectives: The aim of this prospective longitudinal study was to evaluate changes in carotid intima media thickness (cIMT) in children and adolescents with type 1 diabetes mellitus (T1DM) using standardized methods.

Method: We re-evaluated cIMT in 70 (38 f) of initial 150 (80 f) patients with T1DM after 4 years. At re-evaluation mean age was $16.45 \pm 2.59 \mathrm{y}$, mean diabetes duration was $9.2 \pm 3.24 \mathrm{y}$ and patients had a mean HbA1c of 8.14 $\pm 1.06 \%$.

Results: Mean cIMT z-scores increased significantly during 4 years $(0.58 \pm$ 0.75, $\mathrm{p}<0.001) .46 / 70$ patients had an increase in cIMT; compared to the 24 pts. with a cIMT stabilization these pts. were older $(p=0.011)$, more advanced in pubertal development $(\mathrm{p}<0.001)$, and had a higher systolic blood pressure $(\mathrm{p}=0.031)$. Waist-to-hip ratio was significantly correlated to cIMT values $(\mathrm{r}=0.29 ; \mathrm{p}=0.049)$. Moreover, in a multivariate regression model (backward) for cIMT increment significant associations $(\mathrm{R} 2=0.505, \mathrm{p}=0.022)$ were systolic blood pressure $(\beta=0.301, p=0.053)$, LDL-cholesterol $(\beta=0.238$, $\mathrm{p}=0.086)$, HbA1c $(ß-0.409, \mathrm{p}=0.021)$ and WHR $\mathrm{z}$-score $(ß=0.274, \mathrm{p}=0.01)$. Conclusion: Longitudinal cIMT measurements revealed progression in sub- clinical atherosclerosis during a four year period in diabetic children and adolescents. Classical cardiovascular risk factors were related to cIMT increment. Control of these risk factors by lifestyle and medical intervention may prevent progression of cIMT in diabetic children.

\section{FC11-144 Diabetes Complications \\ Can neutrophil gelatinase-associated lipocalin be an early marker of kidney injury in type 1 diabetics? \\ Korcan Demir'; Ayhan Abaci'; Tuncay Kume²; Ayca Altincik'; Gonul Catli'; Ece Bober ${ }^{1}$ \\ 'Dokuz Eylul University, Department of Pediatric Endocrinology, Izmir, Turkey; ' ${ }^{2}$ okuz Eylul University, Department of Biochemistry, Izmir, Turkey}

Background: Biomarkers other than microalbuminuria are needed to detect early kidney injury in children with type 1 diabetes.

Objective and hypotheses: To determine the place of neutrophil-gelatinase associated lipocalin (NGAL) in detecting diabetic kidney injury in normoalbuminuric and normotensive adolescents with type 1 diabetes and to assess the relationship between NGAL and clinical and laboratory variables.

Methods: Forty six consecutive adolescents with type 1 diabetes [M/F: 24/22; median age 14.5 (12.2-16), diabetes duration 4.8 years (2.6-6.7), hemoglobin A1c 7.9\% (7.2-9.2)] and 21 control cases [M/F: 7/14; median age 14.8 (13.615.5)] were compared regarding clinical, laboratory (including urine and plasma NGAL), and ambulatory blood pressure monitoring (ABPM) variables. According to risk factors including high-normal albuminuria, long-term hemoglobin $\mathrm{A} 1 \mathrm{c}>8.2 \%$, diabetes duration $\geq 5$ years, and hypertension detected by ABPM, type 1 diabetics were divided into subgroups allowing further assessment of association of NGAL levels with study variables.

Results: Median blood and urine glucose, hemoglobin A1c, urinary NGAL/ creatinine ratio [13.2 (8.3-43.1) vs. $4.8(2.9-20.2), \mathrm{p}=0.015]$, daytime systolic and diastolic blood pressure (BP) SD score, and daytime systolic and diastolic BP loads were found higher in diabetics. Urine NGAL levels were correlated with albumin/creatinine ratio $(r=0.452, p=0.002)$ while plasma NGAL levels were correlated with nighttime systolic BP load $(r=0.309, p=0.037)$. Subgroup analyses showed similar NGAL levels except higher median urine NGAL levels [48.7 ng/mL (27.9-149.1) vs. $11.2 \mathrm{ng} / \mathrm{mL}(6-23.4), \mathrm{p}=0.004]$ and urine NGAL/creatinine ratio [39.3 (21.1-126.3) vs. 11.8 (6.3-40.9), $\mathrm{p}=0.03$ ] in diabetics with high-normal albuminuria $(\mathrm{n}=6)$ compared to those of patients with low-normal albuminuria $(\mathrm{n}=40)$.

Conclusions: Urine NGAL levels indicate early kidney injury in normoalbuminuric and normotensive adolescents with type 1 diabetes. The importance of elevated NGAL levels needs to be evaluated for future development of diabetic complications.

FC11-145 Diabetes Complications

Abstract withdrawn. 


\section{FC11-146 Diabetes Complications}

Gastric emptying disorder in children and adolescents with diabetes mellitus type 1

Elena Hennessy'; Yuriy G. Leytes ${ }^{2}$; Tamara L. Kuraeva'; Valentina Peterkova

${ }^{1}$ Endocrinology Research Centre, Institute of Paediatric Endocrinology, Moscow, Russian Federation; ${ }^{2}$ Endocrinology Research Centre, Endoscopy Department, Moscow, Russian Federation

Background: Gastric emptying disorder is a sign of gastrointestinal autonomic neuropathy in patients with DM1. A non-radioactive diagnostic method to estimate gastric emptying is $13 \mathrm{C}$ octanoic acid breath test.

Objective and hypotheses: The evaluation of motor-evacuation function of GIT as a marker of gastrointestinal autonomic neuropathy in children and adolescents with DM1.

Methods: Diagnostic 13C octanoic acid breath test was used to examine the gastric emptying rate in 45 cases with DM1: mean age $15,15 \pm 4,42$ years, mean duration of DM1 9,2 $\pm 4,4$ years, mean HbA1c 10,4 $\pm 2,2 \%$.

Results: The mean gastric half emptying time (t1/2) in total group $(n=45)$ was $87,4 \pm 70,38 \mathrm{~min}$ (normal< $75 \mathrm{~min}$ ). Delayed gastric empting (mean $\mathrm{t} 1 / 2$ $137,58 \pm 83,92 \mathrm{~min}$ ) was found in $42,2 \%$ (group 1-19 cases). 57,8\% (group $2-26$ cases) had normal gastric emptying (mean t1/2 50,8 $\pm 18,5$ min). No significant difference was found between patients of both groups in the age (mean 1 group 15,73 $\pm 3,27$ years, 2 group 14,72 $\pm 5,1$ years), duration of DM1

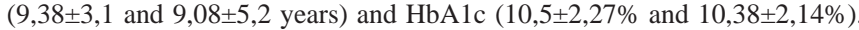
However the correlation was found in total group between $t 1 / 2$ and age $(\mathrm{R}=0,34, \mathrm{p}<0,05)$ and in group 1 between $\mathrm{t} 1 / 2$ and $\mathrm{HbA} 1 \mathrm{c}(\mathrm{R}=0,48, \mathrm{p}<0,05)$ Symptoms such as epigastric burning, nausea, heavy stomach, constipation, bloating, diarrhea are common for motor-evacuation disorders of GIT and was found in 6 cases (31,5\%) in group 1, in 4 cases (15,4\%) in group 2 $(\mathrm{R}=0,18, \mathrm{p}>0,05)$. There was a correlation between $\mathrm{t} 1 / 2$ and presence of microalbuminuria in total group $(\mathrm{R}=0,37 \mathrm{p}<0,05)$, correlation between delayed gastric emptying and presence of microalbuminuria $(>30 \mathrm{mg} / \mathrm{l})(\mathrm{R}=0,34$ $\mathrm{p}<0,05)$. Comparing two groups we found that microalbuminuria $>30 \mathrm{mg} / \mathrm{l}$ is more common for group 1 ( $g=0,02)$.

Conclusions: Our data show the existence of gastrointestinal autonomic neuropathy in children and adolescents with DM1. We found significant correlation between gastric emptying and age, gastric emptying and presence of microalbuminuria. Delayed gastric emptying correlates with $\mathrm{HbA} 1 \mathrm{c}$ and microalbuminuria.

\section{FC12-147 Growth/Acid Labile Subunit}

\section{Humanin prevents growth failure in glucocorticoid-treated mice}

Farasat Zaman' ${ }^{1}$; Chrysis Dionisios²; Bengt Fadeel'; Lars Sävendahl'

'Karolinska Institutet, Women's and Children's Health, Pediatric

Endocrinology, Astrid Lindgren Children's Hospital, Stockholm,

Sweden; 'University of Patras, Department of Pediatrics, Division of

Endocrinology, Medical School, University of Patras, Greece, Patras,

Greece; ${ }^{3}$ Karolinska Institutet, Institute of Environmental Medicine,

Division of Molecular Toxicology, Stockholm, Sweden

Background: Glucocorticoids (GCs) are used to treat multiple disorders including cancers and many inflammatory diseases. Unfortunately, GCs also exert a multitude of side-effects including bone growth impairment, an effect linked to increased apoptosis and decreased proliferation in growth plate chondrocytes. So far, no treatment preventing GC-induced growth failure has been identified.

Objective and hypotheses: We here hypothesized that humanin, known to prevent apoptosis in neurons, may protect from GC-induced chondrocyte apoptosis and bone growth impairment.

Methods: Mice (FVB and C57BL) were subcutaneously injected with dexamethasone (Dexa; $2 \mathrm{mg} / \mathrm{kg} /$ day) alone or in combination with an intraperitoneal injection of a humanin $[\mathrm{Gly}(14)]$ derivative $(\mathrm{HNG} ; 1 \mu \mathrm{g} / \mathrm{mice} /$ day $)$ for four weeks while femur growth was monitored by X-ray. For mechanistic studies, cultures of ATDC-5 chondrocytes, fetal rat metatarsal bones, human growth plate cartilage and human monocyte-derived macrophages (HMDMs) were used.

Results: Dexa significantly impaired bone growth in treated mice, an effect which was completely prevented when HNG was co-administered with Dexa $(\mathrm{P}<0.001$ vs. Dexa alone; $\mathrm{n}=10)$. As expected, Dexa increased apoptosis in growth plate chondrocytes. The co-treatment with $\mathrm{HNG}$ efficiently rescued growth plate chondrocytes from $\mathrm{GC}$-induced apoptosis $(\mathrm{P}<0.01$ vs. Dexa alone; $n=10)$. The anti-apoptotic effect of HNG was confirmed in cultured human growth plate biopsies exposed to Dexa and the bone growth rescuing effect was validated in cultured fetal rat metatarsal bones demonstrating a local HNG action in the growth plate. Experiments in lipopolysacharide-exposed HMDMs showed that HNG does not interfere with the desired anti-inflammatory effects of Dexa. Applying immunohistochemistry, we found that the humanin protein is expressed in human growth plate cartilage indicating a potential normal physiological role.

Conclusions: Applying several experimental models, we have identified a novel strategy based on the co-treatment with HNG to prevent growth failure caused by GCs.

\section{FC12-148 Growth/Acid Labile Subunit}

\section{Genetic and environmental influences on age of the Infancy-Childhood Transition (ICT)}

Alina German ${ }^{1}$; Jonatan Dubnov²; Sergey Ermakov ${ }^{3}$; Peter Inga ${ }^{4}$;

Hana Akons'; 'Livshits Gregory ${ }^{3}$; Ze'ev Hochberg ${ }^{5}$

${ }^{1}$ Clalit Health Service, Pediatric Endocrinology, Haifa, Israel; ${ }^{2}$ Ministry

of Health, District Health Office, Haifa, Israel; ${ }^{3}$ Tel Aviv University,

Sackler Faculty of Medicine, Tel Aviv, Israel; ${ }^{4}$ Mount Sinai School of

Medicine, Genetics and Genomic Sciences, New York, United States;

${ }^{5}$ Technion, Faculty of Medicine, Haifa, Israel

Background: The transition from infancy to childhood (ICT) at age 7-13 months is marked by a growth spurt, when the GH-IGF-1 axis activity sets in. Delayed ICT (DICT) explains $50 \%$ of idiopathic short stature.

Objective and hypotheses: ICT is the mechanism for predictive adaptation to energy resources; it is transmitted trans-generations and responds to weight gain in infancy.

Methods: The study examined growth pattern in 290 boys (M) and 346 girls (F), including 62 pairs of monozygotic (MZ), 112 pair dizygotic twins (DZ) and 144 pair siblings (SB). Curve fitting was used to examine continuous growth from a set of discrete measurements of length (L) and weight (W) and calculated ponderal index (PI). A model-based variance component analysis (VCA) fitted the most parsimonious model of the twins/sibs ICT determinant for genetic, common environment effects shared by sibs or shared by twins only.

Results: M ICT (at $10.5 \pm 1.8 \mathrm{mo}$ ) occurred at L $72.5 \pm 3.2 \mathrm{~cm}, \mathrm{~W} 9.1 \pm 1.0 \mathrm{~kg}$ and PI of $23.7 \pm 2.1 \mathrm{~kg} / \mathrm{m}^{3}$ after $\mathrm{W}$ gain of $6.2 \pm 0.8 \mathrm{~kg}$ and $\mathrm{L}$ gain of $24.2 \pm 3.1 \mathrm{~cm}$, as compared to F ICT (at $9.7 \pm 2.0 \mathrm{mo}, \mathrm{p}<0.0001$ ) at $\mathrm{L} 70.2 \pm 3.8 \mathrm{~cm}, \mathrm{p}<0.0001$, $\mathrm{W} 8.3 \pm 1.2 \mathrm{~kg}$ after a $\mathrm{W}$ gain of $5.6 \pm 1.0 \mathrm{~kg}, \mathrm{p}<0.001$, and $\mathrm{L}$ gain of $22.6 \pm 3.5$ $\mathrm{cm}, \mathrm{p}<0.001$. The PI $(23.9 \pm 2.4 \mathrm{~kg} / \mathrm{m} 3)$ was similar among boys and girls $(\mathrm{p}=0.45)$. After adjustments for gestational age and sex, ICT as a function of $\mathrm{W}$ gain $(\mathrm{r}=0.53, \mathrm{p}<0.01)$, L gain $(\mathrm{r}=0.68, \mathrm{p}<0.01)$ and $\mathrm{PI}(\mathrm{r}=-0.40, \mathrm{p}<0.01)$, explained $49 \%$ of ICT age. The VCA model showed no genetic influence for ICT, but strong effect for common sib environment - parents and household (37.8\%) and common twin environment - pregnancy (4.7\%).

Conclusions: As a major determinant of adult height, the ICT is not genetically influenced. It depends mostly on non-genetic parental/environmental factors shared by siblings, infantile weight gain and a critical PI. 


\section{FC12-149 Growth/Acid Labile Subunit \\ IGFALS mutations represent the most frequent molecular defect in a cohort of patients with primary IGF-I deficiency and postnatal growth deficit}

Silvia de Frutos'; Eva Barroso²; Ana Gómez';

Ricardo Gracia Bouthelier'; Elena Gallego-Gómez'; Karen E. Heath ${ }^{2}$ $\underline{\text { Ángel Campos-Barros }}^{2}$

1 University Hospital La Paz, Institute of Medical and Molecular Genetics, INGEMM, Madrid, Spain; ${ }^{2}$ University Hospital La Paz, IdiPAZ and CIBER de Enfermedades Raras, Unidad 753, Instituto de Salud Carlos III, Institute of Medical and Molecular Genetics, INGEMM, Madrid, Spain; ${ }^{3}$ University Hospital La Paz, Pediatric Endocrinology, Madrid, Spain; ${ }^{4}$ Hospital University 12 de Octubre, Pediatric Endocrinology, Madrid, Spain

Background: Approximately $25 \%$ of proportionate short stature patients present with IGF-I deficiency in the face of normal or supranormal GH response tests, a condition defined as primary or idiopathic IGF-I deficiency. Dysfunctional GH1 variants, mutations in the GHR coding gene, GHR, or in $S T A T 5 b$, implicated in the post receptor signalling pathway, explain a very small fraction of cases with primary IGF-I deficiency. On the other hand, recent reports have shown that mutations in IGFALS (16p13.3), encoding ALS, may play a role in the aetiology of primary IGF-I deficiency and short stature, indicating that ALS plays an important role regulating IGF-I bioavailability during postnatal growth.

Objective and hypotheses: To investigate the frequency of IGFALS mutations in a cohort of children with primary IGF-deficiency and short stature without a known molecular defect.

Methods: Subjects: 80 children with postnatal growth deficit (<-2.0 SDS); decreased IGF-I ( $<-1.5$ SDS) and normal or supranormal GH response tests. Molecular studies: Mutation screening of coding sequences, intron/exon boundaries and known regulatory regions of IGFALS, GHR, IGFBP3 and IGF1 by HRM and DNA sequencing.

Results: We identified a total of 9 families with IGFALS mutations (see table), presenting 7 different mutations, 4 of which previously undescribed (p.S274F, p.R63G, p.L15dup and p.R493H). Four unrelated families presented with the same mutation, N276S, previously reported.

\begin{tabular}{lcccc} 
GENE & $\begin{array}{c}\text { NR. OF MUTA- } \\
\text { TIONS }\end{array}$ & $\begin{array}{c}\text { NR. HOMOZY- } \\
\text { GOUS }\end{array}$ & $\begin{array}{c}\text { NR. COMPOUND } \\
\text { HETEROZYGOUS }\end{array}$ & $\begin{array}{c}\text { NR. HE- } \\
\text { TEROZY- } \\
\text { GOUS }\end{array}$ \\
\hline IGFALS & $\mathbf{9} / 80(11.25 \%)$ & 4 & 1 & 4 \\
GHR & $\mathbf{2} / 80(2.5 \%)$ & 0 & 0 & 2 \\
IGFBP3 & $\mathbf{1 / 8 0}(1.25 \%)$ & 0 & 0 & 1 \\
IGF1 & $\mathbf{1} / 80$ & 0 & 0 & 0 \\
\hline
\end{tabular}

Conclusions: IGFALS mutations represent the most frequent molecular defect $(11.25 \%)$ in the studied cohort of primary IGF-I deficient patients with short stature. The detection of heterozygous IGFALS mutations in patients with decreased IGF-I, IGFBP-3 and ALS levels and postnatal growth deficit supports the hypothesis that ALS haploinsufficiency may be a factor involved in the aetiology of moderate postnatal growth deficit associated with partial IGF-I deficiency.

\section{FC12-150 Growth/Acid Labile Subunit}

Heterozygous non-synonymous IGFALS allelic variants associated with diminished levels of all three IGF-I, IGFBP-3 and ALS are found in non-familial (NF) but not in familial (F) idiopathic short stature (ISS) children

Alicia Martínez'; Paula Scaglia'; Ana Keselman²;

María Gabriela Ropelato2; María Gabriela Ballerini; Sonia Bengolea ${ }^{3}$; Viviana Pipman ${ }^{4}$; Rodolfo Rey ${ }^{1}$; Juan Heinrich²; Héctor Jasper'; Horacio Domené

${ }^{1}$ CONICET, CEDIE, Hospital de Niños Ricardo Gutiérrez, Buenos Aires, Argentina; ${ }^{2}$ Hospital de Niños Ricardo Gutiérrez, División Endocrinología, Buenos Aires, Argentina; ${ }^{3} \mathrm{H}$ ospital J. Fernández, Servicio de Pediatría, Buenos Aires, Argentina; ${ }^{4}$ Hospital E. Tornú, Servicio de Pediatría, Buenos Aires, Argentina

Background: Idiopathic short stature (ISS) is essentially a diagnosis of exclusion, probably including several as yet unrecognized conditions. Previous studies have shown that heterozygous carriers (HC) for non-synonymous IGFALS allelic variants (ns-AV) are frequent in ISS children.

Objective and hypotheses: In this study we compared auxological and biochemical data in ISS children classified as familial (F) and non-familial (NF) and according to the level of IGF-I aiming to: 1) determine differences in height (H-SDS), target adjusted height (TAH-SDS), height gain during follow-up $(\Delta \mathrm{H}-\mathrm{SDS}=$ Last prepubertal H-SDS - H-SDS at admission $)$ and levels of components of the IGF system; 2) look for the most likely candidates for carriers of IGFALS allelic variants.

Methods: Auxological data were collected in 68 ISS prepubertal children. Serum levels of IGF-I (RIA), IGFBP-3 (IRMA), and ALS (RIA) were determined and the IGFALS gene was sequenced. ISS children were classified as $\mathrm{F}$ and NF, using -1.6 as TAH-SDS cut-off, or alternatively as having either low or normal IGF-I levels (<-2.0 SDS). Statistical analysis: paired or unpaired T test, Mann Whitney and Chi square test were used.

Results: At admission, NF were significantly shorter than $\mathrm{F}$ children, but levels of IGF-I, IGFBP-3 and ALS were not significantly different. When classified according to IGF-I level, those with low IGF-I had reduced levels of IGFBP-3 and ALS but no differences in H-SDS or TAH-SDS. Data are expressed as mean $\pm \mathrm{SD}$.

\begin{tabular}{|c|c|c|c|c|c|c|c|c|c|}
\hline & F-ISS & NF-ISS & $\begin{array}{c}\text { Fv vs } \\
\text { NF-ISS } \\
\text { (p) }\end{array}$ & $\begin{array}{l}\text { Normal } \\
\text { IGF-I } \\
\text { ISS }\end{array}$ & $\begin{array}{l}\text { Low } \\
\text { IGF-I } \\
\text { ISS }\end{array}$ & $\begin{array}{l}\text { Normal } \\
\text { vs low } \\
\text { IGF-I } \\
\text { ISS (p) }\end{array}$ & $\begin{array}{c}\text { ISS } \\
\text { with } \\
\text { ns-AV }\end{array}$ & $\begin{array}{c}\text { ISS } \\
\text { without } \\
\text { ns-AV }\end{array}$ & $\begin{array}{l}\text { With } \\
\text { vs wi- } \\
\text { thout } \\
\text { ns-AV } \\
\text { (p) }\end{array}$ \\
\hline $\mathrm{N}$ & 34 & 34 & & 44 & 24 & & 8 & 60 & \\
\hline $\mathrm{CA}(\mathrm{y})$ & $\begin{array}{c}8,18 \\
\pm 2,57\end{array}$ & $\begin{array}{c}8,57 \\
\pm 3,24\end{array}$ & NS & $\begin{array}{c}8,66 \\
\pm 3,12\end{array}$ & $\begin{array}{c}7,85 \\
\pm 2,45\end{array}$ & NS & $\begin{array}{c}8,04 \\
\pm 2,11\end{array}$ & $\begin{array}{c}8,42 \\
\pm 3,01\end{array}$ & NS \\
\hline H-SDS & $\begin{array}{l}-2,65 \\
\pm 0,45\end{array}$ & $\begin{array}{l}-2,96 \\
\pm 0,49\end{array}$ & 0.0080 & $\begin{array}{l}-2,77 \\
\pm 0,57\end{array}$ & $\begin{array}{l}-2,86 \\
\pm 0,31\end{array}$ & NS & $\begin{array}{l}-2,94 \\
\pm 0,31\end{array}$ & $\begin{array}{l}-2,78 \\
\pm 0,51\end{array}$ & NS \\
\hline TAH-SDS & $\begin{array}{l}-0,97 \\
\pm 0,47\end{array}$ & $\begin{array}{l}-2,38 \\
\pm 0,58\end{array}$ & - & $\begin{array}{l}-1,61 \\
\pm 0,87\end{array}$ & $\begin{array}{l}-1,79 \\
\pm 0,91\end{array}$ & NS & $\begin{array}{l}-2,17 \\
\pm 0,74\end{array}$ & $\begin{array}{l}-1,61 \\
\pm 0,89\end{array}$ & NS \\
\hline $\begin{array}{l}\text { IGF-I } \\
\text { (SDS) }\end{array}$ & $\begin{array}{l}-1,20 \\
\pm 1,33\end{array}$ & $\begin{array}{l}-1,84 \\
\pm 1,45\end{array}$ & NS & $\begin{array}{l}-0,67 \\
\pm 0,82\end{array}$ & $\begin{array}{l}-3,07 \\
\pm 0,85\end{array}$ & - & $\begin{array}{l}-2,16 \\
\pm 1,93\end{array}$ & $\begin{array}{l}-1,43 \\
\pm 1,33\end{array}$ & NS \\
\hline $\begin{array}{l}\text { IGFBP-3 } \\
\text { (SDS) }\end{array}$ & $\begin{array}{l}-0,93 \\
\pm 0,92\end{array}$ & $\begin{array}{l}-1,05 \\
\pm 1,45\end{array}$ & NS & $\begin{array}{l}-0,67 \\
\pm 0,99\end{array}$ & $\begin{array}{l}-1,36 \\
\pm 1,59\end{array}$ & 0.0313 & $\begin{array}{l}-2,09 \\
\pm 1,58\end{array}$ & $\begin{array}{l}-0,85 \\
\pm 1,08\end{array}$ & 0.0052 \\
\hline $\begin{array}{l}\text { ALS } \\
\text { (SDS) }\end{array}$ & $\begin{array}{l}-1,23 \\
\pm 1,17\end{array}$ & $\begin{array}{l}-1,62 \\
\pm 1,58\end{array}$ & NS & $\begin{array}{l}-1,00 \\
\pm 1,08\end{array}$ & $\begin{array}{c}-2,2 \\
\pm 1,59\end{array}$ & 0.0005 & $\begin{array}{l}-2,78 \\
\pm 1,77\end{array}$ & $\begin{array}{l}-1,24 \\
\pm 1,25\end{array}$ & 0.0028 \\
\hline ns-AV & $2 / 34$ & $6 / 34$ & NS & $3 / 44$ & $5 / 24$ & NS & & & \\
\hline Follow up & N 21 & N 21 & $\begin{array}{c}\text { T test } \\
(p)\end{array}$ & N 25 & N 17 & $\begin{array}{c}\text { T test } \\
(p)\end{array}$ & & & \\
\hline$\triangle \mathrm{H}-\mathrm{SDS}$ & $\begin{array}{c}0,19 \\
\pm 0,31\end{array}$ & $\begin{array}{c}0,49 \\
\pm 0,34\end{array}$ & 0.0046 & $\begin{array}{c}0,39 \\
\pm 0,39\end{array}$ & $\begin{array}{c}0,25 \\
\pm 0,28\end{array}$ & NS & & & \\
\hline $\begin{array}{l}\Delta \text { Time (y, } \\
\text { length of } \\
\text { prepuber- } \\
\text { tal follow } \\
\text { up period) }\end{array}$ & $\begin{array}{c}2,06 \\
\pm 1,05\end{array}$ & $\begin{array}{c}2,68 \\
\pm 1,20\end{array}$ & NS & $\begin{array}{c}2,49 \\
\pm 1,23\end{array}$ & $\begin{array}{r}2,18 \\
\pm 1,06\end{array}$ & NS & & & \\
\hline
\end{tabular}

Follow up could be evaluated in 42/68 ISS children (21 F, 21 NF). NF children were shorter than $\mathrm{F}$ at admission and showed a higher growth gain during follow up. IGFALS ns-AV were present in 6/34 NF vs 2/34 F (p NS), but if low IGF-I and IGFBP-3 were also considered, IGFALS ns-AV were present in $5 / 34 \mathrm{NF}$ and $0 / 34 \mathrm{~F}(\mathrm{p}=0.02)$. Same results were obtained if low ALS was added.

Conclusions: These findings suggest that the NF subgroup of ISS children with reduced levels of IGF-I and IGFBP-3, would be the most likely candidate for genotyping the IGFALS gene searching for ns-AV. 


\section{FC12-151 Growth/Acid Labile Subunit}

Serum levels of acid-labile subunit and growth in short children born small for gestational age

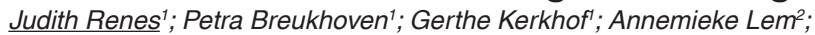
Jaap van Doorn ; Anita Hokken-Koelega ${ }^{2}$

'Erasmus MC - Sophia Children's Hospital, Paediatric Endocrinology, Rotterdam, Netherlands; ${ }^{2} E$ rasmus MC - Sophia Children's Hospital

/ Dutch Growth Research Foundation, Paediatric Endocrinology, Rotterdam, Netherlands; ${ }^{3}$ University MC Utrecht, Metabolic and

Endocrine Diseases, Utrecht, Netherlands

Background: Acid Labile Subunit (ALS) forms a ternary complex with IGFI and IGFBP-3. In the absence of ALS, IGF-I and IGFBP-3 levels are reduced. Short children born small for gestational age (SGA) have lower IGF-I and IGFBP-3 levels than healthy peers. Growth hormone treatment (GH) in these children results in normalisation of IGF-I and IGFBP-3 levels and in significant catch-up growth. Responsiveness to $\mathrm{GH}$ shows a wide variation, the contribution of ALS is unknown.

Objective and hypotheses: To analyze serum ALS levels and their association with 1-year growth in short SGA treated with GH.

Methods: IGFBP-3 levels were measured at start of GH in 347 short SGA children (mean age $8.0 \mathrm{yr}$ ). We determined ALS levels in children with an IGFBP-3 $\leq-1$ SDS. Anthropometry was performed every three months. Subjects were treated with GH 1 or $2 \mathrm{mg} / \mathrm{m} 2 /$ day. To study the relation between baseline ALS levels and height gain SDS, multiple linear regression analyses were performed.

Results: At baseline, IGFBP-3 levels were $\leq-1$ SDS in $178(52 \%)$ short SGA children. Mean (SD) ALS level in these subjects was - 1.7 (0.9) SDS; 52 children $(30 \%)$ had an ALS level $\leq-2$ SDS (low ALS). Subjects with low ALS had a significantly lower gestational age $(\mathrm{P}=0.02)$ and were shorter at birth $(\mathrm{P}=0.04)$ than subjects with ALS $-2>$ SDS $\leq 2$. They were also significantly younger $(\mathrm{P}<0.001)$ and had lower height SDS $(\mathrm{P}=0.04)$ and BMI SDS $(\mathrm{P}$ $=0.02$ ) at start of GH. After adjustment for confounders (Table 1), 1-year height gain SDS during GH was positively associated with ALS SDS at start $(\beta=0.367, P=0.002)$.

Conclusions: Serum ALS levels $\leq-2$ SDS are often found in short SGA children. After adjustment, ALS levels are positively associated with 1-year height gain in short SGA children treated with GH.

\begin{tabular}{lcc}
$\begin{array}{l}\text { Table } \mathbf{1} \text { Multiple regression model for gain in } \\
\text { height SDS during } \mathbf{1} \text { year of } \mathrm{GH}(\mathbf{n = 1 3 9 )}\end{array}$ & & \\
\hline Gender & Beta & P-value \\
\hline Age & -0.040 & 0.378 \\
\hline Height SDS & -0.079 & $<\mathbf{0 . 0 0 1}$ \\
\hline BMI SDS & -0.054 & 0.144 \\
\hline TH SDS & -0.016 & 0.366 \\
\hline IGF-I SDS & 0.026 & 0.256 \\
\hline IGFBP-3 SDS & 0.050 & 0.253 \\
\hline Mean GH dose (mg/m2/day) & 0.138 & 0.254 \\
\hline ALS SDS & -0.164 & 0.647 \\
\hline Age samples & 0.367 & $\mathbf{0 . 0 0 2}$ \\
\hline R square & -0.022 & 0.274 \\
\hline R square adjusted & & \\
\hline
\end{tabular}

Variables are at start of GH, except for mean GH dose. Children who reached puberty during follow-up or had no available parental height were excluded from analysis. The model included the interaction terms: ALS SDS $\times$ IGF-I SDS; ALS $\times$ age samples; mean GH dose $\times$ IGFBP-3 SDS.

\section{FC12-152 Growth/Acid Labile Subunit}

\section{Role of the IGFALS gene in pubertal onset}

Ora Hess' ${ }^{1}$ : Morad Khayat'; Stavit Allon-Shalev²; Yardena Tenenbaum-Rakover ${ }^{1}$

${ }^{1}$ Ha'Emek Medical Center, Pediatric Endocrine Unit, Afula, Israel;

'Ha'Emek Medical Center, Genetic Institute, Afula, Israel

Background: The protein acid-labile subunit (ALS) binds to IGF-I, its main role being extension of the half life of circulating IGF-I. Mutation of the IGFbinding ALS gene (IGFALS) results in short stature phenotype. The other functions of ALS are not yet clear.
Objective and hypotheses: To date, only 16 mutations of the IGFALS gene have been reported. In the current study, we describe two siblings of consanguineous union with a novel homozygous mutation of the IGFALS gene. Methods: Detailed phenotypic description, biochemical parameters and IGFALS gene sequencing are reported.

Results: The proband, at 16 years of age, was -2.9 SDS in height and -4.5 SDS in weight with bone age delayed by 2 years. Exaggerated stimulated GH (38 ng/ml), low IGF-I and IGFBP-3 $(<25 \mathrm{ng} / \mathrm{ml}$ and $<500 \mathrm{ng} / \mathrm{ml}$, respectively) and no response to GH injections in IGF-I generation test indicated GH insensitivity. Testicular volume of $2 \mathrm{ml}$ and blunted gonadotropin response to GnRH pointed to hypogonadism. His 13-year-old sister was -2.8 SDS in height and -3.75 SDS in weight without pubertal signs. She had low IGF-I and IGFBP-3 (44.5 ng/ml and $<500 \mathrm{ng} / \mathrm{ml}$, respectively), exaggerated peak GH $(29 \mathrm{ng} / \mathrm{ml})$ in response to stimulation test and blunted gonadotropin response to GnRH. The proband and his sister had markedly low serum ALS (3.9, 4.1 $\mathrm{mU} / \mathrm{ml}$, respectively). Sequencing of the IGFALS gene revealed a novel homozygous mutation in both siblings, which was found in heterozygous form in the mother. The c.380T $>\mathrm{C}$ mutation in exon 2 resulted in substitution of leucine with proline at position 127 . The findings of delayed puberty in both siblings points to a main role for ALS in pubertal onset, most likely mediated by IGF-I.

Conclusions: Our distinct findings emphasize the main role of ALS and IGFI in pubertal onset in addition to its known function in the growth process.

\section{FC13-153 Puberty \\ Neonatal exposure to diethylstilbestrol decreases gonadotropin-releasing hormone sensitivity to leptin in immature female rats Anne-Simone Parent; Elise Naveau; Marie-Christine Lebrethon; Arlette Gérard; Jean-Pierre Bourguignon \\ University of Liège, GIGA-Neurosciences, Liège, Belgium}

Background: Neuroendocrine disruption has emerged as a component of endocrine disrupting chemical (EDC) effects on sexual maturation, reproduction and energy balance. The hypothalamus is a single place for early programming of reproduction and energy balance and leptin has appeared to be a possible organizer in the control of energy balance during fetal and neonatal life. Objective and hypothesis: We hypothesized that neonatal exposure to diethylstilbestrol (DES), an estrogenic EDC that causes alteration of sexual maturation and obesity later in life could reduce hypothalamic sensitivity to leptin. Methods: Newborn female rats were exposed to vehicle (corn oil) or DES (1mg/kg, subcutaneous injection) from postnatal day (PND) 1 to 5 . Hypothalamic explants from control and exposed animals were dissected at PND 15 and incubated individually for 4 hours. The medium was collected every 7.5 min and GnRH concentration was measured by radioimmunoassay.

Results: We confirm our previous findings that leptin increases pulse frequency of GnRH secretion in immature female rats (PND 15). Those data underscore once more the involvement of energy balance-related peptides in the regulation of reproduction.

We also show here that early exposure to DES does not affect pulsatile GnRH secretion in immature female rats. At PND 15, neither GnRH interpulse interval nor GnRH response to glutamate was affected.

However, the facilitating effect of leptin on GnRH secretion $(44 \pm 3$ vs $60 \pm 0$ min interpulse interval, leptin vs control) in vitro at PN15 was decreased after neonatal exposure to DES ( $51 \pm 3$ vs $61 \pm 2.5$ min interpulse interval, leptin vs control).

Conclusion: Neonatal exposure to DES induces a decrease of GnRH secretion sensitivity to the stimulatory effect of leptin.

We are now studying whether prenatal food restriction could sensitize the newborn to the neuroendocrine effect of DES on hypothalamic sensitivity to regulating factors such as leptin. 


\section{FC13-154 Puberty \\ Exposure to endocrine disrupting chemicals affects the pubertal regulatory network \\ Johanna Mueller'; Sabine Heger ${ }^{2}$ \\ ${ }^{1}$ Hannover Medical School, Institute of Clinical Biochemistry, Hanover, Germany; ${ }^{2}$ Children's Hospital "Auf der Bult", Pediatric Endocrinology and Diabetology, Hanover, Germany}

Background: Endocrine disrupting chemicals (EDCs) are exogenous substances interfering with the endocrine system imitating or antagonizing the action of endogenous hormones. Today it is clear that environmental toxins affect embryonic development and subsequent gonadal maturation, fertility, and the timing of puberty not only of the first but additionally of subsequent generations in humans and rodents.

Objective and hypotheses: In our study we investigate the effect of EDCs on genes involved in the pubertal development. We analyzed ß-Estradiol (E2), that is present in water and food, the plasticizer Bisphenol A (BPA), and the phytoestrogen Genistein. These three compounds have been reported to advance the pubertal process. Furthermore, we studied the effect of dioxin that conducts its effects via the aryl hydrocarbon receptor and xenobiotic response elements (XRE) resulting in delayed puberty.

Methods: We utilized Dual Luciferase assays to investigate the direct effect of single EDCs and a mixture of EDCs on KiSS1 and EAP1 expression, both part of the gonadotropin releasing hormone neuronal network.

Results: Treatment with BPA $(10 \mu \mathrm{M}, \mathrm{n}=4)$ stimulated both the KiSS1 promoter (556bp) and EAP1 promoter (2744bp) construct via the classical estrogenic signaling pathway utilizing $\mathrm{ER} \alpha$.

Furthermore, a stimulatory effect was observed through alternative estrogenic mechanisms after treatment with ß-Estradiol (E2; 100nM, n=4), Genistein (20 $\mu \mathrm{M}, \mathrm{n}=4)$ and an EDC-Mix. In contrast, treatment of the KiSS1 promoter with $5 \mathrm{nM}$ dioxin $(\mathrm{n}=4)$ inhibited KiSS1 expression specifically via a XRE binding site located 464bp upstream of the transcription start site. No dioxin effect was observed on EAP1 expression due to lack of a XRE binding site in the EAP1 promoter region.

Conclusions: Taken together, our study provides new insights in molecular mechanisms employed by environmental factors interfering with the pubertal regulatory network.

\section{FC13-155 Puberty \\ Significant increase in serum estrogenic bioactivity in 9 infant girls with isolated premature thelarche: a consequence of environmental pollutant exposure? \\ Francoise Paris; Laura Gaspari; Charles Sultan \\ CHU Montpellier et Université Montpellier 1, Unité d'Endocrinologie- \\ Gynécologie Pédiatrique, Hôpital Arnaud-de-Villeneuve; Service \\ d'Hormonologie, Hôpital Lapeyronie, Montpellier, France}

Background: Isolated premature thelarche (IPT) is usually a benign condition that disappears without influencing target height or the timing of puberty. The pathophysiology of IPT remains elusive, although recent investigation indicated the role of environmental pollutants in female premature breast development, since most of these pollutants present estrogenic activity. We recently investigated 9 infant girls with IPT whose parents reported occupational or environmental exposure to endocrine-disruptor chemicals (EDCs) before/during pregnancy and after the patients' birth.

Objective and hypotheses: The aim of the present study was to further analyze the pathophysiology of IPT by assessing the impact of pre/postnatal exposure to EDCs in 9 patients through evaluation of serum estrogenic bioactivity.

Methods: 1) Plasma estradiol (E2), follicle-stimulating hormone (FSH), and luteinizing hormone ( $\mathrm{LH})$ were measured at baseline and after GnRH testing; bone age was assessed and pelvic ultrasonography was performed. 2) Estrogenic bioactivity of patient serum was analyzed with an ultrasensitive bioassay (JCEM, 2002).

Results: 1) Clinical, endocrine and imaging findings of the 9 infant girls with IPT are presented in Table 1.2) The mean estrogenic bioactivity was significantly elevated $(12.31 \pm 7.05 \mathrm{pg} / \mathrm{mL})$ vs. controls matched for age $(3.53 \pm 2.23$ $\mathrm{pg} / \mathrm{mL}$; JCEM,2002) $(p<0.01)$.

Conclusions: The significant increase in serum estrogenic bioactivity in these 9 infant girls with IPT strongly suggests that premature female breast de- velopment may be related in some cases to pre/postnatal contamination by environmental xenoestrogens.

\begin{tabular}{|c|c|c|c|c|c|c|c|c|c|c|c|}
\hline Patients & $\begin{array}{c}\text { Age } \\
\text { (years/ } \\
\text { months) }\end{array}$ & $\begin{array}{l}\text { Tanner } \\
\text { stage }\end{array}$ & $\begin{array}{l}\text { Growth } \\
\text { rate }\end{array}$ & $\begin{array}{l}\text { Bone } \\
\text { age }\end{array}$ & $\begin{array}{l}\text { Uterus } \\
\text { length } \\
\text { at pelvic } \\
\text { US } \\
(\mathrm{mm})\end{array}$ & $\begin{array}{l}\mathrm{E} 2 \\
\text { (pg/ } \\
\mathrm{mL})\end{array}$ & $\begin{array}{l}\text { Basal } \\
\text { FSH } \\
(\mathrm{mlU} / \\
\mathrm{mL})\end{array}$ & $\begin{array}{c}\text { Basal } \\
\text { LH } \\
(\mathrm{mlU} / \\
\mathrm{mL})\end{array}$ & $\begin{array}{c}\text { Peak } \\
\text { FSH/LH } \\
\text { at GnRH } \\
\text { test } \\
\text { (mlU/mL) }\end{array}$ & $\begin{array}{l}\text { Parents' } \\
\text { occupational } \\
\text { or envi- } \\
\text { ronmental } \\
\text { exposure to } \\
\text { EDCs }\end{array}$ & $\begin{array}{c}\text { Estrogenic } \\
\text { bioactivity } \\
\text { (pg/mL) }\end{array}$ \\
\hline 1 & $1 \mathrm{yr} / 8 \mathrm{mo}$ & B3P1 & $\mathrm{N}$ & $\mathrm{N}$ & 18 & $<9$ & 5.5 & $<0.5$ & $44.1 / 5.3$ & $\begin{array}{l}\text { commercial } \\
\text { painter }\end{array}$ & 16.3 \\
\hline 2 & $1 \mathrm{yr} / 4 \mathrm{mo}$ & B3P1 & $\uparrow$ & $\uparrow$ & 35 & $<9$ & 5.6 & $<0.5$ & $28.1 / 7.5$ & countryside & 17.7 \\
\hline 3 & $7 \mathrm{yr} / 2 \mathrm{mo}$ & B2-3P1 & $\mathrm{N}$ & $\uparrow$ & 37 & $<9$ & 4.3 & 1.2 & $11.3 / 7.9$ & farmer & 3.5 \\
\hline 4 & $3 \mathrm{yr} / 2 \mathrm{mo}$ & B2P1 & $\mathrm{N}$ & $\mathrm{N}$ & 28 & $<9$ & 3.7 & $<0.5$ & $35.9 / 6.3$ & $\begin{array}{l}\text { commercial } \\
\text { painter }\end{array}$ & 18.0 \\
\hline 5 & $1 \mathrm{yr} / 9 \mathrm{mo}$ & B2P1 & $\mathrm{N}$ & $\uparrow$ & 26 & $<9$ & 0.6 & $<0.5$ & ND & countryside & 5.4 \\
\hline 6 & $1 \mathrm{yr} / 5 \mathrm{mo}$ & B2-3P1 & $\uparrow$ & $\uparrow$ & 18 & 10 & 2.1 & $<0.5$ & $18.6 / 4.5$ & countryside & 7.8 \\
\hline 7 & $1 \mathrm{yr} / 6 \mathrm{mo}$ & B3P1 & $\uparrow$ & $\uparrow$ & 27 & 11 & 3.3 & $<0.5$ & 19.9/5.1 & $\begin{array}{c}\text { water } \\
\text { purification } \\
\text { station }\end{array}$ & 9.5 \\
\hline 8 & $1 \mathrm{yr} / 9 \mathrm{mo}$ & B2P1 & N & $\uparrow$ & 24 & 12 & 1.0 & $<0.5$ & ND & mechanic & 24.5 \\
\hline 9 & $1 \mathrm{yr} / 7 \mathrm{mo}$ & B2P1 & $\uparrow$ & $\uparrow$ & 30 & $<9$ & 2.6 & $<0.5$ & $33.7 / 4.1$ & countryside & 8.1 \\
\hline
\end{tabular}

\section{FC13-156 Puberty}

\section{Effect of Xbal and Pvull polymorphisms of the Estrogen Receptor a (ERa) on the onset of puberty in girls}

Olga Karapanou'; Anna Papadopoulou'; George Mastorakos²;

Andreas Fretzayas ${ }^{1}$; Poluxeni Nicolaidou'; ${ }^{1}$ Anastasios Papadimitriou ${ }^{1}$ 'University of Athens, Attikon University Hospital, Third Department of Pediatrics, Athens, Greece; '2University of Athens, Aretaieion Hospital, Endocrinology, Diabetes and Metabolism Unit, Athens, Greece

Background: Single nucleotide polymorphisms (SNPs) XbaI and PvuII of the estrogen receptor a (ERa) have been reported that they may affect age at menarche.

Objective: To examine the prevalence of these polymorphisms in girls with idiopathic precocious puberty (IPP).

Methods: We studied 41 girls with IPP, i.e. breast development $<8$ years of age, and 50 young women with pubertal maturation within normal age, who served as controls. In all IPP girls GnRH stimulation test, pelvic ultrasonography and MRI imaging of hypothalamic-pituitary area were performed. Controls were asked about their age at menarche. Both patients and controls were genotyped for XbaI and PvuII polymorphisms of the ERa gene.

Results: There was no significant difference in XbaI and PvuII polymorphisms between patients and controls. In IPP girls onset of puberty was significantly earlier for homozygous carriers of the XbaI polymorphism, x: 6.5 (1.07) years compared to hemizygous x:7.53 (0.74) years or non-carriers $\mathrm{x}$ 7.17 (0.78) years $(p=0.015)$. Pubertal onset was also earlier for homozygous carriers of both XbaI and PvuII polymorphisms, x: 5.49 (0.95) years compared to non-carriers, $x: 7.16(0.7)$ years $(p=0.002)$. Menarcheal age of controls was not significantly influenced by the presence of either XbaI $(p=0.8)$ or PvuII polymorphism ( $\mathrm{p}=0.97)$.

Conclusions: XbaI and PvuII polymorphisms, are not related to IPP in girls. However, in IPP girls the presence of homozygosity for XbaI polymorphism (xx) and to a lesser extend to PvuII polymorphism (pp) resulted in earlier onset of puberty than heterozygous or non-carriers of these polymorphisms. This effect was amplified in the girls that were homozygous for both SNPs. We speculate that these SNPs upregulate estrogen-dependent expression of kisspeptin gene and consequently facilitate kisspeptin-GRP54-GnRH mediated signaling, this effect occurring only on the ground of increased IGF-1 (common in precocious puberty), which is known to upregulate kiss 1 expression.

Horm Res 2011;76(suppl 2) 


\section{FC13-157 Puberty}

\section{Successful treatment of hypogonadotropic hypogonadism and induction of menstrual cycles in an adolescent with leptin-deficiency by substitution with recombinant methionyl \\ human leptin}

Julia von Schnurbein'; Anja Moss'; Stella A Nagel'; Hannes Mühleder²; Sadaf Farooqi ${ }^{3}$ : Martin Wabitsch'

'University Medical Center UIm, Department of Pediatrics and Adolescent Medicine, Ulm, Germany; ${ }^{2}$ Children's Hospital Wels, Department of Pediatrics and Adolescent Medicine, Wels, Austria ${ }^{3}$ University of Cambridge Metabolic Research Laboratories, Institute of Metabolic Science, Cambridge, United Kingdom

Background: Leptin-deficiency has been reported in 15 individuals worldwide. In the reported adults, midluteal phase defect / reduced testicular volume despite of normal gonadotropin levels have been seen which improved under leptin-therapy. All previously published children with leptin-deficiency were pre-pubertal with physiologically low gonadotropins prior to therapy. They experienced a timely induction of puberty through the progression of time under leptin-therapy. In 2010, we reported the first connatal leptin-deficient adolescent girl with clinical and chemical proven hypogonadotropic hypogonadism at the age of 14.8 years.

Objective and hypotheses: We here evaluate the effect of recombinant methionyl human leptin-substitution on pubertal development in a leptin naive adolescent with connatal leptin-deficiency, which has not been published before.

Methods: LH and FSH-levels were assessed before and after stimulation with gonadotropin-releasing-hormone. Nocturnal pulsatile secretion was assessed with blood samples drawn every 10 minutes.

Results: Prior to leptin-therapy, the patient had pre-pubertal basal and stimulated $\mathrm{LH}$ (basal $<0.1 \mathrm{U} / \mathrm{l}$, stimulated $0.09 \mathrm{U} / \mathrm{l}$ ) and FSH-levels (basal 0.23 U/l, stimulated 0.49) and no nocturnal pulsatile secretion of LH and FSH. After 11 weeks of substitution basal and stimulated LH and FSH-levels rose to pubertal values (basal LH $2.63 \mathrm{U} /$ l, stimulated $15.81 \mathrm{U} /$; basal FSH 3.15 $\mathrm{U} / \mathrm{l}$, stimulated $7.0 \mathrm{U} / \mathrm{l}$ ) and nocturnal pulsatility was induced. After 76 weeks of leptin-substitution, menstruation occurred at 16.3 years.

Conclusions: We describe here the first adolescent with complete hypogonadotropic hypogonadism due to connatal leptin-deficiency. The patient showed a rapid induction of gonadotropin secretion and pulsatility under leptin-substitution and a fast progression of pubertal development with induction of menarche. These data are a further prove of concept indicating that leptin is needed for a timely maturation of the hypothalamic/pituitary/gonadal axis in men as shown already convincingly in animal experiments.

\section{FC13-158 Puberty \\ Possibly damaging FGFR1 variants in Brazilian patients with Severe Congenital Hypopituitarism}

\section{Fernanda A Correa; Ericka B Trarbach; Ana Latronico;}

Luciani Carvalho; Marcela França; Aline Otto; Everlayny F Costalonga Vinicius N. Brito; Mirian Y Nishi; Ivo J. P. Arnhold;

Berenice $B$ Mendonca

Hospital das Clinicas da Faculdade de Medicina da Universidade de Sao Paulo, Laboratorio de Hormonios e Genetica Molecular LIM 42, Unidade de Endocrinologia do Desenvolvimento, Sao Paulo, Brazil

Background: FGFR1 gene is crucial for GnRH neuronal development and recent reports suggest that it could also be important for normal hypothalamic-pituitary development.

Objective and hypotheses: To investigate FGFR1 loss-of-function mutations in patients with combined pituitary hormone deficiencies (CPHD).

Methods: The entire coding region of FGFR1 was evaluated in a 107 patients (66 males) with CPHD using automatic sequencing.

Results: Two FGFR1 missense variants (p.S107L and p.R448L) were identified in heterozygous state in two unrelated female patients born from no consanguineous parents. These variants were not found in 100 healthy controls individuals. The FGFR1 p.S107L variant $($ c. $320 \mathrm{C}>\mathrm{T})$ was identified in a 43 ys old adult female with Japanese ascendancy presenting severe GH and gonadotropin deficiencies. Neuroimaging showed small anterior pituitary, normal stalk and undescended posterior pituitary. This p.S107Lmutation was previously described in patients with isolated hypogonadotropic hypogonadism (IHH). The novel p.R448L (c.1343G>T) was found in a 12 ys old Caucasian girl with GH and TSH deficiencies and Tanner I stage of puberty. MRI revealed small anterior pituitary, absent stalk and undescended posterior pituitary. In silico analysis predicted that these changes are benign using Polyphen and SIFT tools but both variants were considered damaging using Mutation Tasker tool.

Conclusions: We identified two FGFR1 variants in two unrelated females with severe congenital hypopituitarism. The role of FGFR1 a gene traditionally involved in IHH in the etiology of CPHD remains to be explained by further studies.

\section{FC14-159 Thyroid}

\section{Analysis of the thyroid phenotype in a mice model for trisomy 21. Study of the relationship between Dyrk1A overexpression and the Shh signaling pathway}

Mercè Martin ${ }^{1}$; Dulanjalee Kariyawasam ${ }^{1}$; Aurore Carré' Latif Rachdi' $^{1}$. Nathalie Janel'; Jean-Maurice Delabar ${ }^{2}$; Michel Polak ${ }^{3}$

${ }^{1}$ INSERM U845, Université Paris Descartes, Hôpital Necker, 75015 Paris, France: ${ }^{2}$ University Paris Diderot-CNRS EAC 4413, Unit of Functional and Adaptative Biology (BFA), Paris, France; ${ }^{3}$ Paediatric endocrinology unit. APHP. Universite Paris Descartes, INSERM U845. Hôpital Necker de Paris, Paris, France

Background: Down syndrome (DS), also known as Trisomy 21, patients have a predisposition to congenital hypothyroidism, presumably because of thyroid dysgenesis (TD).

Objective and hypotheses: Our objectives are (1) to study if this predisposition to TD is caused by the overexpression of the DYRK1A gene, localized in the chromosome 21, which encodes the dual-specificity tyrosine-(Y)-phosphorylation regulated kinase 1A (Dyrk1A) and (2) to study if there is a link between Dyrk1A and the Shh signaling pathway.

Methods: We analyze the thyroid phenotype of transgenic mice expressing a third copy of this gene (TgDyrk1a mice), which are used as a model to study the DS.

Results: We show that the average size of thyroid follicles is smaller in TgDyrk1a adult thyroids compared to wild type (wt), suggesting an morphogenic default and/or a thyroid dysfunction. According to that, the blood thyroxin levels in TgDyrk1a mice are lower compared to wt. Shh signalling is known to be involved in thyroid organogenesis. Shh is a secreted protein which binds Ptc receptors, leading the Gli (Gli1, 2 and 3) factors then to regulate transcription of target genes. We show by in situ hybridization (ISH) that Ptc1 is expressed in wt thyroids from E9.5 to E15.5. In agreement with this, embryonic day 13.5 (E13.5) thyroids cultured in vitro are able to respond to Shh signalling. Shh and Gli2 are expressed in thyroids from E13.5 to E15.5. Gli3 is only detected in E13.5 thyroids, and its pattern of expression is complementary to the one of Gli2. Gli1 was not detected at any embryonic stage analyzed. We are currently studying the expression of the Shh pathway factors in TgDyrk1a embryonic thyroids.

Conclusions: We show for the first time that molecules involved in Shh signalling are expressed in thyroid anlagens in a narrow window during development. Our analysis will give new clues to understand the causes of TD predisposition in DS patients. This work is supported by FRM (DK), EDF (contrat RB 2010-12) (MP) and Sandoz SAS (MP).

\section{FC14-160 Thyroid}

\section{Low-normal FT4 and subclinical hypothyroidism may have a detrimental clinical effect in Down syndrome}

Eyal Wagner Lebel'; Ariel Tenenbaum ${ }^{1}$; Sarah Malkiel'; Yael Kastiel'; Abdulsalam Abu-Libdeh2; David Zangen ${ }^{2}$

${ }^{1}$ Hadassah Hebrew University Medical Center, Department of Pediatrics, Jerusalem, Israel; ${ }^{2}$ Hadassah Hebrew University Medical

Center, Division of Pediatric Endocrinology, Jerusalem, Israel

Background: The prevalence of hypothyroidism in Down syndrome is higher than in the normal population, reaching $15 \%$. Replacement therapy is crucial for preventing further compromise of intellectual and physical growth capacities. 
Objective and hypotheses: This study examined the relation between FT4 levels specifically within the normal range and clinical and developmental outcome in children with Down syndrome.

Methods: The study population consisted of 157 subjects who visited the Down Syndrome Medical Center at Hadassah Hospital between 2004-2008. These subjects' assessment included a medical history, physical and comprehensive neurological examination, developmental skills evaluation and blood tests for hemoglobin, FT4 and TSH.

Results: Hypothyroidism was found in $21.7 \%$ of the subjects, and subclinical hypothyroidism was detected in another $14.9 \%$. Moderate or severe hypotonia were more frequent among subjects with subclinical hypothyroidism compared to subjects without hypothyroidism (52.6\% Vs. $16.4 \%, \mathrm{P}=0.002)$. Upon comparison between subjects with normal FT4 levels below the median and subjects with FT4 levels above the median, hemoglobin levels were lower in subjects with FT4 levels below the median $(10.9 \%$ Vs. $0 \%$ below the normal range for age, $45.7 \%$ Vs. $23.8 \%$ below the mean, within the normal range for age, $\mathrm{P}=0.001$ ). Interestingly, among subjects with normal thyroid function tests, it was found that FT4 levels decrease with age (Pearson's correlation coefficient $=-0.324, \mathrm{P}=0.009$ ), but TSH levels do not change significantly. Conclusions: Subclinical hypothyroidism and low-normal FT4 levels, which are common in patients with Down syndrome, may have significant clinical sequelae, such as hypotonia and anemia. Preventive L-thyroxin replacement may be indicated specifically in the selected sub-populations of subclinical and low-normal FT4 cases. Our finding that FT4 levels decrease with age in Down syndrome (in contrast to the general population's trend) may indicate redefining of the normal range of laboratory values for FT4 in Down syndrome.

\section{FC14-161 Thyroid \\ Ten-years longitudinal study of thyroid function in children with Down syndrome}

Barbara Predieri'; Livia Garavelli'; Annalisa Bonetti'; Giulia Vellani'; Flavia Predieri'; Chiara Cattelani'; Simona Madeo'; Lorenzo lughetti ${ }^{1}$ University of Modena \& Reggio Emilia, Pediatrics, Modena, Italy; ${ }^{2}$ S. Maria Nuova Hospital, Pediatrics, Clinical Genetics Unit, Reggio Emilia, Italy

Background: Children with Down syndrome (DS) show an increased prevalence of thyroid disease when compared with the general population. The risk for thyroid dysfunction rises with age. A yearly thyroid screening was suggested in the guidelines of the American Academy of Pediatrics for the health supervision of children with DS.

Objective and hypotheses: The aim of our investigation is to longitudinally study the thyroid function in children with DS in order to identify the prevalence of thyroid pathology throughout development.

Methods: Thyroid function tests were yearly carried out on 120 children with DS, all followed from birth to 10 years of age. There were 65 boys and 55 girls. Subjects were annually classified according to thyroid function as: normal, congenital hypothyroidism (C-HT), hypothyroidism (HT - TSH $>10$ $\mathrm{mcIU} / \mathrm{ml}$ and low FT4), subclinical hypothyroidism (S-HT - TSH between 5 and $10 \mathrm{mcIU} / \mathrm{ml}$ and normal FT4), hyperthyroidism (HyperT).

Results: In our study population the prevalence of normal thyroid function significantly decreased from $90.8 \%$ to $41.7 \%$ throughout 10 -years follow-up. Therefore, the prevalence of thyroid dysfunctions increased, in particular the HT ones. The abnormal findings were almost equally distributed between the genders.

\begin{tabular}{lccccc}
$\begin{array}{l}\text { Follow- } \\
\text { up yearl } \\
\text { Prevalence } \\
\text { of thyroid } \\
\text { function }\end{array}$ & $\begin{array}{c}\text { Normal \% } \\
\text { (M/F) }\end{array}$ & $\begin{array}{c}\text { C-HT \% } \\
\text { (M/F) }\end{array}$ & HT \% (M/F) & S-HT \% (M/F) & $\begin{array}{c}\text { HyperT } \\
\%(M / F)\end{array}$ \\
1st & $90.8(58 / 51)$ & $9.2(7 / 4)$ & - & - & - \\
\hline 2nd & $71.7(44 / 42)$ & $9.2(7 / 4)$ & $2.5(2 / 1)$ & $16.6(12 / 8)$ & - \\
\hline 3rd & $65.8(41 / 38)$ & $9.2(7 / 4)$ & $7.5(5 / 4)$ & $17.5(12 / 9)$ & - \\
\hline 4th & $62.5(40 / 35)$ & $9.2(7 / 4)$ & $10.8(7 / 6)$ & $17.5(11 / 10)$ & - \\
\hline 5th & $60.0(37 / 35)$ & $9.2(7 / 4)$ & $11.6(8 / 6)$ & $19.2(13 / 10)$ & - \\
\hline 6th & $54.1(32 / 33)$ & $9.2(7 / 4)$ & $14.2(11 / 6)$ & $22.5(15 / 12)$ & - \\
\hline 7th & $49.2(28 / 31)$ & $9.2(7 / 4)$ & $15.8(12 / 7)$ & $25.8(18 / 13)$ & - \\
\hline 8th & $48.3(28 / 30)$ & $9.2(7 / 4)$ & $17.5(13 / 8)$ & $24.2(16 / 13)$ & $0.8(1 / 0)$ \\
\hline 9th & $44.2(26 / 27)$ & $9.2(7 / 4)$ & $18.3(14 / 8)$ & $27.5(17 / 16)$ & $0.8(1 / 0)$ \\
\hline 10th & $41.7(26 / 24)$ & $9.2(7 / 4)$ & $20.0(14 / 10)$ & $28.3(17 / 17)$ & $0.8(1 / 0)$ \\
\hline
\end{tabular}

Conclusions: More than half of the children with DS in our study developed thyroid dysfunctions. Our results confirm that DS patients, especially those with normal thyroid function and subclinical hypothyroidism, should be carefully followed annually to precociously identify the appearance of thyroid pathology and to begin an adequate hormonal treatment.

\section{FC14-162 Thyroid \\ Prevalence of thyroid disease in $22 q 11$ deletion syndrome: a retrospective chart review \\ Leona Fishman ${ }^{1}$; Jessica Shapiro²; Andrea L. Shugar'; \\ Jason Fishman ${ }^{3}$; Cheryl Cytrynbaum ${ }^{1}$; Rosanna Weksberg \\ ${ }^{1}$ Hospital for Sick Children, Clinical and Metabolic Genetics, Toronto, \\ Canada; ${ }^{2}$ University of Queensland, Medicine, Brisbane, Australia; \\ ${ }^{3}$ University of Western Ontario, Biomedical Science, London, Canada}

Background: 22q11.2 deletion syndrome (22q11 DS) is the most common gene deletion syndrome, occurring in 1/4000 individuals. The variable clinical presentation includes palatal anomalies, cardiac malformations, immune deficiency, hypoparathyroidism, developmental delay and psychiatric disorders. Our multidisciplinary clinic follows over 250 patients. Thyroid disease has been reported in this population, however no group has systematically estimate its prevalence. Understanding the relative risk for thyroid disease may assist in development of evidence-based guidelines for thyroid function monitoring.

Objective and hypothesis: i) estimate the prevalence of thyroid disease in our pediatric population and ii) characterize the thyroid disease phenotype in affected patients.

Hypothesis: Thyroid disease is more common in these patients as autoimmune disease more common.

Methods: Clinical data on 192 patients for a 5 year period: thyroid disease status, age at diagnosis, gender, parathyroid function status and cardiac lesion status. The data was analyzed to determine the prevalence of thyroid dysfunction, and to compare that prevalence to available general population data. Statistical tests were applied to investigate significant trends in gender differences, thyroid disease subtype (hyperthyroidism, hypothyroidism and sub-clinical disease) and co- morbid conditions.

Results: Of the 192 subjects, 99 male and 93 female. The average age 10.9 years. 18 patients $(9.4 \%)$ were thyroid disease positive; 3 (1.6\%) had hyperthyroidism, 15 (7.8\%) had hypothyroidism. The rate of disease was significantly higher than the general population, indicating a relative risk of 8.3 for hypothyroidism and 6.8 for hyperthyroidism in the 22q11 DS group. 23/192 (12\%)had subclinical disease, indicating a relative risk of 6.2; of these patients, 9 (39\%) later developed overt disease.

Conclusions: Based on these results, we recommend that clinicians be aware of the increased prevalence of thyroid disease in children with 22q11 DS. Affected individuals should undergo routine, lifelong thyroid function monitoring.

\section{FC14-163 Thyroid \\ The "customized" GCH-array \\ THYROARRAYv1.0® identifies genetic defects in congenital hypothyroidism not detectable by classical PCR and sequencing \\ Christian M. Moya ${ }^{1}$; Elena Vallespín²; Ariani Szkudlarek'; \\ Dulanjalee Kariyawasam ${ }^{3}$; Michel Polak'; Pablo Lapunzina2; Julián Nevado'; Jose Carlos Moreno' \\ IINGEMM- Institute for Medical and Molecular Genetics. "La Paz" University Hospital, Thyroid Molecular Laboratory, Madrid, Spain; 2INGEMM- Institute for Medical and Molecular Genetics. "La Paz" University Hospital, Structural and Functional Genomics Laboratory, Madrid, Spain; ${ }^{3}$ Necker-Enfants Malades Hospital, Pediatric Endocrinology, Paris, France}

Background: Currently 26 gene defects are known to cause congenital hypothyroidism $(\mathrm{CH})$. However, only a small percentage of patients harbour mutations which fully explain their thyroid phenotype. This could indicate the existence of other still unidentified thyroidal genes and/or that classical mutation screening by PCR and Sanger sequencing has technical limitations. Objective: To test the capacity of comparative genomic hybridization (CGH) arrays to identify deletions or duplications in $\mathrm{CH}$ patients without defects in the classical screening.

Methods: A "personalized” CGH-array was designed to study copy number 
alterations in thyroid-specific genes (TG, TPO, DUOX2, NIS, TSHR) and their transcription factors. An Agilent platform of 60000 oligos per genome was used. Average resolution in the areas of interest was 1 oligo per 150 bp and $125 \mathrm{Kbp}$ for the rest of the genome. Sixteen patients with thyroid dyshormonogenesis were studied not showing mutations in TPO, DUOX2 or DUOXA2 genes by PCR and sequencing.

Results: We identified heterozygous deletions of 1.2 and $10 \mathrm{Kbp}$ in the TG gene in two patients, excluding exons 20 and 45, respectively. In another patient, we identified a $50 \mathrm{bp}$ deletion which eliminates part of exon 16 of DUOX2, and also an intro-exonic duplication disrupting exon 5 of the same gene. In addition, one patient presented a 470 bp deletion that eliminated the codifying region of exon 12 in the PAX8 gene. Pathogenic predictions of these genetic aberrations are high and none of these are known as normal Copy Number Variations (CNVs).

Conclusions: A CGH-array with "customized" thyroid design is capable to detect genetic defects that escape the classical mutation analysis. A high density of oligos allows the identification of small deletions/duplications which even commercial arrays (Agilent 44K y 244K) would have not detected. Novel genomic techniques are essential in case of failed detection of mutations in the classical genes of human thyroid hormone synthesis.

\section{FC14-164 Thyroid}

Ten-year follow-up of a prospective cohort of children with Graves disease (GD), showing a positive impact of long-term antithyroid drug treatment on outcome

Juliane Léger'; Georges Gelwane'; Florentia Kaguelidou';

Meriem Benmerad; Corinne Alberti

${ }^{1}$ Hôpital Robert Debré, Paediatric Endocrinology Department, Centre de Référence Maladies Endocriniennes de la Croissance, Paris,

France; ' ${ }^{2}$ ôpital Robert Debré, Clinical Epidemiology Unit, INSERM

CIE5, Paris, France

Background: Controversy remains concerning the management of Graves hyperthyroidism in children, in the absence of an evidence-based optimal strategy for treating this condition. Most patients are initially treated with antithyroid drugs (ATD). However, fewer than $30 \%$ of children achieve lasting remission after about 2 years of ATD, so radioiodine therapy or near-total thyroidectomy is often proposed for definitive treatment.

Objective: The aim of this study was to assess the effect of the duration of carbimazole therapy on GD remission in children.

Methods: We conducted a prospective, multicenter cohort study of children with GD $(n=154)$. The intention was to treat patients with three consecutive courses of carbimazole, each lasting two years. The principal outcome measured was remission for at least 1.5 years after the completion of each course. The median duration of follow-up was $10.4(9.0$ - 12.1) years.

Results: Overall estimated remission rates $(95 \% \mathrm{CI})$ increased with time and were 20 (13-26), 37 (29-45), 45 (35-54) and 49 (40-57) \% after 4, 6, 8 and 10 years of follow-up, respectively. Multivariate competing risk models revealed a positive effect of less severe forms of hyperthyroidism at diagnosis [subhazard ratio (SHR) of 1 for patients with FT4 $<35 \mathrm{pmol} / 1$ vs $0.4(0.20$ 0.80 ) for FT $4 \geq 35 \mathrm{pmol} / \mathrm{l} ; \mathrm{p}=0.01]$ and of the presence of other autoimmune conditions [SHR of $2.27(1.2-4.3) ; \mathrm{p}=0.01$ ], on remission rate after medical treatment. By contrast to our previous report concerning the risk of relapse after the first course of treatment, no effect of ethnicity, age or serum TRAb levels at diagnosis was observed in the long term.

Conclusions: About half the patients achieved remission following carbimazole discontinuation, with a median follow-up of 10.4 years. We suggest that children with GD displaying good compliance with treatment should be offered long-term medical treatment with ATD before radical treatment is envisaged. 